\title{
On the Equations Defining Abelian Varieties. II
}

\section{Citation}

Mumford, David B. 1967. On the equations defining abelian varieties. II. Inventiones Mathematicae 3(2): 75-135.

\section{Published Version}

doi:10.1007/BF01389741

\section{Permanent link}

http://nrs.harvard.edu/urn-3:HUL.InstRepos:3597242

\section{Terms of Use}

This article was downloaded from Harvard University's DASH repository, and is made available under the terms and conditions applicable to Other Posted Material, as set forth at http:// nrs.harvard.edu/urn-3:HUL.InstRepos:dash.current.terms-of-use\#LAA

\section{Share Your Story}

The Harvard community has made this article openly available.

Please share how this access benefits you. Submit a story.

\section{Accessibility}




\title{
On the Equations Defining Abelian Varieties. II
}

\author{
D. Mumford (Cambridge, Mass.)
}

\section{Contents}

\$6. Structure of the Moduli Space . . . . . . . . . . . . . . . . . . . . 76

§. The 2-Adic Limit . . . . . . . . . . . . . . . . . . . . . . . . . 99

\$ 8. 2-Adic Theta Functions . . . . . . . . . . . . . . . . . . . . . . . 112

§. The 2-Adic Moduli Space . . . . . . . . . . . . . . . . . . . . . . 123

In the first part of this paper, we have analyzed a single abelian variety $X$. In particular, if $L$ is an ample invertible sheaf on $X$, we have analyzed the vector space $\Gamma(X, L)$ and the ring

$$
\oplus \Gamma\left(X, L^{n}\right)
$$

and have shown 1) how to choose canonical bases of these vector spaces, 2) how to express this ring as a quotient of a polynomial ring by an explicit homogeneous ideal involving coefficients which are essentially the "theta-null werte" of $X$. In this second part, we shall apply the first part to embed both the moduli spaces of abelian varieties, and the inverse limit of these spaces over successively higher levels, as open sets in projective schemes associated to homogeneous coordinate rings defined by explicit homogeneous ideals. We also introduce algebraic theta functions, defined on a 2-adic vector space in terms of which our results on moduli take on a simple form.

I want to offer some explanation of why the 2-adics play such a central role in this theory. The situation is this: if you stick to abelian varieties of char. $p(p \neq 2)$, then you can build up a theory of theta functions for these over any (restricted) product

$$
\prod_{l \in S}^{\prime} \boldsymbol{Q}_{l}
$$

where $S$ is any set of primes containing 2 , but not containing $p$. In other words, $\boldsymbol{Q}_{2}$ always has to be there, but you can throw in plenty of other factors if you like. Using only $Q_{2}$ seemed to have two advantages: (i) you can deal simultaneously with all characteristics except 2 , and (ii) the resulting theta-functions are more concise, i.e., are defined on the smallest locally compact group which admits them. I might have written a general theory for some arbitrary set $S$ - clearly this is the accepted French approach - but there seemed no point in not sticking to the simplest and most basic case. The essential features are related to the fact that multiplication by 2 does not preserve Haar measure.

6 Inventiones math., Vol. 3 


\section{§ 6. Structure of the Moduli Space}

To study questions of moduli, we must first have a theory of families of the objects to be classified. Therefore, we must generalize our theory to abelian schemes:

Definition. Let $S$ be a scheme. An abelian scheme $\mathscr{X}$ over $S$ is a group scheme $\mathscr{X}$ finitely presented over $S$ such that the projection $\pi: \mathscr{X} \rightarrow S$ is proper and smooth and the geometric fibres of $\pi$ are connected.

For some of the basic facts about abelian schemes, we refer the reader to [9], Ch. $6^{1}$.

Definition. Let $L$ be an invertible sheaf on $S$. Then 1. $H(L)$ is the group of sections $\alpha: S \rightarrow \mathscr{X}$ of $\pi$ such that if $T_{\alpha}: \mathscr{X} \rightarrow \mathscr{X}$ is translation by $\alpha$, then

$$
T_{\alpha}^{*} L \cong L \otimes \pi^{*} M
$$

for some invertible sheaf $M$ on $S$.

2. $H_{0}(L)$ is the subgroup of those $\alpha$ such that

$$
T_{\alpha}^{*} L \cong L .
$$

3. $\mathscr{G}(L)$ is the group of pairs $(\alpha, \varphi)$ where $\alpha \in H_{0}(L)$ and $\varphi: L \rightarrow T_{\alpha}^{*} L$ is an isomorphism.

Following a familiar procedure, we note first that $H$ and $\mathscr{G}$ can be extended to functors from the category of $S$-schemes to the category of groups:

Definition. For all $S$-schemes $f: T \rightarrow S$, let $H^{f}(L)=H\left(L^{\prime}\right), \mathscr{G}^{f}(L)=$ $\mathscr{G}\left(L^{\prime}\right)$, where if $F$ is the morphism in the diagram:

$$
\begin{aligned}
& \mathscr{X} \times{ }_{S} T \stackrel{F}{\longrightarrow} \mathscr{X} \\
& \downarrow \downarrow \\
& T \stackrel{f}{\longrightarrow} S
\end{aligned}
$$

then $L^{\prime}=F^{*} L$. Then $f \mapsto H^{f}(L), f \mapsto \mathscr{G}^{f}(L)$ are functors in an obvious way.

Proposition 1. Assume that $L$ is relatively ample over $S$. Then the 2 functors $f \mapsto H^{f}$ and $f \mapsto \mathscr{G}^{f}$ are representable by group schemes $\underline{H}(L)$, $\mathscr{G}(L)$ flat and of finite presentation over $S . \underline{H}(L)$ is a closed sub-group scheme of $\mathscr{X}$ itself, finite over $S$, and there is a canonical exact sequence:

$$
0 \rightarrow G_{m, s} \rightarrow \underline{G}(L) \rightarrow \underline{H}(L) \rightarrow 0 .
$$

\footnotetext{
${ }^{1}$ If any doubt should arise as to whether results in [9] are still valid if $S$ is nonnoetherian, it should be dispelled by noticing that any abelian scheme over an affine $S$ is obtained by base extension from an abelian scheme over $\operatorname{Spec}(R)$, where $R$ is a $Z$-algebra of finite type, [5], Ch. $4, \S 8,11$.
} 
Proof. First of all, $H(L)$ is nothing but the kernel of the canonical homomorphism: $\Lambda(L): \mathscr{X} \rightarrow \hat{\mathscr{X}} . \Lambda(L)$ is flat and finite and of finite presentation (cf. [9], p. 122), hence $\underline{H}(L)$ is flat and finite and finitely presented over $S$. Secondly, the morphism from the functor $\mathscr{G}^{f}$ to the functor $H^{f}$ is represented (relatively) by $G_{m}$-bundles. In fact, if $\alpha: T \rightarrow \mathscr{X} \times{ }_{s} T$ is an element of $H^{f}(T)$, and if

$$
M=\pi_{*}\left(T_{\alpha}^{*}\left(L^{\prime}\right) \otimes L^{\prime-1}\right)
$$

[here $L^{\prime}$ is the sheaf on $\mathscr{X} \times{ }_{S} T$ induced by $L$ and $\pi$ is the projection from $\mathscr{X} \times{ }_{S} T$ to $T$ ], then

$$
T_{\alpha}^{*} L^{\prime} \cong L^{\prime} \otimes \pi^{*} M,
$$

and multiplication by non-zero sections of $M$ defines the isomorphisms from $L^{\prime}$ to $T_{\alpha}^{*} L^{\prime}$. Therefore the relative functor in this case is represented by the line bundle $M$ on $T$ corresponding to $M^{-1}$, minus its 0 -section. Q.E.D.

Definition. Let $\delta=\left(d_{1}, d_{2}, \ldots, d_{g}\right)$ be any set of elementary divisors $\left(d_{i}\right.$ integers, $\left.\geqq 1, d_{i+1} \mid d_{i}\right)$. Define a functor $\mathscr{G}(\delta)$ on the category of all schemes $S$ to the category of groups by:

$$
\mathscr{G}^{S}(\delta)=\text { group of triples }(\alpha, x, l), \alpha \in \Gamma\left(S, 0_{S}^{*}\right),
$$

$x$ is a map from the set $\pi_{0}(X)$ of connected components of $X$ to $K(\delta)$, the discrete group $\oplus \boldsymbol{Z} / d_{i} \boldsymbol{Z}$.

$l=\left(l_{1}, \ldots, l_{g}\right)$, where $l_{i}$ is a $d_{i}$-th root of 1 in $\Gamma\left(S, 0_{S}^{*}\right)$.

Multiplication is:

where

$$
(\alpha, x, l) \cdot\left(\alpha^{\prime}, x^{\prime}, l^{\prime}\right)=\left(\alpha \cdot \alpha^{\prime} \cdot l^{\prime}(x), x+x^{\prime}, l+l^{\prime}\right)
$$

$$
\begin{aligned}
l^{\prime}(x), & \text { on the component } Y,=\prod_{i=1}^{\mathrm{g}} l_{i}^{a_{i}} \\
& \text { if } x(Y)=\left(a_{1}, \ldots, a_{g}\right) .
\end{aligned}
$$

[We add the $l$ 's instead of multiplying them to be consistent with our previous notation.]

It is easy to check that $S \mapsto \mathscr{G}^{S}(\delta)$ is represented by a group scheme $\mathscr{G}(\delta)$, flat and of finite type over $\boldsymbol{Z}$, that fits into a canonical exact sequence:

$$
0 \rightarrow G_{m} \rightarrow \underline{G}(\delta) \rightarrow \underline{H}(\delta) \rightarrow 0
$$

of group schemes, where

$$
\underline{H}(\delta)=\left[\underset{i}{\oplus} \boldsymbol{Z} / d_{i} Z\right] \oplus\left[\underset{i}{\oplus} \mu_{d_{i}}\right]
$$

and where $\boldsymbol{Z} / d_{i} \boldsymbol{Z}$ is taken as a discrete reduced group scheme and $\boldsymbol{\mu}_{d_{t}}$ is the usual group scheme of $d_{i}$-th roots of 1 .

6* 
Definition. A $\vartheta$-structure on a relatively ample invertible sheaf $L$ is an isomorphism over $S$ of the group schemes $\mathscr{G}(L)$ and $\mathscr{G}(\delta) \times S$, for some $\delta$, which is the identity on the sub-group schemes $\boldsymbol{G}_{m, s}$. When this exists, $\delta$ is called the type of $L$.

In this definition, we have included some types of non-separable invertible sheaves [in fact, all ample invertible sheaves on an abelian variety with $p^{g}$ points of order $p$ have a type in the above sense] because in the present categorical approach it is no trouble. However, this was just for the fun of it and we shall now restrict ourselves to the separable case.

Fix $\delta=\left(d_{1}, \ldots, d_{g}\right)$. Assume all $d_{i}$ even. Assume all schemes are schemes over Spec $Z\left[d^{-1}\right]$, where

$$
d=\prod_{i=1}^{\mathrm{g}} d_{i}
$$

Definition. An invertible sheaf $L$ on $\mathscr{X} / S$ is symmetric if $\imath^{*} L \cong L$, where $t: \mathscr{X} \rightarrow \mathscr{X}$ is the inverse. It is totally symmetric if there is an isomorphism $\varphi: L \widetilde{\sim}_{l} * L$ which restricts to the identity on $L \otimes \underline{O}_{\mathscr{X}_{2}}$, where $\mathscr{X}_{2} \subset \mathscr{X}$ is the kernel of $2 \delta$. It is normalized if $\varepsilon^{*} L \cong \underline{O}_{S}$ where $\varepsilon: S \rightarrow \mathscr{X}$ is the identity section.

Definition. Let $\lambda: \mathscr{G}(L) \sim \mathscr{G}(\delta) \times S$ be a $\vartheta$-structure for a symmetric relatively ample invertible sheaf $L$ on $\mathscr{X}$. Let $\psi: L \stackrel{\sim}{\rightarrow} \imath^{*} L$ be any isomorphism. We define:

i) an automorphism $\delta_{-1}$ of the functor $f \rightarrow \mathscr{G}^{f}(L)$. Given $f: T \rightarrow S$, let $L^{\prime}=L \otimes \underline{O}_{T}$ be the induced sheaf on $\mathscr{X} \times{ }_{S} T$. Let $\alpha: T \rightarrow \mathscr{X} \times{ }_{S} T$ be a section and

$$
\varphi: L^{\prime} \stackrel{\sim}{\longrightarrow} T_{\alpha}^{*}\left(L^{\prime}\right)
$$

an isomorphism, so that $(\alpha, \varphi) \in \mathscr{G}^{f}(L)$. Let $\psi^{\prime}: L^{\prime} \rightarrow \iota^{*} L^{\prime}$ be the isomorphism induced by $\psi$. Then

i.e.,

$$
\delta_{-1}((\alpha, \varphi))=\left(\imath \circ \alpha,\left(T_{\imath \circ \alpha}^{*} \psi^{\prime}\right)^{-1} \circ \imath^{*} \varphi \circ \psi^{\prime}\right),
$$

$$
\begin{aligned}
L^{\prime} \stackrel{\psi^{\prime}}{\sim} t^{*} & L^{\prime} \stackrel{\imath^{*} \varphi}{\sim} l^{*}\left(T_{\alpha}^{*} L^{\prime}\right) \\
& \left.T_{\imath \circ \alpha}^{*} \|^{*} L^{\prime}\right) \stackrel{\sim}{T_{1 \circ \alpha} \psi^{\prime}} T_{\imath \circ \alpha}^{*} L^{\prime},
\end{aligned}
$$

where $\imath: \mathscr{X} \times{ }_{s} T \rightarrow \mathscr{X} \times{ }_{s} T$ is the inverse.

ii) The automorphism $\delta_{-1}$ of the functor induces an automorphism $\underline{\delta}: \underline{G}(L) \rightarrow \underline{G}(L)$ of the scheme.

iii) Similarly, the map

$$
(\alpha, x, l) \mapsto(\alpha,-x,-l)
$$


where if $l=\left(l_{1}, \ldots, l_{g}\right)$, then $-l=\left(l_{1}^{-1}, \ldots, l_{g}^{-1}\right)$ gives an automorphism $D_{-1}$ of the functor $S \rightarrow \mathscr{G}^{S}(\delta)$.

iv) This induces an automorphism $D$ of the group scheme $\mathscr{G}(\delta)$.

v) Then $\lambda$ is symmetric if $\underline{D} \circ \lambda=\lambda \circ \underline{\delta}$.

Definition. We consider triples of the following type:

i) an abelian scheme $\mathscr{X}$ over $S$,

ii) a relatively ample, totally symmetric, normalized invertible sheaf $L$ on $\mathscr{X}$,

iii) a symmetric $\vartheta$-structure $\lambda: \mathscr{G}(L) \stackrel{\sim}{\rightarrow} \mathscr{G}(\delta) \times S$ for $L$.

We shall call this triple an abelian scheme with a $\delta$-marking.

Definition. For all schemes $S$, let $\mathscr{M}_{\delta}(S)$ denote the set of abelian schemes $\mathscr{X}$ over $S$ with $\delta$-markings, taken modulo isomorphisms. As $S$ varies, these sets form a functor $\mathscr{M}_{\delta}$ in $S$. This will be called the moduli functor for abelian schemes with $\delta$-marking.

The object of this section will be to show that $\mathscr{M}_{\delta}$ is representable, and to represent it by an open subset of a definite projective variety. The next step is to study the representations of $\mathscr{G}(\delta)$.

Definition. Let $V_{\delta}$ be the free $Z\left[d^{-1}\right]$-module of functions from $K(\delta)$ to $Z\left[d^{-1}\right]$. Then for all schemes $S$, and invertible sheaves $L$ on $S$, $V_{\delta} \otimes_{\mathbf{z}} L$ is the sheaf of functions from $K(\delta)$ to $L$. The discrete group $\mathscr{G}^{S}(\delta)$ acts $\underline{O}_{S}$-linearly on this sheaf, exactly as $\mathscr{G}(\delta)$ acted on $V_{\delta}$ in $\S 1$ : e.g., if $(\alpha, x, l) \in \mathscr{G}^{S}(\delta)$,

$$
f \in \Gamma\left(U, V_{\delta} \otimes_{\mathbf{z}} L\right) .
$$

Assume for simplicity that $U$ is connected, that $x(U)=\left(a_{1}, \ldots, a_{\mathrm{g}}\right)$ and that $l=\left(l_{1}, \ldots, l_{g}\right)$. Then $(\alpha, x, l)$ takes $f$ into $f^{*}$, where

$$
f^{*}\left(b_{1}, \ldots, b_{g}\right)=\alpha \cdot \prod l_{i}^{b_{i}} \cdot f\left(a_{1}+b_{1}, \ldots, a_{\mathrm{g}}+b_{\mathrm{g}}\right) .
$$

Let $\boldsymbol{E}_{\delta}=V\left(V_{\delta}\right)$ : this is a vector bundle of rank $d$ over Spec $Z\left[d^{-1}\right]$, and it is a direct sum of trivial line bundles $\boldsymbol{L}_{a}$ with canonical sections [a]: Recall that, in general, an $S$-valued point of $\boldsymbol{E}_{\delta}$ corresponds to a homomorphism from $V_{\delta}$ to $\Gamma\left(S, \underline{O}_{s}\right)$. Then $[a]$ corresponds to the homomorphism

$$
f \mapsto f(a)
$$

from $V(\delta)$ to $Z\left[d^{-1}\right]$. All the actions of $\mathscr{G}^{S}(\delta)$ on $V_{\delta} \otimes \underline{O}_{S}$ can be put together dually in an anti-representation of $\mathscr{G}(\delta)$ on $E_{\delta}$, i.e., a representation in which the order of multiplication is reversed. This is clear from 
a functorial point of view, but it may be useful to define this anti-representation directly by putting together representations on the various subgroups: on $E_{\delta}$,

the subgroup $G_{m}$, with $S$-valued points $(\alpha, 0,0)$, acts by homotheties

the discrete subgroup $\oplus \boldsymbol{Z} / d_{i} Z$, with $S$-valued points $(1, a, 0)$, acts by permuting the sections $[a]$ : thus the point $b$ takes $[a]$ to $[a+b]$,

the discrete but twisted subgroup $\oplus \boldsymbol{\mu}_{d_{i}}$ with $S$-valued points $(1,0, l)$, acts diagonally: thus the point $\left(l_{1}, \ldots, l_{g}\right)$ takes $[a]$ to $\Pi l_{i}^{a} \cdot[a]$.

Proposition 2. Let $S$ be a scheme and let

$$
\rho:[\underline{G}(\delta) \times S] \times{ }_{S} \boldsymbol{F} \rightarrow \boldsymbol{F}
$$

be an anti-representation over $S$ of $\mathscr{G}(\delta) \times S$ on a vector bundle $\boldsymbol{F}$ over $S$ of rank $d$. Assume that the subgroup $\boldsymbol{G}_{m}$ acts on $\boldsymbol{F}$ in the standard way. Then there is a line bundle Lover $S$ and an isomorphism

$$
\boldsymbol{F} \stackrel{\sim}{\longrightarrow}\left[\boldsymbol{E}_{\delta} \times S\right] \otimes \boldsymbol{L}
$$

(where $\otimes$ denotes tensor product of vector bundles over $S$ ) such that the action of $\mathscr{G}(\delta)$ on $\boldsymbol{F}$ corresponds to the above action of $\mathscr{G}(\delta)$ on $\boldsymbol{E}_{\delta}$ tensored with the trivial action on L. Moreover, this isomorphism is unique up to multiplication by an element of $\Gamma\left(S, \underline{O}_{S}^{*}\right)$.

Proof. This is nearly the same as that of Prop. $3, \S 1$, except that we must use a basic result on the representations of $\boldsymbol{\mu}_{d}$ established in [4]. It is shown there that if a group scheme of the form $\oplus \mu_{d_{i}}$ is represented, over $S$, in a vector bundle $\boldsymbol{F}$, then $\boldsymbol{F}$ is a direct sum of sub-vector bundles $F_{a}, a \in K(\delta)$, where the group acts on $\boldsymbol{F}_{a}$ by the character

$$
\begin{gathered}
\oplus \boldsymbol{\mu}_{d_{i}} \rightarrow \boldsymbol{G}_{\boldsymbol{m}} \\
\left(l_{1}, \ldots, l_{g}\right) \mapsto \prod_{i=1}^{\mathrm{g}} l_{i}^{a_{i}} .
\end{gathered}
$$

Now we realize $\oplus \mu_{d_{i}}$ as the subgroup of $\mathscr{G}(\delta)$ of triples $(1,0, l)$; and decompose $\boldsymbol{F}$ accordingly. Exactly as in $\S 1$, the action of the $Z\left[d^{-1}\right]$ valued points $\sigma_{a}=(1, a, 0)$ of $\mathscr{G}(\delta)$ permutes these $\boldsymbol{F}_{a}$ transitively. Therefore all $\boldsymbol{F}_{a}$ are non-empty; since the rank of $\boldsymbol{F}$ is $d$, each $\boldsymbol{F}_{a}$ is a line bundle over $S$. Let $\boldsymbol{L}=\boldsymbol{F}_{0}$. We set up the required isomorphism by first identifying

$$
F_{0}=L \cong\left(L_{0} \times S\right) \otimes L
$$

then identifying $\boldsymbol{F}_{a}$ with $\left(\boldsymbol{L}_{a} \times S\right) \otimes \boldsymbol{L}$ by using the actions of $\sigma_{a}$ and the first identification; and then taking the direct sum. The details work out exactly as in $\$ 1$. Q.E.D. 
Now start with an abelian scheme $\mathscr{X} / S$ with $\delta$-marking. Let $L$ be the given sheaf on $\mathscr{X}$, and let $\boldsymbol{F}=\boldsymbol{V}\left(\pi_{*} L\right)$ : this is a vector bundle over $S$ of rank $d$. Let $f: T \rightarrow S$ be a morphism of schemes. Then a $T$-valued point of $\boldsymbol{F} / S$, i.e., a morphism $g$ is the diagram:

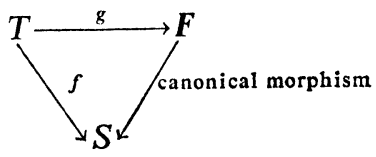

is the same thing as a homomorphism $\gamma$ :

$$
f^{*}\left(\pi_{*} L\right) \stackrel{\gamma}{\longrightarrow} \underline{O}_{T}
$$

of $\underline{O}_{T}$-modules. I claim that $\mathscr{G}(L)$ is anti-represented over $S$ on the vector bundle $F$ in a canonical way. In fact, if $f: T \rightarrow S$ is a morphism of schemes, a $T$-valued point of $\mathscr{G}(L) / S$ is given by a section

and an isomorphism

$$
\alpha: T \rightarrow \mathscr{X} \times{ }_{S} T
$$

$$
\varphi: L^{\prime} \stackrel{\sim}{\longrightarrow} T_{\alpha}^{*} L^{\prime}
$$

if $L^{\prime}$ is the induced sheaf on $\mathscr{X} \times{ }_{s} T$. Then $\varphi$ induces

$$
\begin{aligned}
& \pi_{*} L^{\prime} \stackrel{\varphi^{*}}{\longrightarrow} \pi_{*}\left(T_{\alpha}^{*} L^{\prime}\right) \\
& \text { l\| } \quad \text { l\| } \\
& f^{*}\left(\pi_{*} L\right) \quad\left(\pi \circ T_{\alpha}\right)_{*}\left(T_{\alpha}^{*} L^{\prime}\right) \\
& \text { l\| } \\
& \pi_{*}\left(T_{\alpha}\right)_{*} T_{\alpha}^{*}\left(L^{\prime}\right) \\
& \text { ? } \\
& \pi_{*} L^{\prime} \\
& f^{*}\left(\pi_{*} L\right)
\end{aligned}
$$

by standard canonical identifications. Let the composite isomorphism on $f^{*}\left(\pi_{*} L\right)$ be called $[\varphi]$. Then this acts on a $T$-valued point $\gamma: f^{*}\left(\pi_{*} L\right) \rightarrow \underline{O}_{T}$ of $\boldsymbol{F} / S$ by taking it to the new point $\gamma \circ[\varphi]$. This gives us an anti-representation of the functor $\mathscr{G}(L)$ on the functor associated to $F$ : hence an anti-representation of the scheme $\mathscr{G}(L)$ on $\boldsymbol{F}$.

But $\mathscr{X} / S$ has a given $\delta$-marking. Hence we also have an anti-representation over $S$ of $\mathscr{G}(\delta) \times S$ on $\boldsymbol{F}$. Applying Proposition 2, we get an isomorphism

$$
\boldsymbol{F} \stackrel{\sim}{\longrightarrow}\left(E_{\delta} \times S\right) \otimes L,
$$

for some line bundle $L$ on $S$, unique up to multiplication by elements of $\Gamma\left(S, O_{S}\right)$. In terms of sheaves, this gives us an isomorphism

$$
\pi_{*} L \stackrel{\sim}{\longleftarrow} V_{\delta} \otimes_{z} K
$$


for some invertible sheaf $K$ on $S$, unique up to multiplication by elements of $\Gamma\left(S, \underline{O}_{S}\right)$. The fact that the isomorphism of $\boldsymbol{F}$ and $\left(\boldsymbol{E}_{\delta} \times S\right) \otimes L \mathrm{com}$ mutes with pair of actions of $\mathscr{G}(L)$ and $\underline{G}(\delta)$ gives a corresponding fact on the sheaf level:

for all $f: T \rightarrow S$, let the $\vartheta$-structure induce an isomorphism

$$
\mathscr{G}\left(L^{\prime}\right)=\mathscr{G}^{f}(L) \cong \mathscr{G}^{T}(\delta)
$$

where $L^{\prime}$ is the induced sheaf on $\mathscr{X} \times{ }_{S} T$. Then the action of $\mathscr{G}\left(L^{\prime}\right)$ on $\pi_{*} L^{\prime}$ - which is canonically isomorphic to $f^{*} \pi_{*} L-$ goes over under this isomorphism to the action of $\mathscr{G}^{T}(\delta)$ on $V_{\delta} \otimes_{z} f^{*} K$.

It follows, incidentally, that the above isomorphism of $\pi_{*} L$ and $V_{\delta} \otimes_{z} K$ determines the isomorphism of $\mathscr{G}\left(L^{\prime}\right)$ and $\mathscr{G}^{T}(\delta)$ for all $f$ : hence it determines the given isomorphism of $\mathscr{G}(L)$ and $\mathscr{G}(\delta) \times S$. Now assume $L$ is relatively very ample: this occurs if all $d_{i}$ are divisible by 4 for example. Then we get a closed immersion over $S$ :

$$
i: \mathscr{X} \longrightarrow \boldsymbol{P}\left(\pi_{*} L\right) \cong \boldsymbol{P}\left[V_{\delta} \otimes_{\mathrm{z}} K\right] \cong \boldsymbol{P}\left(V_{\delta}\right) \times S,
$$

which is determined by the original $\delta$-marking on $\mathscr{X}$. Conversely, this immersion determines the $\delta$-marking: the sheaf $L$, for example, is obtained

a) by pulling back $\underline{O}_{\boldsymbol{P}}(1)$ via $p_{1} \circ i: \mathscr{X} \rightarrow \boldsymbol{P}\left(V_{\delta}\right)$,

b) by then normalizing this sheaf on the identity section. In other words

$$
L \cong\left(p_{1} \circ i\right)^{*}\left[\underline{O}_{P}(1)\right] \otimes\left(p_{1} \circ i \circ \varepsilon \circ \pi\right)^{*}\left[\underline{O}_{P}(-1)\right]
$$

And the morphism from $\mathscr{G}(\delta) \times S$ to $\mathscr{G}(L)$ is determined as follows: let $K=\left(p_{1} \circ i \circ \varepsilon\right)^{*}\left(\underline{O}_{P}(-1)\right)$. Then the isomorphism $\left(^{*}\right)$ determines the isomorphism:

$$
\begin{aligned}
\pi_{*} L \cong \pi_{*}\left[\left(p_{1} \circ i\right)^{*}\left(\underline{O}_{P}(1)\right)\right] \otimes K \\
\cong p_{2, *}\left[\underline{O}_{P}(1) \otimes \underline{O}_{S}\right] \otimes K \\
\cong V_{\delta} \otimes Z
\end{aligned}
$$

which, as we just saw, determines the $\vartheta$-structure. Summarizing, the abelian scheme $\mathscr{X} / S$ with $\delta$-marking determines, and is determined by the closed immersion:

$$
i: \mathscr{X} \longrightarrow \boldsymbol{P}\left(V_{\delta}\right) \times S \text {. }
$$

Recall that the group structure on $\mathscr{X}$ is determined completely by the identity section $\varepsilon: S \rightarrow \mathscr{X}$, ([9], p. 117). Therefore, the whole functor $\mathscr{M}_{\delta}$ is isomorphic to a subfunctor of the functor $\operatorname{Hilb}_{\boldsymbol{P}\left(V_{\delta}\right)}^{(1)}$ of all flat fa- 
milies of closed subschemes of $\boldsymbol{P}\left(V_{\delta}\right)$ with distinguished section (cf. [9], Ch. $0, \S 5)$.

In particular, we have defined, from $\mathscr{X} / S$ with $\delta$-marking, the $S$-valued point of $\boldsymbol{P}\left(V_{\delta}\right): p_{1} \circ i \circ \varepsilon: S \rightarrow \boldsymbol{P}\left(V_{\delta}\right)$. It is not hard to verify that this defines a morphism of functors:

$$
\mathscr{M}_{\delta} \stackrel{t}{\longrightarrow} h_{P\left(V_{\delta}\right)} \text {. }
$$

The next step is to describe the image. First define a closed subscheme $\bar{M}_{\delta} \subset \boldsymbol{P}\left(V_{\delta}\right)$ by RIEMANN's theta relations:

let $Q(a) \in V_{\delta}$ be the function which is 1 at $a$, and 0 elsewhere;

let $Z_{2} \subset K(\delta)$ be the subgroup of points of order 2; embed $K(\delta) \subset$ $K(2 \delta)$ as before.

1. For all elements $a, b, c, d \in K(2 \delta)$ such that they are all congruent modulo $K(\delta)$, and for all $l \in Z_{2}$, set

$$
\begin{aligned}
{\left[\sum_{\eta \in \mathbb{Z}_{2}} l(\eta) \cdot Q(a+\right.} & b+\eta) \cdot Q(a-b+\eta)] \times \\
\times & {\left[\sum_{\eta \in Z_{2}} l(\eta) \cdot Q(c+d+\eta) \cdot Q(c-d+\eta)\right]-} \\
- & {\left[\sum_{\eta \in Z_{2}} l(\eta) \cdot Q(a+d+\eta) \cdot Q(a-d+\eta)\right] \times } \\
\times & {\left[\sum_{\eta \in \mathbb{Z}_{2}} l(\eta) \cdot Q(c+b+\eta) \cdot Q(c-b+\eta)\right]=0 ; }
\end{aligned}
$$

2. For all $a \in K(\delta)$, set

$$
Q(a)-Q(-a)=0 .
$$

The main result can now be stated:

Theorem. If $d_{1}, \ldots, d_{\mathrm{g}}$ are all divisible by 8 , then there is an open subset $M_{\delta}$ of $\bar{M}_{\delta}$ such that $t$ defines an isomorphism of $\mathscr{M}_{\delta}$ with the functor of points of the subscheme $M_{\delta}$ of $\boldsymbol{P}\left(V_{\delta}\right)$.

We can go even further: not only can we identify the moduli scheme $M_{\delta}$, but we can write down the universal projective family of abelian varieties over $M_{\delta}$ obtained by the immersion $i$. Define

by the relations:

$$
\overline{A_{\delta}} \subset \boldsymbol{P}\left(V_{\delta}\right) \times \bar{M}_{\delta}
$$
factor:

let $X_{a}$ be the same function as $Q(a)$, but in the copy of $V_{\delta}$ in the first

1. For all $a, b, c, d, l$ as before, set

$$
\begin{aligned}
& {\left[\sum_{\eta \in Z_{2}} l(\eta) \cdot Q(c+d+\eta) \cdot Q(c-d+\eta)\right] \cdot\left[\sum_{\eta \in Z_{2}} l(\eta) \cdot X_{a+b+\eta} \cdot X_{a-b+\eta}\right]-} \\
& \quad-\left[\sum_{\eta \in Z_{2}} l(\eta) \cdot Q(c+b+\eta) \cdot Q(c-b+\eta)\right] \cdot\left[\sum_{\eta \in Z_{2}} l(\eta) \cdot X_{a+d+\eta} \cdot X_{a-d+\eta}\right]=0 .
\end{aligned}
$$


Thus via the projection $p_{2}, \bar{A}_{\delta}$ is a projective scheme over $\bar{M}_{\delta}$. Moreover, the diagonal $\Delta$ is a section

$$
\varepsilon: \bar{M}_{\delta} \rightarrow \bar{A}_{\delta}
$$

of the projective scheme $\bar{A}_{\delta} / \bar{M}_{\delta}$.

Further Theorem. Let $A_{\delta}=p_{1}^{-1}\left(M_{\delta}\right)$. Then $A_{\delta} / M_{\delta}$ with section $\Delta$ is an abelian scheme over $M_{\delta}$ and it is equal to the universal abelian scheme, with its identity section, embedded in $\boldsymbol{P}\left(V_{\delta}\right) \times M_{\delta}$ by the morphism i defined earlier in this section.

The remainder of this section will be devoted to proving these theorems.

Step 1. Without passing to any subset of $\bar{M}_{\delta}$ at all, $\bar{A}_{\delta} / \bar{M}_{\delta}$ has a kind of $\vartheta$-structure. In fact, let $M$ be the sheaf induced on $\bar{A}_{\delta}$ by $\underline{O}_{P}(1)$. Then for all morphisms

$$
f: T \rightarrow \bar{M}_{\delta},
$$

let $M^{\prime}$ be the induced sheaf on $\bar{A}_{\delta} \times \bar{M}_{\delta} T$. We get a canonical homomorphism:

$$
\{T \text {-valued points of } \underline{G}(\delta)\} \rightarrow\left\{\begin{array}{l}
\text { group of T-automorphisms } \\
\lambda: \bar{A}_{\delta} \times{ }_{M} T \rightarrow \bar{A}_{\delta} \times{ }_{M} T \\
\text { plus isomorphisms } \\
\varphi: M^{\prime} \stackrel{\sim}{\longrightarrow} \lambda^{*} M^{\prime}
\end{array}\right\} .
$$

And whenever $\bar{A}_{\delta} \times \bar{M}_{\delta} T$ is the subscheme of $\boldsymbol{P}\left(V_{\delta}\right) \times T$ induced from an abelian scheme with $\delta$-marking, these homomorphisms give us back the $\vartheta$-structure. To define this, assume for simplicity that $T$ is connected, and that

$$
\begin{gathered}
(\alpha, x, l) \in \mathscr{G}^{T}(\delta), \\
x=\left(a_{1}, \ldots, a_{g}\right), \\
l=\left(l_{1}, \ldots, l_{g}\right) .
\end{gathered}
$$

Then we get a projective transformation $\mu$ of $\boldsymbol{P}\left(V_{\delta}\right) \times T$ by the linear map

$$
\begin{gathered}
V_{\delta} \otimes \Gamma\left(T, \underline{O}_{T}\right) \rightarrow V_{\delta} \otimes \Gamma\left(T, \underline{O}_{T}\right), \\
f \mapsto f^{*}, \\
f^{*}\left(\left(b_{1}, \ldots, b_{g}\right)\right)=\alpha \cdot \prod l_{i}^{b_{i}} \cdot f\left(a_{1}+b_{1}, \ldots, a_{g}+b_{g}\right)
\end{gathered}
$$

(regarding $V_{\delta} \otimes \Gamma\left(T, \underline{O}_{T}\right)$ as $\Gamma\left(T, \underline{O}_{T}\right)$-valued functions on $K(\delta)$ ). In particular,

$$
X_{b} \otimes 1 \rightarrow X_{b-x} \otimes\left(\alpha \cdot \prod l_{i}^{b_{i}-a_{i}}\right) .
$$

One checks immediately that the equations defining $\bar{A}_{\delta}$ are invariant under this substitution. Therefore the projective transformation $\mu$ re- 
stricts to an automorphism $\lambda$ of $\bar{A}_{\delta} \times \bar{M}_{\delta} T$. But the linear map defining $\mu$ also defines an isomorphism

$$
\psi:\left[\underline{O}_{P}(1) \otimes \underline{O}_{T}\right] \stackrel{\sim}{\longrightarrow} \mu^{*}\left[\underline{O}_{P}(1) \otimes \underline{O}_{T}\right] .
$$

And $\psi$ restricts on $\bar{A}_{\delta} \times \bar{M}_{\delta} T$ to an isomorphism

$$
\varphi: M^{\prime} \stackrel{\sim}{\longrightarrow} \lambda^{*} M^{\prime} .
$$

Step II. In the first step, we have used a little bit the actual structure of the equations defining $\bar{M}_{\delta}$ : now we shall use this structure in detail. We want to define first a canonical morphism

which will be used in Step V.

$$
\bar{M}_{2 \delta} \stackrel{\pi}{\longrightarrow} \bar{M}_{\delta}
$$

Let $M\left(\right.$ resp. $\left.M^{\prime}\right)$ be the invertible sheaf on $\bar{M}_{\delta}$ (resp. $\bar{M}_{2 \delta}$ ) obtained by restricting $\underline{O}_{\boldsymbol{P}}(1)$ from the ambient $\boldsymbol{P}\left(V_{\delta}\right)$ (resp. $\left.\boldsymbol{P}\left(V_{2 \delta}\right)\right)$. By definition, we shall have

$$
\pi^{*}\left(M^{2}\right) \cong M^{\prime 2},
$$

and we define $\pi$ by its effect on the sections of the very ample sheaf $M^{2}$ :

$$
\pi^{*}[Q(a+b) \cdot Q(a-b)]=\sum_{\eta \in Z_{2}} Q^{\prime}(a+\eta) \cdot Q^{\prime}(b+\eta),
$$

where $Q$ and $Q^{\prime}$ are the sections of $M$ and $M^{\prime}$ defined above, and $a, b \in$ $K(2 \delta)$ satisfy $a+b \in K(\delta)$. To see that this defines a morphism $\pi$, we must check that when these values for $\pi^{*}$ are substituted, the following are zero:

$$
\begin{aligned}
& \pi^{*}[Q(a) \cdot Q(b)]-\pi^{*}[Q(b) \cdot Q(a)] \\
& \pi^{*}[Q(a) \cdot Q(b)] \cdot \pi^{*}[Q(c) \cdot Q(d)]- \\
& \quad-\pi^{*}[Q(a) \cdot Q(d)] \cdot \pi^{*}[Q(c) \cdot Q(b)] \\
& \pi^{*}[Q(-a) \cdot Q(b)]-\pi^{*}[Q(a) \cdot Q(b)]
\end{aligned}
$$$$
\text { for all } a, b, c, d \in K(\delta),
$$

$$
\begin{aligned}
\sum_{\eta \in Z_{2}} l(\eta) \cdot \pi^{*}[Q & (a+b+\eta) \cdot Q(a-b+\eta)] \times \\
& \times \sum_{\eta \in Z_{2}} l(\eta) \cdot \pi^{*}[Q(c+d+\eta) \cdot Q(c-d+\eta)]- \\
& -\sum_{\eta \in Z_{2}} l(\eta) \cdot \pi^{*}[Q(a+d+\eta) \cdot Q(a-d+\eta)] \times \\
& \times \sum_{\eta \in Z_{2}} l(\eta) \cdot \pi^{*}[Q(c+b+\eta) \cdot Q(c-b+\eta)] \\
& \text { for all } a, b, c, d \in K(2 \delta), l \in Z_{2} \text { such that } a, b, c, d \\
& \text { are congruent modulo } K(\delta) .
\end{aligned}
$$

The first 2 expressions in (1) are 0 by virtue of the relations imposed on the $Q^{\prime}$ 's; the last expression in (1) and the expression (2) are identically 
zero by virtue of the commutativity and associativity of multiplication in the $Q^{\prime}$ 's.

It is clear from the definition of $\pi$ that if $L$ is an invertible sheaf of type $\delta$ on an abelian variety and we choose symmetric $\vartheta$-structures on $\left(L, L^{2}\right)$, then the corresponding geometric points $x_{1}, x_{2}$ in $\bar{M}_{\delta}, \bar{M}_{2 \delta}$ are related by $\pi\left(x_{2}\right)=x_{1}$. The main result that we will need is:

Hardest Lemma. Let a geometric point $x$ in $\bar{M}_{2 \delta}$ be the theta-null point assigned to some abelian variety with $2 \delta$-marking. Then $\pi$ is étale in a neighborhood of $x$.

Proof. Let $y=\pi(x)$. By the infinitesimal criterion for a morphism to be étale ([3], $\S 3$, Cor. 3.2), we must check the following: let $A$ be an artin local ring with residue field $k$, the field of definition of $x$. Assume that $\sigma: \operatorname{Spec}(A) \rightarrow \bar{M}_{\delta}$ is an $A$-valued point extending $y: \operatorname{Spec}(k) \rightarrow \bar{M}_{\delta}$. Then there exists one and only one $A$-valued point $\tau: \operatorname{Spec}(A) \rightarrow \bar{M}_{2 \delta}$ such that $\sigma=\pi \circ \tau$, and such that $\tau$ extends $x$.

In down-to-earth language, suppose that at $x$, the coordinates $Q^{\prime}(a)$ have values $\bar{q}^{\prime}(a)$ in $k$; and that at $\sigma$, the coordinates $Q(a)$ have values $q(a)$ in $A$. Then $q(a), \bar{q}^{\prime}(a)$ satisfy the Riemann theta relation and symmetry, and if $\bar{q}(a)$ is the image of $q(a)$ in $k$, then $\bar{q}^{\prime}(a), \bar{q}(a)$ are related by (*). We must show that there are elements $q^{\prime}(a) \in A$ such that $q^{\prime}(a)$ lifts $\bar{q}^{\prime}(a)$, and is still a point of $\bar{M}_{2 \delta}$ and such that $q^{\prime}(a)$ and $q(a)$ are related by $(*)$. We have one more thing to help us: the values $\bar{q}(a)$ and $\bar{q}^{\prime}(a)$ come from an abelian variety.

The first thing to observe is that $(*)$ nearly determines $q^{\prime}(a)$ : in fact, we get

$$
\begin{aligned}
\sum_{\eta \in Z_{2}} l(\eta) \cdot q(a+b+\eta) \cdot q(a-b+\eta) & \\
& =\sum_{\eta \in Z_{2}} l(\eta) \cdot q^{\prime}(a+\eta) \cdot \sum_{\eta \in Z_{2}} l(\eta) \cdot q^{\prime}(b+\eta) .
\end{aligned}
$$

Since the $\bar{q}^{\prime}$ 's come from an abelian variety and since $4 \mid d_{i}$ for all $i$, we also have

For all $l \in Z_{2}$ and $a \in K(\delta)$, there is an element $b \in a+K(\delta)$ such that

$$
\sum_{\eta \in Z_{2}} l(\eta) \cdot \bar{q}^{\prime}(b+\eta) \neq 0
$$

(cf. §4, Proof of Theorem 1).

Set

$$
\begin{aligned}
U(a, b, l) & =\sum_{\eta \in Z_{2}} l(\eta) \cdot q(a+b+\eta) \cdot q(a-b+\eta), \\
x(a, l) & =\sum_{\eta \in Z_{2}} l(\eta) \cdot q^{\prime}(a+\eta), \\
\bar{x}(a, l) & =\sum_{\eta \in Z_{2}} l(\eta) \cdot \bar{q}^{\prime}(a+\eta) .
\end{aligned}
$$


For each $l \in Z_{2}$, and $\alpha \in K(2 \delta) / K(\delta)$, choose an element $a_{0} \in K(2 \delta)$ lifting $\alpha$ such that $\bar{x}\left(a_{0}, l\right) \neq 0$. Then $U\left(a_{0}, a_{0}, l\right)$ is a unit in $A$, and $x\left(a_{0}, l\right)$ is to satisfy:

i) $x\left(a_{0}, l\right)^{2}=U\left(a_{0}, a_{0}, l\right), x\left(a_{0}, l\right) \mapsto \bar{x}\left(a_{0}, l\right)$ in $k$.

Since $\operatorname{char}(k) \neq 2$, this determines one and only one $x\left(a_{0}, l\right)$. For any other $a \in K(2 \delta)$ lifting $\alpha$, set

ii) $x(a, l)=\frac{U\left(a, a_{0}, l\right)}{x\left(a_{0}, l\right)}$.

From the $x$ 's, we determine the $q^{\prime}$ 's by summing over $l$. This proves the uniqueness of the values $q^{\prime}(a)$, i.e., of the point $\tau$, and shows that $\pi$ is unramified at $x$. It remains to show that if $\{x(a, l)\}$, hence $\left\{q^{\prime}(a)\right\}$ are determined by i), ii), then they satisfy all the requirements.

First of all, $x(a, l)$ lifts $\bar{x}(a, l)$ since $\bar{x}(a, l)$ satisfies (ii) with bars in it. Hence $q^{\prime}(a)$ lifts $\bar{q}^{\prime}(a)$.

Secondly, $x(a, l)$ and $U(a, b, l)$ satisfy $(*)^{\prime}:$ let $a, b \in K(2 \delta)$ both lie over $\alpha$. Then

$$
\begin{aligned}
x(a, l) \cdot x(b, l) & =\frac{U\left(a, a_{0}, l\right) \cdot U\left(b, a_{0}, l\right)}{x\left(a_{0}, l\right)^{2}} \\
& =\frac{U\left(a, a_{0}, l\right) \cdot U\left(b, a_{0}, l\right)}{U\left(a_{0}, a_{0}, l\right)} \\
& =U(a, b, l)
\end{aligned}
$$

by RIEMANN's theta relation for the $q$ 's. Therefore, the corresponding $q^{\prime}(a)$ 's satisfy $(*)$.

Thirdly, $q^{\prime}$ is even. In fact, $I$ claim $x(-a, l)=x(a, l)$. This follows from the equation $U\left(-a, a_{0}, l\right)=U\left(a, a_{0}, l\right)$ which follows from the evenness of $q$.

Fourthly, we must check that $q^{\prime}$ satisfies RIEMANN's theta relation. This is really rather tricky. It is convenient to use a different form of these relations:

let $H=K(2 \delta) \times \hat{Z}_{4}$

$$
\left[\text { n.b.: } \hat{Z}_{4} \text { is the discrete group } \operatorname{Hom}\left(Z_{4}, A^{*}\right)\right] \text {. }
$$

If $b_{i}=\left(a_{i}, l_{i}\right) \in H, i=1,2$, then set

$$
\begin{aligned}
C\left(b_{1}, b_{2}\right) & =\sum_{\substack{\eta_{1}, \eta_{2} \in Z_{4} \\
\eta_{1}+\eta_{2} \in Z_{2}}} l_{1}\left(\eta_{1}\right) l_{2}\left(\eta_{2}\right) q^{\prime}\left(a_{1}+\eta_{1}\right) \cdot q^{\prime}\left(a_{2}+\eta_{2}\right) \\
& =\frac{1}{2^{g}} \sum_{\eta \in Z_{4}}\left(l_{1}+l_{2}\right)(\eta) \cdot x\left(a_{1}+\eta, \bar{l}_{1}\right) \cdot x\left(a_{2}+\eta, \bar{l}_{2}\right)
\end{aligned}
$$

[here $\bar{l}_{i}=$ restrictions of $l_{i}$ to $Z_{2}$ ]. 
The relations are:

$$
C\left(b_{1}, b_{2}\right) \cdot C\left(b_{3}, b_{4}\right)=C\left(b_{1}+\beta, b_{2}+\beta\right) \cdot C\left(b_{3}+\beta, b_{4}+\beta\right)
$$

$$
\text { for all } b_{1}, b_{2}, b_{3}, b_{4}, \beta \in H \text { such that }
$$

$$
b_{1}+b_{2}+b_{3}+b_{4}=-2 \beta \text {. }
$$

To check that these relations are equivalent to the ones we have been using involving $U^{\prime}$ 's is quite straightforward and we omit it. [The method is the same as the one we will use in the next step.]

We also need a third form of these relations, that is, the Riemann form itself involving the $x$ 's (cf. $\S 3$, right at the end):

$$
\begin{aligned}
x\left(a_{1}, l_{1}\right) \cdot x\left(a_{2}, l_{2}\right) \cdot x\left(a_{3}, l_{3}\right) \cdot x\left(a_{4}, l_{4}\right) & \\
= & \frac{1}{2^{28}} \sum_{\substack{k \in \hat{Z}_{2} \\
\eta \in Z_{4}}} k(2 \eta) \cdot x\left(a_{1}+b+\eta, l_{1}+k\right) \cdot x\left(a_{2}+b+\eta, l_{2}+k\right) \times \\
& \times x\left(a_{3}+b+\eta, l_{3}+k\right) \cdot x\left(a_{4}+b+\eta, l_{4}+k\right)
\end{aligned}
$$

$$
\text { for all } a_{1}, a_{2}, a_{3}, a_{4}, b \in K(2 \delta)
$$

$$
l_{1}, l_{2}, l_{3}, l_{4} \in \ddot{Z}_{2}
$$

such that

$$
\begin{aligned}
& a_{1}+a_{2}+a_{2}+a_{4}=-2 b, \\
& l_{1}+l_{2}+l_{3}+l_{4}=0 .
\end{aligned}
$$

This is how to go back and forth between $(* *)$ and $(* * *)$ :

1) take expression (**), substitute $l_{1}+2 k_{1}$ for $l_{1}, l_{3}+2 k_{3}$ for $l_{3}$, and $m-k_{1}-k_{3}$ for $m$, [here $b_{i}=\left(a_{i}, l_{i}\right)$, and $\beta=(\alpha, m)$ ]. Sum over all choices of $k_{1}, k_{3}$, rearrange the right-hand side and you get $(* * *)$.

2) take expression (***), substitute $a_{1}+\zeta$ for $a_{1}, a_{2}+\zeta$ for $a_{2}, a_{3}+\zeta^{\prime}$ for $a_{3}, a_{4}+\zeta^{\prime}$ for $a_{4}, b-\zeta-\zeta^{\prime}$ for $b$, multiply by $\left(l_{1}+l_{2}\right)(\zeta) \cdot\left(l_{3}+l_{4}\right)\left(\zeta^{\prime}\right)$, and sum over all $\zeta, \zeta^{\prime} \in Z_{4}$. Rearrange the right-hand side and you get $(* *)$.

The point to notice here is that you don't need all of the relations $(* *)$ to get a particular one of the relations $(* * *)$, or vice versa.

Next, there are some relations $(* *)$ that we do get immediately. In fact, suppose $b_{1}+b_{2} \in 2 H$. Then $a_{1}+a_{2} \in K(\delta)$ and $l_{1}=l_{2}$ on $\hat{Z}_{2}$. Using $(*)^{\prime}$, one finds that $C\left(b_{1}, b_{2}\right)$ splits into a product:

$$
\begin{aligned}
C\left(b_{1}, b_{2}\right)= & \frac{1}{2^{2 g}}\left[\sum_{\zeta \in Z_{4}}\left(l_{1}+l_{2}\right)(\zeta) \cdot q\left(a_{1}+a_{2}+2 \zeta\right)\right] \times \\
& \times\left[\sum_{\zeta \in Z_{4}}\left(l_{1}-l_{2}\right)(\zeta) \cdot q\left(a_{1}-a_{2}+2 \zeta\right)\right] .
\end{aligned}
$$


One gets similar equations for $C\left(b_{3}, b_{4}\right), C\left(b_{1}+\beta, b_{2}+\beta\right)$ and $C\left(b_{3}+\beta\right.$, $\left.b_{4}+\beta\right)$ : substituting, one gets $(* *)$ from the associative law.

Next, we can show that the relations (**) also hold if $b_{1}+b_{3} \in 2 H$. We prove this in 3 steps using the precise description of which relations $(* *)$ are needed to prove which relations $(* * *)$ and vice versa.

i) relations (**) for all values of $b_{1}, b_{2}, b_{3}, b_{4}, \beta$ such that $b_{1}+b_{2} \in 2 H$ imply relations (***) for all $a_{i}, l_{i}, b$ such that $a_{1}+a_{2} \in K(\delta), l_{1}=l_{2}$ on $Z_{2}$.

ii) relations $(* * *)$ are symmetric under permutation of the variables.

So we also get relations $(* * *)$ when $a_{1}+a_{3} \in K(\delta), l_{1}=l_{3}$ on $Z_{2}$.

iii) Using the fact that $Z_{4} \subset K(\delta)$, if $a_{1}+a_{3} \in K(\delta)$, then $a_{1}+a_{3}+\zeta+\zeta^{\prime} \in$ $K(\delta)$ and we can go back: we get relations (**) whenever $b_{1}+b_{3} \in 2 H$.

Next, we can show that the square of relation $(* *)$ is always true:

$$
C\left(b_{1}, b_{2}\right)^{2} \cdot C\left(b_{3}, b_{4}\right)^{2}=C\left(b_{1}+\beta, b_{2}+\beta\right)^{2} \cdot C\left(b_{3}+\beta, b_{4}+\beta\right)^{2} .
$$

In fact, for all $b_{1}, b_{2} \in H$,

$$
\begin{aligned}
C\left(b_{1}, b_{2}\right)^{2} & =C\left(b_{1}, b_{2}\right) \cdot C\left(b_{1},-b_{2}\right) \\
& =C\left(0, b_{2}-b_{1}\right) \cdot C\left(0,-b_{1}-b_{2}\right)
\end{aligned}
$$

by relation $(* *)$ with $\beta=-b_{1}$

Therefore:

$$
=C\left(b_{1}-b_{2}, 0\right) \cdot C\left(b_{1}+b_{2}, 0\right) \text {. }
$$

$C\left(b_{1}+\beta, b_{2}+\beta\right)^{2} \cdot C\left(b_{3}+\beta, b_{4}+\beta\right)^{2}$

$$
\begin{aligned}
= & C\left(b_{1}+b_{2}+2 \beta, 0\right) \cdot C\left(b_{1}-b_{2}, 0\right) \cdot C\left(b_{3}+b_{4}+2 \beta, 0\right) \times \\
& \times C\left(b_{3}-b_{4}, 0\right) .
\end{aligned}
$$

But

$$
\begin{aligned}
b_{1}+b_{2}+2 \beta & =-b_{3}-b_{4}, \text { and } b_{3}+b_{4}+2 \beta=-b_{1}-b_{2}: \text { so we get } \\
& =C\left(b_{1}+b_{2}, 0\right) \cdot C\left(b_{1}-b_{2}, 0\right) \cdot C\left(b_{3}+b_{4}, 0\right) \cdot C\left(b_{3}-b_{4}, 0\right) \\
& =C\left(b_{1}, b_{2}\right)^{2} \cdot C\left(b_{3}, b_{4}\right)^{2} .
\end{aligned}
$$

But the Riemann relations definitely hold when you take the image of $C\left(b_{1}, b_{2}\right)$, etc., in $k$. Therefore, if

$$
\bar{C}\left(b_{1}, b_{2}\right) \cdot \bar{C}\left(b_{3}, b_{4}\right) \neq 0 \quad \text { in } k,
$$

it follows that $C\left(b_{1}, b_{2}\right) \cdot C\left(b_{3}, b_{4}\right)$ and $C\left(b_{1}+\beta, b_{2}+\beta\right) \cdot C\left(b_{3}+\beta\right.$, $\left.b_{4}+\beta\right)$ are square roots of the same number, are units, and have the same images in $k$ : hence they are equal. 
Now suppose

$$
\begin{aligned}
& b_{1}+b_{2}+b_{3}+b_{4}=-2 \beta \\
& b_{1}^{\prime}+b_{2}^{\prime}+b_{3}^{\prime}+b_{4}^{\prime}=-2 \beta^{\prime}
\end{aligned}
$$

where $b_{i}+b_{i}^{\prime} \in 2 H$, all $i$, and $\beta+\beta^{\prime} \in 2 H$. Assume moreover that

$$
C\left(b_{1}^{\prime}, b_{2}^{\prime}\right) \cdot C\left(b_{3}^{\prime}, b_{4}^{\prime}\right) \quad \text { is a unit. }
$$

Then we can also show that

$$
C\left(b_{1}, b_{2}\right) \cdot C\left(b_{3}, b_{4}\right)=C\left(b_{1}+\beta, b_{2}+\beta\right) \cdot C\left(b_{3}+\beta, b_{4}+\beta\right) .
$$

In fact, we know this equality, when there are primes everywhere. Hence it suffices to show:

$$
\begin{aligned}
C\left(b_{1}, b_{2}\right) \cdot C\left(b_{1}^{\prime}, b_{2}^{\prime}\right) \cdot C\left(b_{3}, b_{4}\right) \cdot C\left(b_{3}^{\prime}, b_{4}^{\prime}\right) \\
=C\left(b_{1}+\beta, b_{2}+\beta\right) \cdot C\left(b_{1}^{\prime}+\beta^{\prime}, b_{2}^{\prime}+\beta^{\prime}\right) \cdot C\left(b_{3}+\beta, b_{4}+\beta\right) \times \\
\quad \times C\left(b_{3}^{\prime}+\beta^{\prime}, b_{4}^{\prime}+\beta^{\prime}\right) .
\end{aligned}
$$$$
(\alpha)
$$

But assume $b_{i}+b_{i}^{\prime}=-2 \gamma_{i}$. Then using the relations $(* *)$ that we know to be true, we get:

$$
\begin{aligned}
C\left(b_{1}, b_{2}\right) \cdot C\left(b_{1}^{\prime},-b_{2}^{\prime}\right) & \\
= & C\left(\gamma_{1}-\gamma_{2}+b_{1}-b_{2}, \gamma_{1}-\gamma_{2}\right) \cdot C\left(\gamma_{1}+\gamma_{2}+b_{1}^{\prime}+b_{2}^{\prime}, \gamma_{1}+\gamma_{2}\right) \\
C\left(b_{3}, b_{4}\right) \cdot C\left(b_{3}^{\prime},-b_{4}^{\prime}\right) & \\
= & C\left(\gamma_{3}-\gamma_{4}+b_{3}-b_{4}, \gamma_{3}-\gamma_{4}\right) \cdot C\left(\gamma_{3}+\gamma_{4}+b_{3}^{\prime}+b_{4}^{\prime}, \gamma_{3}+\gamma_{4}\right) \\
C\left(b_{1}+\right. & \left.\beta, b_{2}+\beta\right) \cdot C\left(b_{1}^{\prime}+\beta^{\prime},-b_{2}^{\prime}-\beta^{\prime}\right) \\
= & C\left(b_{1}-b_{2}+\gamma_{1}-\gamma_{2}, \gamma_{1}-\gamma_{2}\right) \times \\
& \times C\left(\gamma_{1}+\gamma_{2}+b_{1}^{\prime}+b_{2}^{\prime}+\beta^{\prime}-\beta, \gamma_{1}+\gamma_{2}-\beta-\beta^{\prime}\right) \\
C\left(b_{3}+\right. & \left.\beta, b_{4}+\beta\right) \cdot C\left(b_{3}^{\prime}+\beta^{\prime},-b_{4}^{\prime}-\beta^{\prime}\right) \\
= & C\left(b_{3}-b_{4}+\gamma_{3}-\gamma_{4}, \gamma_{3}-\gamma_{4}\right) \times \\
& \times C\left(\gamma_{3}+\gamma_{4}+b_{3}^{\prime}+b_{4}^{\prime}+\beta^{\prime}-\beta, \gamma_{3}+\gamma_{4}-\beta-\beta^{\prime}\right) .
\end{aligned}
$$

We can assume that $\gamma_{1}+\gamma_{2}+\gamma_{3}+\gamma_{4}=\beta+\beta^{\prime}$ by altering one of the $\gamma^{\prime}$ 's by an element of order 2 . Using the symmetries $C(A, B)=C(A,-B)=$ $C(B, A)$ one checks that the product of the 1 st two right-hand sides equals the product of the 2 nd two. Hence the same for the left-hand sides, hence $(\alpha)$ is true. 
It remains only to check that for any $b_{1}, b_{2}, b_{3}, b_{4}, \beta$ such that $b_{1}+b_{2}+b_{3}+b_{4}=-2 \beta$, there exists $b_{1}^{\prime}, b_{2}^{\prime}, b_{3}^{\prime}, b_{4}^{\prime}, \beta^{\prime}$ differing from the $b_{i}$ 's and $\beta$ by $2 H$, such that

$$
\begin{aligned}
& b_{1}^{\prime}+b_{2}^{\prime}+b_{3}^{\prime}+b_{4}^{\prime}=-2 \beta^{\prime} \\
& \bar{C}\left(b_{1}^{\prime}, b_{2}^{\prime}\right) \cdot \bar{C}\left(b_{3}^{\prime}, b_{4}^{\prime}\right) \neq 0 .
\end{aligned}
$$

Now we know that the functions $\bar{q}, \bar{q}^{\prime}$ come from an abelian variety. Therefore choosing a compatible $4 \delta$-marking on this abelian variety, there is a null-value function $\bar{q}^{\prime \prime}$ on $K(4 \delta)$ with values in $k$, such that

Now if $a \in K(4 \delta), l \in \hat{Z}_{4}$, set:

$$
\bar{q}^{\prime}(a+b) \cdot \bar{q}^{\prime}(a-b)=\sum_{\eta \in Z_{2}} \bar{q}^{\prime \prime}(a+\eta) \cdot \bar{q}^{\prime \prime}(b+\eta) .
$$

$$
\bar{z}(a, l)=\sum_{\eta \in \mathbf{Z}_{4}} l(\eta) \bar{q}^{\prime \prime}(a+\eta) .
$$

Then if $b_{1}=(a+b, l), b_{2}=(a-b, m)$ are 2 elements of $H$, with $a, b \in K(4 \delta)$, $l, m \in \hat{Z}_{4}$, one checks immediately that:

$$
\bar{C}\left(b_{1}, b_{2}\right)=\bar{z}(a, l+m) \cdot \bar{z}(b, l-m) .
$$

Therefore, translating the question into one involving $\bar{z}$ 's, we have to check:

$$
\begin{cases}\text { for all } a_{1}, a_{2}, a_{3}, a_{4} \in K(4 \delta), & \text { congruent } \bmod K(2 \delta), \\ \text { for all } l_{1}, l_{2}, l_{3}, l_{4} \in \hat{Z}_{4}, & \text { congruent } \bmod 2 \hat{Z}_{4}, \\ \text { there exists } \alpha_{1}, \alpha_{2}, \alpha_{3}, \alpha_{4} \in K(2 \delta), & \text { congruent } \bmod K(\delta) \\ \text { and } k \in 2 \hat{Z}_{4}, \text { such that } & 4 \\ \prod_{i=1}^{4} \bar{z}\left(a_{i}+\alpha_{i}, l_{i}+m\right) \neq 0 .\end{cases}
$$

But using the hypothesis that $Z_{8} \subset K(\delta)$, this follows from:

$$
\left\{\begin{aligned}
\text { for all } & a \in K(4 \delta), l \in \hat{Z}_{4}, \text { there exists } \alpha \in Z_{8} \text { such that } \\
& \bar{z}(a+\alpha, l) \neq 0 .
\end{aligned}\right.
$$

This is a special case of the result stated following Theorem $1, \S 4$. Phew!

The same analysis also gives the result:

If $\left\{\bar{q}^{\prime}(a)_{1}\right\}$ are the coordinates of a geometric point $x_{1}$ in $\bar{M}_{2 \delta}$ which corresponds to an abelian variety with $2 \delta$-marking, then the coordinates $\left\{\bar{q}^{\prime}(a)_{2}\right\}$ of any other geometric point $x_{2}$ such that $\pi\left(x_{1}\right)=\pi\left(x_{2}\right)$ are determined by:

$$
\sum_{\eta \in Z_{2}} l(\eta) \bar{q}^{\prime}(a+\eta)_{2}=\gamma(a, l) \cdot \sum_{\eta \in Z_{2}} l(\eta) \cdot \bar{q}^{\prime}(a+\eta)_{1}
$$

7 Inventiones math., Vol. 3 
where $\gamma$ is a quadratic character:

$$
\gamma: K(2 \delta) / K(\delta) \times Z_{2} \rightarrow\{ \pm 1\} .
$$

We leave it to the reader to check this change from the $\bar{q}^{\prime}(a)_{1}$ 's to the $\bar{q}^{\prime}(a)_{2}$ 's also comes about by a suitable modification of the $2 \delta$-marking. Therefore:

if a geometric point $x$ of $\bar{M}_{2 \delta}$ corresponds to an abelian variety with $2 \delta$-marking, so do all geometric points in $\pi^{-1}(\pi(x))$.

Step III. Let $\mathscr{X} / S$ be an abelian scheme with $\delta$-markings. Let $i: \mathscr{X} \hookrightarrow \boldsymbol{P}\left(V_{\delta}\right) \times S$ be the closed immersion defined above. Then

i) $p_{1} \circ i \circ \varepsilon$ is actually a morphism $j$ of $S$ to $\bar{M}_{\delta}$,

ii) $i(\mathscr{X})$ equals the subscheme $y$ of $\boldsymbol{P}\left(V_{\delta}\right) \times S$ obtained as the fibre product:

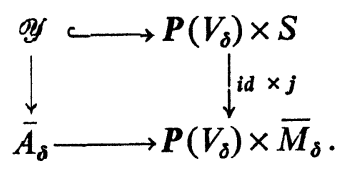

In order to establish this, it clearly suffices to take the case $S=\operatorname{Spec}(A)$, where $A$ is an artin local ring with algebraically closed residue field. Let $p$ be the characteristic of the residue field $k$. Then there are considerable technical simplifications:

a) for all positive integers $n$ not divisible by $p, A$ contains exactly $n n$-th roots of 1 , which lift the $n n$-th roots of 1 in $k$,

b) if $\mathscr{X} / S$ is an abelian scheme, then for all positive integers $n$ not divisible by $p$, the subscheme $\mathscr{X}_{n}$ of points of order $n$ is the disjoint union of $n^{2 g}$ subschemes, each being the image of a section of $\mathscr{X} / S$ : i.e., all points of order $n$ are rational over $S$.

Cor. of $(a),(b)$. Let $\mathscr{X} / S$ be an abelian scheme, and let $X / k$ be its fibre over the residue field. Let $L$ be a relatively ample sheaf of degree $d$ on $\mathscr{X}$. Then $H(L)$ is a disjoint union of the images of $d^{2}$ distinct sections of $\mathscr{X} / S$, and there is a one-one correspondence between $\vartheta$-structures for $L$ and for the induced sheaf $\bar{L}$ on $X$.

In this case, we can deal entirely with the group of rational points $\mathscr{G}^{S}(\delta)$ instead of with the combersome group schemes. Thus $\mathscr{G}^{S}(\delta)$ is just the (discrete) group extension:

$$
0 \rightarrow A^{*} \rightarrow \mathscr{G}^{S}(\delta) \rightarrow K(\delta) \times \widehat{K(\delta)} \rightarrow 0
$$

and where $\widehat{K(\delta)}$ is just a (discrete) group isomorphic to $K(\delta)$. And the representation theory boils down to: 
There is a unique $A$-linear representation of $\mathscr{G}^{S}(\delta)$ on a free $A$-module of rank $d$, in which the subgroup $A^{*}$ acts by homotheties.

In particular, a $\vartheta$-structure on $L$ induces an isomorphism:

$$
\Gamma(\mathscr{X}, L) \cong V_{\delta} \otimes_{\mathrm{z}} A
$$

unique up to multiplication by elements of $A^{*}$.

To get down to the proof itself, we are given $\mathscr{X} / S$, plus a totally symmetric relatively ample $L$, plus a symmetric isomorphism $f_{1}$ of $\mathscr{G}(L)$ and $\mathscr{G}^{S}(\delta)$. By the results of $\S 2$, there is a $\vartheta$-structure

$$
\overline{f_{2}}: \mathscr{G}\left(\bar{L}^{2}\right) \stackrel{\sim}{\longrightarrow} \mathscr{G}^{S}(2 \delta)
$$

such that the pair $\bar{f}_{1}$ and $\bar{f}_{2}$ of $\vartheta$-structures on $X$ is symmetric. Lift $\bar{f}_{2}$ to a $\vartheta$-structure $f_{2}$ for $L^{2}$. Then $f_{1}$ and $f_{2}$ induce isomorphisms:

$$
\begin{gathered}
\Gamma(\mathscr{X}, L) \cong V_{\delta} \otimes_{Z} A \\
\Gamma\left(\mathscr{X}, L^{2}\right) \cong V_{2 \delta} \otimes_{Z} A,
\end{gathered}
$$

where the first is the isomorphism used to define $i: \mathscr{X} \hookrightarrow \boldsymbol{P}\left(V_{\delta}\right) \times S$. Also, evaluation of sections at $\varepsilon$ defines particular $A$-valued functions $q_{L}$ and $q_{L^{2}}$ on $K(\delta)$ and $K(2 \delta)$ via

$$
\begin{array}{cc}
\Gamma\left(\mathscr{X}, L^{i}\right) \cong V_{i \delta} \otimes_{\mathrm{Z}} A \\
\downarrow_{\downarrow^{*}} & \downarrow^{f} f \mapsto \sum_{a \in K(i \delta)} f(a) q_{L^{i}}(a) . \\
A & =A
\end{array}
$$

Here $q_{L}(a)$ is just the value of $Q(a)$ at $\varepsilon$ : so $\left\{q_{L}(a)\right\}$ is a set of homogeneous coordinates for the point $i \circ \varepsilon: S \rightarrow \boldsymbol{P}\left(V_{\delta}\right) \times S$. This means that $\left\{q_{L}(a)\right\}$ are the homogeneous coordinates of $j(S)$, and these are exactly the numbers which are to satisfy RIEMANN's theta relations.

The only thing that must really be checked is that the pairing

$$
\Gamma(\mathscr{X}, L) \times \Gamma(\mathscr{X}, L) \rightarrow \Gamma\left(\mathscr{X}, L^{2}\right)
$$

given by multiplying sections is defined by the same multiplication formula as in $\S 3$. But the proof given in $\S 3$ of this formula goes over without any change to this more general case: simply replace the ground field $k$ by the ground ring $A$. Once the multiplication formula is known, RIEMANN's relations on $\left\{q_{L}(a)\right\}$ follow formally, as in $\S 3$. This proves (i).

To prove (ii), the first point is that $\mathscr{X} \subset \mathscr{Y}$. This follows immediately from the multiplication formula. In fact, on $\mathscr{X}$, the coordinates $Q(a)$ have the constant values $q_{L}(a)$ and the coordinates $X_{a}$ induce the sections of $L$ on $\mathscr{X}$ denoted by $\delta_{a}$ previously. The identity which we need comes out immediately by reducing the quadratic expressions in the $\delta_{a}$ 's to 
functions on $K(2 \delta)$ and using the relation between $q_{L}$ and $q_{L^{2}}$. Secondly, if $Y$ is the fibre of $\mathscr{Y}$ over $k$, then $X=Y$. This follows from Theorem 2, $\S 4$, and the fact that the equations on $X_{a}$ are a complete set of quadratic relations. In fact, for fixed $l \in Z_{2}, a \in K(2 \delta)$, these relations assert that all the quadratic expressions

$$
\left\{\sum_{\eta \in z_{1}} l(\eta) X_{a+b+\eta} \cdot X_{a-b+\eta} \mid b \in a+K(\delta)\right\}
$$

are proportional. Since, in fact, on $X$ each of these sets consists in sections of $L^{2}$ corresponding to the function $Y_{a, l}$ :

$$
Y_{a, l}(b)= \begin{cases}0 & \text { if } b-a \notin Z_{2} \\ l(b-a) & \text { if } b-a \in Z_{2},\end{cases}
$$

and since the $Y_{a . l}$ 's are linearly independent, these do exhaust the quadratic relations on the $X_{a}$. Thirdly, we note the obvious:

Lemma. Let $U$ be a closed subscheme of $V$, both being noetherian schemes over $\operatorname{Spec}(A)$. Then if

1. $U$ is flat over $A$,

2. the fibres $\bar{U}$ and $\bar{V}$ over $\operatorname{Spec}(k)$ are equal, then $U=V$.

This proves (ii).

Step $I V$. Suppose that $N$ is a connected subscheme of $\bar{M}_{\delta}$ such that if $\bar{A}_{\delta}$ induces a subscheme $B$ over $N$, then $B$ is smooth over $N$. Suppose that some geometric point $x$ of $N$ is in the image of $\mathscr{M}_{\delta}$ under $t$. Then the $N$-valued point $1_{N}$ of $N$ is in the image of $\mathscr{M}_{\delta}$, i.e., there is an abelian scheme $\mathscr{Y} \mid N$ with $\delta$-marking such that $i(\mathscr{Y})=B$.

By assumption, one geometric fibre $\bar{B}$ of $B / N$ is an abelian variety. Therefore, by Theorem 6.14, Ch. 6, [9], $B$ is an abelian scheme over $N$ with identity section $\Delta$. By construction, $B \subset \boldsymbol{P}\left(V_{\delta}\right) \times N$. Let $\underline{O}_{\boldsymbol{P}}(1)$ induce the sheaf $M$ on $B$. Normalize it on the identity section, i.e., set

$$
L=M \otimes p_{2}^{*} \Delta^{*}\left(M^{-1}\right)
$$

I claim that the inverse $l$ for $B$ is given by restricting the projective transformation $j$ to $B$, where

$$
\begin{gathered}
j: \boldsymbol{P}\left(V_{\delta}\right) \times N \rightarrow \boldsymbol{P}\left(V_{\delta}\right) \times N \\
j^{*}\left(X_{a}\right)=X_{-a} .
\end{gathered}
$$

But the inverse is given by $j$ on $\bar{B}$ (cf. $\S 3$ ). Since $l=j$ on one geometric fibre of $B \mid N$ and on the section $\Delta$ of $B \mid N, l=j$ everywhere by the rigidity lemma ([9], Ch. 6, §1). Since $j$ is a projective transformation, this shows that $\imath^{*} L \cong L$, i.e., $L$ is symmetric. Now $j$ has 2 disjoint subspaces of fixed 
points:

$$
\begin{array}{llll}
L_{1} & \text { defined by } & X_{a}=X_{-a}, & \text { all } a \in K(\delta) \\
L_{2} & \text { defined by } & X_{a}=-X_{-a}, & \text { all } a \in K(\delta)
\end{array}
$$

and the identity section $\Delta$ is completely contained in $L_{1}$. To say that $L$ is totally symmetric is the same as saying that $B_{2}$, the kernel of multiplication by 2 , is completely contained in $L_{1}$. But $B_{2} \subset L_{1} \cup L_{2}$ since $j=i d$ on $B_{2}$; and $\bar{B}_{2}$, the points of order 2 on $\bar{B}$, is contained in $L_{1}$ since the projective embedding of $\bar{B}$ is assumed to come from a $\delta$-marking. Since $S$ is connected, this implies that $B_{2} \subset L_{1}$, and hence that $L$ is totally symmetric.

Moreover, by Step I, we get a homomorphism

$$
\lambda: \underline{G}(\delta) \times N \rightarrow \mathscr{G}(L)
$$

of group schemes over $N$ such that the actions on $p_{2, *} L$ and $V_{\delta}$ match up. Since $\lambda$ induces an isomorphism of the group schemes $\mathscr{G}(\delta) \times \operatorname{Spec}(k)$, $\mathscr{G}(\bar{L})$ corresponding to the situation in the geometric fibre $\bar{B}, \lambda$ is an isomorphism everywhere ${ }^{2}$. Therefore, $\lambda$ is a $\vartheta$-structure. Also $\lambda$ is symmetric at one point, hence it is symmetric everywhere. Therefore, $B / N$ has a ९-marking which induces the given embedding in $\boldsymbol{P}\left(V_{\delta}\right) \times N$. Q.E.D.

Step $V$. Recall the main result on flattening stratifications ([10], Lecture 8):

Given a projective morphism $f: X \rightarrow Y$ of noetherian schemes, $Y$ can be decomposed into a disjoint union of locally closed connected subschemes $Y_{\alpha}$, such that if $g: Y^{\prime} \rightarrow Y$ is a morphism from a connected noetherian scheme $Y^{\prime}$ to $Y$, and we look at the fibre product:<smiles>[X][Y]1[Y][Y][Y]1[H]</smiles>

then $f^{\prime}$ is flat if and only if $g$ factors through one of the subschemes $Y_{\alpha}$. Apply this to $\bar{A}_{\delta} / \bar{M}_{\delta}$ : if $Z \subset \bar{M}_{\delta}$ is one of the pieces, and some fibre of $\bar{A}_{\delta}$ over $Z$ is obtained from an abelian scheme with $\delta$-marking, then we must show that $Z$ is open. If we do this, both theorems are proven. The openness of $Z$ follows from:

Key Lemma. Let $X / \mathrm{Spec}(k)$ be an abelian variety with $\delta$-marking over an algebraically closed field $k$. Let this define a geometric point $x$ of $\bar{M}_{\delta}$.

\footnotetext{
${ }^{2}$ In fact, since $\lambda$ is an isomorphism of the subgroups $G_{m, S}$ in any case, it is only a question of whether $\lambda$ is an isomorphism of the quotients $\underline{H}(\delta), \underline{H}(L)$. Since these are etale over $S$, a homomorphism is an isomorphism if it is so at one point.
} 
Let $A$ be any artin local ring with residue field $k$, and let

$$
f: \operatorname{Spec}(A) \rightarrow \bar{M}_{\delta}
$$

be a morphism extending $x$. Then the scheme over $\operatorname{Spec}(A)$ induced by $\bar{A}_{\delta}$ is flat over $\operatorname{Spec}(A)$.

Proof of Lemma. Let $\bar{A}_{\delta}$ define, over $A$, the projective scheme

$$
\mathscr{X} \subset \boldsymbol{P}\left(V_{\delta}\right) \times \operatorname{Spec}(A) \text {. }
$$

Let $L$ be the invertible sheaf on $\mathscr{X}$ obtained by restricting $\underline{O}_{P}(1)$ to $\mathscr{X}$. We shall show:

(*) $\quad \quad \quad \quad\left(\mathscr{X}, \mathrm{L}^{2^{2 n}}\right) \quad$ is a free $A$-module, for all large $n$.

This certainly is enough to prove the lemma, in view of the following elementary observation:

Sublemma. Let $A$ be an artin local ring with residue field $k$, and let $R$ be a graded A-algebra generated over $A=R_{0}$ by $R_{1}$. Assume that $R_{1}$ is a finite A-module, and that $R_{n}$ is a free A-module for an infinite set of integers $n$. Then $R_{n}$ is free for all but a finite set of $n$ 's.

Proof. Without loss of generality, we can assume that $k$ is infinite. Let $\bar{R}=R \otimes_{A} k$. In $\bar{R}$, let

$$
\text { (0) }=Q_{1} \cap \cdots \cap Q_{l}, \quad P_{i}=V \bar{Q}_{i}
$$

be the primary decomposition of (0). Let $P_{1}$ be the irrelevant ideal

$$
\underset{n \geqq 1}{\oplus} \bar{R}_{n} \text {. }
$$

Since $k$ is infinite, there is an element $g \in R_{1}$ such that

$$
\overline{\mathrm{g}} \notin P_{2} \cup \cdots \cup P_{l} \text {. }
$$

Let $n_{0}$ be an integer such that $Q_{1} \supset P_{1}^{n_{0}}$, i.e., $Q_{1} \supset R_{n}$ for all $n \geqq n_{0}$. I claim that $R_{m}$ is a free $A$-module for any integer $m \geqq n_{0}$. Choose $f_{1}, \ldots, f_{k}$ in $R_{m}$ such that $\bar{f}_{1}, \ldots, \bar{f}_{k}$ are a basis of $\bar{R}_{m}$. Note that for all integers $p$,

$$
\bar{g}^{p} \bar{f}_{1}, \ldots, \bar{g}^{p} \bar{f}_{k} \in \bar{R}_{m+p}
$$

are independent over $k$. In fact, if

$$
0=\sum \alpha_{i} \bar{g}^{p} \bar{f}_{i}=\bar{g}^{p} \cdot\left(\sum \alpha_{i} \bar{f}_{i}\right)
$$

then $\overline{\mathrm{g}} \notin P_{i}$, for $i \geqq 2$ implies $\sum \alpha_{i} \bar{f}_{i} \in Q_{i}, i \geqq 2$. And since the degree is large enough, $\sum \alpha_{i} \bar{f}_{i} \in Q_{1}$. Hence $\sum a_{i} \bar{f}_{i}=0$, which is a contradiction 
unless all the $\alpha$ 's are 0 . Now suppose that $R_{m}$ is not a free $A$-module. Then

$$
\sum_{i=1}^{k} a_{i} f_{i}=0
$$

for some elements $a_{1}, \ldots, a_{k} \in A$, not all 0 . Let $p$ be a positive integer such that $R_{m+p}$ is a free $A$-module. Then since $\bar{R}_{m+p}$ has a basis of the form $\bar{g}^{p} \bar{f}_{1}, \ldots, \bar{g}^{p} \bar{f}_{k}, \bar{h}_{1}, \ldots, \bar{h}_{l}$, it follows that $R_{m+p}$ has a basis of the form $g^{p} f_{1}, \ldots, g^{p} f_{k}, h_{1}, \ldots, h_{l}$. But

$$
\sum_{i=1}^{k} a_{i}\left(f_{i} g^{p}\right)=0
$$

Therefore, the $a$ 's are all 0 which is a contradiction. Q.E.D.

Once we know that $\Gamma\left(\mathscr{X}, L^{n}\right)$ is a free $A$-module for all large $n$, then $\mathscr{X}$ is flat over $\operatorname{Spec}(A)\left([5]\right.$, Ch. 3, 7.9.14). To prove $(*)$, let $I_{\delta} \subset S^{*}\left(V_{\delta}\right) \otimes A$ be the ideal generated by the quadratic defining relations of $\bar{A}_{\delta}$. Then

$$
\Gamma\left(\mathscr{X}, L^{n}\right) \cong\left(S^{n} V_{\delta} \otimes A\right) /\left(I_{\delta}\right)_{n}
$$

for all large $n$, and it suffices to prove:

$$
\begin{array}{ll}
\left(I_{\delta}\right)_{2^{n}} & \text { is a direct summand of } \quad\left(S^{2^{n}} V_{\delta}\right) \otimes A \\
& \text { for all } n=0,1,2, \ldots .
\end{array}
$$

We shall prove (**) by induction on $n$, assuming at each stage that it is known for $n \leqq n_{0}$ and all $\delta$. To start things off, $\left(I_{\delta}\right)_{1}$ equals $(0)$, for $(* *)$ is always true for $n=0$. To get from (**) for $n=n_{0}$ to $(* *)$ for $n=n_{0}+1$, we shall simply show for all $A$-valued points of $\bar{M}_{\delta}$ as above, there is an $A$-valued point of $\bar{M}_{2 \delta}$ with the same properties such that:

$(* * *)$

In particular,

$$
\underset{n=0}{\infty}\left(\left(S^{2 n} V_{\delta}\right) \otimes A\right) /\left(I_{\delta}\right)_{2 n} \cong\left(\left(S^{*} V_{2 \delta}\right) \otimes A\right) / I_{2 \delta} .
$$

$$
\left(\left(S^{2 n+1} V_{\delta}\right) \otimes A\right) /\left(I_{\delta}\right)_{2^{n+1}} \cong\left(S^{2^{n}} V_{2 \delta} \otimes A\right) /\left(I_{2 \delta}\right)_{2^{n}}
$$

so $(* *)$ will be completely proven.

To lift the $A$-valued point of $\bar{M}_{\delta}$, we use the morphism

$$
\pi: \bar{M}_{2 \delta} \rightarrow \bar{M}_{\delta}
$$

defined in Step II. The underlying $k$-valued point of $f$ corresponds to an abelian variety with $\delta$-marking. Choose a compatible $2 \delta$-marking on this abelian variety. This defines a $k$-valued point $y$ of $\bar{M}_{2 \delta}$ over $x$. By Step II, $\pi$ is étale at $x$. Therefore, there is one (and only one) $A$-valued point

$$
g: \operatorname{Spec}(A) \rightarrow \bar{M}_{2 \delta}
$$

lifting $f$ with underlying $k$-valued point $y$. 
Let these $A$-valued points be given by homogeneous coordinates $q(a), a \in K(\delta)$, and $q^{\prime}(b), b \in K(2 \delta)$, where $q(a), q^{\prime}(b) \in A$. These satisfy the usual equations (cf. Step II). Then in concrete terms:

$$
\begin{aligned}
& S^{*} V_{\delta} \otimes A / I_{\delta} \\
& \cong \frac{A\left[\ldots, X_{a}, \ldots\right]_{a \in K(\delta)}}{\left\{\begin{array}{r}
\left(\sum l(\eta) q(c+d+\eta) \cdot q(c-d+\eta)\right) \cdot\left(\Sigma l(\eta) X_{a+b+\eta} X_{a-b+\eta}\right) \\
-\left(\sum l(\eta) q(c+b+\eta) \cdot q(c-b+\eta)\right) \cdot\left(\sum l(\eta) X_{a+d+\eta} X_{a-d+\eta}\right)
\end{array}\right\}} \\
& S^{*} V_{2 \delta} \otimes A / I_{2 \delta} \\
& \cong \frac{A\left[\ldots, X_{a}^{\prime}, \ldots\right]_{a \in K(2 \delta)}}{\left\{\begin{array}{r}
\left(\sum l(\eta) q^{\prime}(c+d+\eta) \cdot q^{\prime}(c-d+\eta)\right) \cdot\left(\Sigma l(\eta) X_{a+b+\eta}^{\prime} X_{a-b+\eta}^{\prime}\right) \\
-\left(\sum l(\eta) q^{\prime}(c+b+\eta) \cdot q^{\prime}(c-b+\eta)\right) \cdot\left(\sum l(\eta) X_{a+d+\eta}^{\prime} X_{a-d+\eta}^{\prime}\right)
\end{array}\right\}} .
\end{aligned}
$$

We set up the isomorphism $(* * *)$ by requiring that

$$
T\left[X_{a+b} \cdot X_{a-b}\right]=\sum_{\eta \in Z_{2}} q^{\prime}(b+\eta) \cdot X_{a+\eta}^{\prime}
$$

for all $a, b \in K(2 \delta)$ such that $a+b \in K(\delta)$. This implies:

$$
T\left[\sum_{\eta \in Z_{2}} l(\eta) X_{a+b+\eta} X_{a-b+\eta}\right]=\sum_{\eta \in Z_{2}} l(\eta) q^{\prime}(b+\eta) \cdot \sum_{\eta \in Z_{2}} l(\eta) X_{a+\eta}^{\prime}
$$

for all $a, b \in K(2 \delta), l \in \hat{Z}_{2}$ such that $a+b \in K(\delta)$. Since

$$
\Sigma l(\eta) q(a+b+\eta) q(a-b+\eta)=\Sigma l(\eta) q^{\prime}(a+\eta) \cdot \Sigma l(\eta) q^{\prime}(b+\eta),
$$

this second equation implies that the quadratic relations in the ring of $X$ 's go to 0 in the ring of $X^{\prime}$ 's. Moreover, since for all $a \in K(2 \delta), l \in \hat{Z}_{2}$, there is an element $b \in a+K(\delta)$ such that $\sum\left(l(\eta) q^{\prime}(b+\eta)\right)$ is a unit, this also shows that the map is surjective. Similarly, since the elements $\sum l(\eta) X_{a+\eta}^{\prime}$ are linearly independent it is clear from this second equation that every quadratic expression in the $X$ 's which goes to 0 via $T$ is a combination of expressions of the form:

$$
\sum_{b \in a+K(\delta)} A_{b}\left\{\sum_{\eta \in Z_{2}} l(\eta) X_{a+b+\eta} X_{a-b+\eta}\right\} .
$$

And, in fact, the expressions

$$
\begin{aligned}
& {\left[\Sigma l(\eta) q^{\prime}\left(b_{2}+\eta\right)\right] \cdot\left[\Sigma l(\eta) X_{a+b_{1}+\eta} \cdot X_{a-b_{1}+\eta}\right]} \\
& \quad-\left[\Sigma l(\eta) q^{\prime}\left(b_{1}+\eta\right)\right] \cdot\left[\Sigma l(\eta) X_{a+b_{2}+\eta} X_{a-b_{2}+\eta}\right]
\end{aligned}
$$

will span the kernel. But if $b_{1}=b, b_{2}=d$ and we multiply this expression by a unit of the form $\sum l(\eta) q^{\prime}(c+\eta), c \in a+K(\delta)$, we get the typical quadratic relation on the $X$ 's. Therefore, $T$ sets up an isomorphism:

$$
S^{2} V_{\delta} \otimes A /\left(I_{\delta}\right)_{2} \stackrel{\sim}{\longrightarrow} S^{1} V_{2 \delta} \otimes A /\left(I_{2 \delta}\right)_{1} \text {. }
$$


At this stage in the proof, we have already shown that for all $\delta,\left(I_{\delta}\right)_{2}$ is a direct summand of $S^{2} V_{\delta} \otimes A$.

Now try to extend $T$ to a homomorphism of the whole ring. Let $U$ be the free $A$-module $S^{2} V_{\delta} \otimes A /\left(I_{\delta}\right)_{2}$. Let $Y_{a, b}$ be the element $X_{a} X_{b}$ in $U$. Then, as observed in the proof of Theorem $2, \S \mathrm{IV}$,

$$
\bigoplus_{n=0}^{\infty} S^{2 n} V_{\delta} \otimes A /\left(I_{\delta}\right)_{2 n} \cong S^{*} U /\left\{\begin{array}{c}
\text { ideal generated by } \\
Y_{a, b} Y_{c, d}-Y_{a, d} Y_{c, b}
\end{array}\right\} .
$$

Therefore, all we have to check is that when $T$ is extended to $S^{2} U$, the $A$-module generated by the expressions $T\left(Y_{a, b}\right) \cdot T\left(Y_{c, d}\right)-T\left(Y_{a, d}\right) \times$ $T\left(Y_{c, b}\right)$ is the same as the $A$-module of quadratic relations in the $X^{\prime}$ 's, and the proof will be complete. Call the first module $N_{1}$, and the second $N_{2}$. It is easy to check that $N_{1} \subset N_{2}$, simply by calculating out the expressions $T\left(Y_{a, b}\right) \cdot T\left(Y_{c, d}\right)-T\left(Y_{a, d}\right) \cdot T\left(Y_{c, b}\right)$.

Consider the diagram

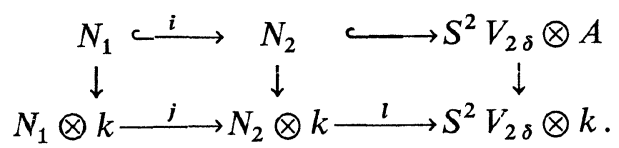

Since $N_{2}$ is a direct summand of $S^{2} V_{2 \delta} \otimes A$ according to the first part of this proof, it follows that $l$ is injective. But in Theorem $2, \S 4$, we showed that $N_{1}=N_{2}$ in case $A=k$. Therefore the images of $N_{1} \otimes k$ and $N_{2} \otimes k$ in $S^{2} V_{2 \delta} \otimes k$ are equal. This implies that $j$ is surjective, hence that $i$ is surjective, and hence $N_{1}=N_{2}$. Q.E.D.

\section{§7. The 2-Adic Limit}

Up to this point, we have been studying pairs $(X, L)$, consisting of abelian varieties $X$, and ample invertible sheaves $L$ of separable type. To push the theory further, however, it seems almost essential to make it freer of variations within one isogeny type. The simplest way to do this is to study simultaneously a whole tower of abelian varieties. All the simplifications that occur however have to do with dividing by 2 , so it doesn't seem necessary or fruitful to look at all isogenies: instead we look only at isogenies of degree $2^{n}$, some $n$. This is economical, too, because our moduli will then be the values of functions on 2-adic vector spaces, rather than of functions on adelic spaces. As always, we assume $\operatorname{char}(k) \neq 2$.

The basic definition is:

Definition 1. A 2-tower of abelian varieties (or tower) is an inverse system $\left\{X_{\alpha}\right\}_{\alpha \in S}$ of abelian varieties, i.e., $S$ is a partially ordered set such that $\forall \alpha_{1}, \alpha_{2} \in S, \exists \beta \in S, \beta>\alpha_{1}, \alpha_{2}$, and whenever $\alpha, \beta \in S, \alpha>\beta$, we are 
given an isogeny of degree $2^{n}$ :

such that:

$$
p_{\alpha, \beta}: X_{\alpha} \rightarrow X_{\beta}
$$

1) If $\alpha>\beta>\gamma$, the diagram of isogenies

commutes,

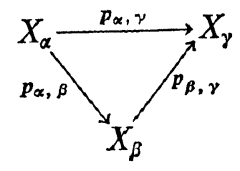

2) If $\alpha>\beta_{1}, \beta_{2}$ and $K_{i}=\operatorname{kernel}\left\{X_{\alpha} \rightarrow X_{\beta_{i}}\right\}$, then $K_{1} \subseteq K_{2} \Leftrightarrow \beta_{1} \geqq \beta_{2}$.

3a) For all $\alpha \in S$, and all isogenies $X_{\alpha} \rightarrow Y$, of degree $2^{n}$ (some $n$ ), $\exists \beta \in S$ such that $\alpha \geqq \beta$, and one has a diagram:<smiles>[X]C1[Y4]C#[Y]1</smiles>

3b) For all $\alpha \in S$, and all isogenies $Y \rightarrow X_{\alpha}$ of degree $2^{n}$ (some $n$ ), $\exists \beta \in S$ such that $\beta \geqq \alpha$ and one has a diagram:<smiles></smiles>

Note that, starting with one abelian variety $X$, we can generate in a canonical way a 2-tower of abelian varieties by taking coverings and quotients of degrees $2^{n}$, starting with $X$.

Definition 2. If $X$ is an abelian variety, let $\operatorname{tor}_{2}(X)$ denote the group of closed points $x \in X$ of order $2^{n}$, some $n$.

If $X=\left\{X_{\alpha}\right\}$ is a tower of abelian varieties, we get a derived inverse system of discrete groups $\left\{\operatorname{tor}_{2}\left(X_{\alpha}\right)\right\}$. Let

$$
V\left(\underset{\leftarrow}{X)}=\varliminf_{\alpha \in S} \operatorname{tor}_{2}\left(X_{\alpha}\right)\right. \text {. }
$$

If $X$ is one abelian variety, we also let $V(X)$ denote $V(X)$, where $X$ is the 2-tower generated by $X$. This is the usual 2-Tate group of $X$. For all $\alpha \in S$, there is a canonical surjection

$$
p_{\alpha}: V(\underset{+}{X}) \rightarrow \operatorname{tor}_{2}\left(X_{\alpha}\right)
$$


Denote the kernel by $T(\alpha)$. Each $T(\alpha)$ is an inverse limit of finite groups, so if we topologize $V(\underline{X})$ by taking the $T(\alpha)$ 's as a basis of open neighborhoods of the origin, then $V(\underline{X})$ becomes a locally compact group, and each $T(\alpha)$ becomes a compact, open subgroup. From well-known structure theorems, we know that there are topological isomorphisms:

(where $g=\operatorname{dim} X$ ). In particular,

$$
\begin{aligned}
& 0 \longrightarrow T(\alpha) \longrightarrow V(\underset{\leftarrow}{X}) \longrightarrow \text { tor }_{2}\left(X_{\alpha}\right) \longrightarrow 0
\end{aligned}
$$

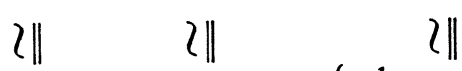

$$
\begin{aligned}
& 0 \longrightarrow\left(Z_{2}\right)^{2 g} \longrightarrow\left(Q_{2}\right)^{2 g} \longrightarrow\left\{\begin{array}{l}
\text { subgroup of } \\
Q / Z \text { of elts. } \\
\text { of order } 2^{n} \\
\text { (some } n \text { ) }
\end{array}\right\}^{2 g} \longrightarrow 0
\end{aligned}
$$

$$
S \cong\left\{\begin{array}{l}
\text { partially ordered set of compact, } \\
\text { open subgroups } T \subset\left(Q_{2}\right)^{2 g}
\end{array}\right\} .
$$

When $X$ is generated from one given $X$, the kernel of the canonical homomorphism $V(X) \rightarrow$ tor $_{2}(X)$ is usually denoted $T(X)$, and $T(X)$ is also called the 2-Tate group of $X$.

A final point: when $k=C$, one can associate to a tower of abelian varieties a common universal covering space. In fact, for all $\alpha \geqq \beta, p_{\alpha \beta}$ : $X_{\alpha} \rightarrow X_{\beta}$ induces an isomorphism

$$
\hat{p}_{\alpha \beta}: \hat{X}_{\alpha} \stackrel{\sim}{\longrightarrow} \hat{X}_{\beta}
$$

between the universal covering spaces. Therefore let $\hat{X}$ stand for a complex vector space canonically isomorphic to each $\hat{\hat{X}}_{\alpha}$. In the classical theory, $\hat{X}$ plays a role analogous to $V(X)$. For all $\alpha$, there is a canonical surjection $q_{\alpha}: \hat{X} \rightarrow X_{\alpha}$. Let $L(\alpha)$ denote its kernel. Then $\{L(\alpha)\}$ is a family of commensurable lattices in $\underline{X}$, and $\alpha \geqq \beta$ if and only if $L(\alpha) \subseteq L(\beta)$. Since $X_{\alpha} \cong \hat{X} / L(\alpha)$, the whole tower can be generated by starting with the complex vector space $\hat{X}$ and dividing by these lattices $L(\alpha)$.

Definition 3. A polarized tower of abelian varieties is a tower $\left\{X_{\alpha}\right\}$, $\alpha \in S$, plus a set of totally symmetric ample invertible sheaves of degree $2^{n}$, some $n: L_{\alpha}$ on $X_{\alpha}$, for $\alpha$ in a subset $S_{0} \subset S$, plus isomorphisms

$$
p_{\alpha, \beta}^{*}\left(L_{\beta}\right) \stackrel{\sim}{\longrightarrow} L_{\alpha}
$$

whenever $\alpha, \beta \in S_{0}, \alpha>\beta$. We require:

1) If $\alpha, \beta \in S$, and $\alpha>\beta$, then $\beta \in S_{0} \Rightarrow \alpha \in S_{0}$.

2) If $\alpha>\beta>\gamma, \gamma \in S_{0}$, then the isomorphisms of $L_{\alpha}, L_{\beta}, L_{\gamma}-$ all pulled up to $X_{\alpha}-$ are to be compatible, 
3) If $\alpha>\beta, \alpha \in S_{0}$, and if there exists a totally symmetric sheaf $M$ on $X_{\beta}$ such that $p_{\alpha, \beta}^{*} M \cong L_{\alpha}$, then $\beta \in S_{0}$ too, [in which case, $L_{\beta}$ will have to be $M$ too].

The object of this section is to generalize the theory of $\S 1$ to a polarized tower of abelian varieties. So from now on, let's suppose given one such tower $\mathscr{T}=\left\{X_{\alpha}, L_{\alpha}\right\}$. The first important observation is:

"4>2" Lemma. For all $x \in V(X)$, there exists an $\alpha \in S_{0}$ such that $p_{\alpha}(x) \in H\left(L_{\alpha}\right)$.

Proof. Start with any $\alpha_{1} \in S_{0}$. Let $2^{n}$ be the order of the point $p_{\alpha_{1}}(x)$. Let $\alpha_{2} \in S_{0}$ be the element such that $\alpha_{2}>\alpha_{1}$ and such that the isogeny $p_{\alpha_{2}, \alpha_{1}}$ is $2^{n} \delta$, i.e.,

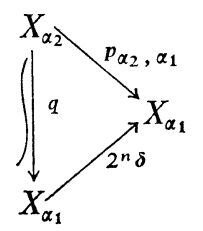

Since $\left(2^{n} \delta\right)^{*} L_{\alpha_{1}} \cong\left(L_{\alpha_{1}}\right)^{22 n}, H\left(\left(2^{n} \delta\right)^{*} L_{\alpha_{1}}\right)$ contains all points of $X_{\alpha_{1}}$ of order $2^{2 n}$. Therefore $H\left(L_{\alpha_{2}}\right)$ contains all points of $X_{\alpha_{2}}$ of order $2^{2 n}$. But

$$
0=2^{n} p_{\alpha_{1}}(x)=2^{n} p_{\alpha_{2}, \alpha_{1}}\left(p_{\alpha_{2}}(x)\right)=2^{2 n}\left[q\left(p_{\alpha_{2}}(x)\right)\right]
$$

so $p_{\alpha_{2}}(x)$ has order $2^{2 n}$. Q.E.D.

Now let $S_{0}^{x}=\left\{\alpha \in S_{0} \mid p_{\alpha}(x) \in H\left(L_{\alpha}\right)\right\}$. Like $S_{0}$, it has the property: $\beta>\alpha, \alpha \in S_{0}^{x} \Rightarrow \beta \in S_{0}^{x}$. Suppose that for one $\alpha \in S_{0}^{x}$, you choose an isomorphism

$$
\varphi_{\alpha}: L_{\alpha} \stackrel{\sim}{\longrightarrow} T_{p_{\alpha}(x)}^{*}\left(L_{\alpha}\right)
$$

Then $I$ claim this determines canonically isomorphisms

$$
\varphi_{\beta}: L_{\beta} \stackrel{\sim}{\longrightarrow} T_{p_{\beta}(x)}^{*}\left(L_{\beta}\right)
$$

for all $\beta \in S_{0}^{x}$. This is clear - first suppose $\beta>\alpha$, and then choose $\varphi_{\beta}$ so that:

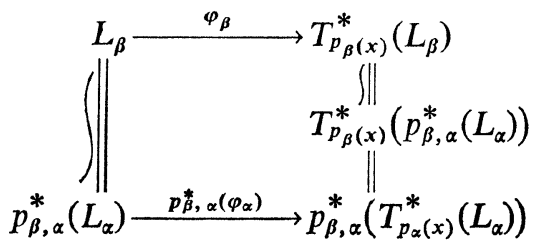

commutes. In general, first determine $\varphi_{\gamma}$ for some $\gamma \in S_{0}^{x}$ such that $\gamma>\alpha, \beta$, then go backwards to determine $\varphi_{\beta}$ from $\varphi_{\gamma}$. Call such a system of $\varphi_{\alpha}$ 's, all $\alpha \in S_{0}^{x}$, a compatible set of isomorphisms $L_{\alpha} \underset{\longrightarrow}{\longrightarrow} T_{p_{\alpha}(x)}^{*}\left(L_{\alpha}\right)$. 
Definition 4. $\mathscr{G}(\mathscr{T})=$ set of pairs $\left(x,\left\{\varphi_{\alpha}\right\}\right)$, where $x \in V(\underline{X})$, and $\left\{\varphi_{\alpha}\right\}, \alpha \in S_{0}^{x}$, is a compatible set of isomorphisms.

This forms a group in the usual way. Given $\left(x,\left\{\varphi_{\alpha}\right\}\right),\left(y,\left\{\psi_{\alpha}\right\}\right) \in \mathscr{G}(\mathscr{T})$, choose any $\gamma \in S_{0}^{x} \cap S_{0}^{y}$. Then form the composition:

$$
L_{\gamma} \underset{\psi_{\gamma}}{\longrightarrow} T_{p_{\gamma}(y)}^{*}\left(L_{\gamma}\right) \underset{T_{p_{\gamma}(y)}^{*}\left(\varphi_{\gamma}\right)}{\longrightarrow} T_{p_{\gamma}(x+y)}^{*}\left(L_{\gamma}\right) .
$$

Call this $\rho_{\gamma}$ and generate with it a compatible set of isomorphisms $\left\{\rho_{\alpha}\right\}$, all $\alpha \in S_{0}^{x+y}$. Then let

$$
\left(x,\left\{\varphi_{\alpha}\right\}\right) \circ\left(y,\left\{\psi_{\alpha}\right\}\right)=\left(x+y,\left\{\rho_{\alpha}\right\}\right) .
$$

Moreover, we get an exact sequence:

$$
0 \longrightarrow k^{*} \longrightarrow \mathscr{G}(\mathscr{T}) \stackrel{\pi}{\longrightarrow} V(\underset{\leftarrow}{X}) \longrightarrow 0
$$

as usual. Notice the simplification here over the theory of $\S 1$ : the finite group $H(L)$ that depended on $L$ has been replaced by the big group $V(X)$ depending only on the tower and not on the polarization. It is not hard to interpret $\mathscr{G}(\mathscr{T})$ as a simultaneous direct and inverse limit of the $\mathscr{G}\left(L_{\alpha}\right)$ 's, with respect to the connections induced between them whenever $\alpha>\beta$, as in Prop. $2, \S 1$. In particular, for all $\alpha_{1} \in S_{0}$, if we look at the subgroup:

$$
\bigcap_{\mathscr{G}(\mathscr{T})}^{\mathscr{G}_{\alpha_{1}}^{*}(\mathscr{T})=\pi^{-1}\left[p_{\alpha_{1}}^{-1}\left(H\left(L_{\alpha_{1}}\right)\right)\right]}
$$

then for all $\left(x,\left\{\varphi_{\alpha}\right\}\right) \in \mathscr{G}_{\alpha_{1}}^{*}(\mathscr{T}), p_{\alpha_{1}}(x) \in H\left(L_{\alpha_{1}}\right)$ so $\varphi_{\alpha_{1}}$ is defined, and

defines a surjection:

$$
\left(x,\left\{\varphi_{\alpha}\right\}\right) \mapsto\left(p_{\alpha_{1}}(x), \varphi_{\alpha_{1}}\right)
$$

$$
\mathscr{G}_{\alpha_{1}}^{*}(\mathscr{T}) \stackrel{q \alpha_{1}}{\longrightarrow} \mathscr{G}\left(L_{\alpha_{1}}\right) \longrightarrow 0 \text {. }
$$

Let $K\left(\alpha_{1}\right)$ denote the kernel of this map. We get the picture:

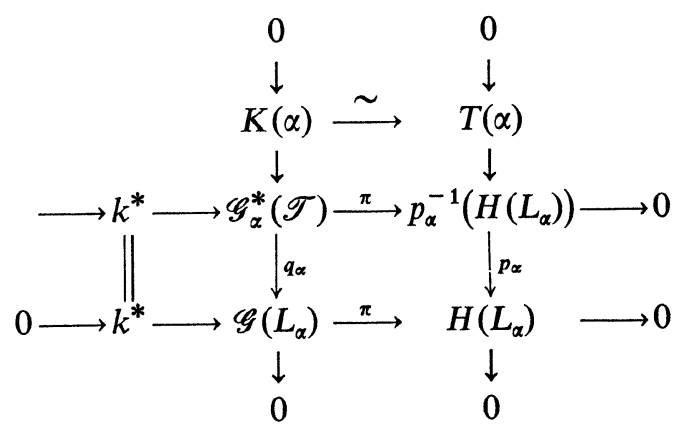


Lemma 2. For all $\alpha \in S_{0}, \mathscr{G}_{\alpha}^{*}(\mathscr{T})$ is the centralizer of $K(\alpha)$ in $\mathscr{G}(\mathscr{T})$, and $k^{*} \cdot K(\alpha)$ is the center of $\mathscr{G}_{\alpha}^{*}(\mathscr{T})$.

Proof. If $x \in K(\alpha)$ and $y \in \mathscr{G}_{\alpha}^{*}(\mathscr{T})$, then $y \cdot x \cdot y^{-1}$ is still in $K(\alpha)$, and it has the same image in $T(\alpha)$ as $x$ has, since $p_{\alpha}^{-1}\left(H\left(L_{\alpha}\right)\right)$ is a commutative group. Therefore $x=y \cdot x \cdot y^{-1}$, i.e., $K(\alpha) \subset$ center $\left[\mathscr{G}_{\alpha}^{*}(\mathscr{T})\right]$. Since $k^{*}$ is even in the center of $\mathscr{G}(\mathscr{T}), k^{*} \cdot K(\alpha) \subset$ center $\left[\mathscr{G}_{\alpha}^{*}(\mathscr{T})\right]$ too. But if $x \in$ center $\left[\mathscr{G}_{\alpha}^{*}(\mathscr{T})\right], q_{\alpha}(x) \in$ center $\left[\mathscr{G}\left(L_{\alpha}\right)\right]$ and we know $k^{*}$ is the whole center of $\mathscr{G}\left(L_{\alpha}\right)$. Therefore $k^{*} \cdot K(\alpha)$ is exactly the center of $\mathscr{G}_{\alpha}^{*}(\mathscr{T})$. Now suppose $y \in \mathscr{G}(\mathscr{T})$ centralized $K(\alpha), y$ is certainly in $\mathscr{G}_{\beta}^{*}(\mathscr{T})$ for some $\beta \in S,(\beta>\alpha)$. Then the image $q_{\beta}(y)$ in $\mathscr{G}\left(L_{\beta}\right)$ commutes with the image $q_{\beta}[K(\alpha)]$ in $\mathscr{G}\left(L_{\beta}\right)$. Now $q_{\beta}[K(\alpha)]$ is a subgroup of $\mathscr{G}\left(L_{\beta}\right)$ lying over the subgroup $\operatorname{Ker}\left(p_{\beta \alpha}\right)$ of $H\left(L_{\beta}\right)$. Therefore by Prop. 2, $\S 1, q_{\beta}(y)$ is an element of $\mathscr{G}\left(L_{\beta}\right)$ whose image in $H\left(L_{\beta}\right)$ is in $p_{\beta, \alpha}^{-1}\left[H\left(L_{\alpha}\right)\right]$, i.e., the image of $y$ in $V(X)$ is in $p_{\alpha, \beta}^{-1}\left(H\left(L_{\alpha}\right)\right)$, or $y \in \mathscr{G}_{\alpha}^{*}(\mathscr{T})$. Q.E.D.

Corollary. $k^{*}=$ center of $\mathscr{G}(\mathscr{T})$.

As in $\S 1$, we can describe the non-commutativity by a skew-symmetric form $e_{\lambda}: V(\underline{X}) \times V(\underline{X}) \rightarrow k^{*}$ :

$$
e_{\lambda}(\pi x, \pi y)=x \cdot y \cdot x^{-1} \cdot y^{-1}
$$

all $x, y \in \mathscr{G}(\mathscr{T})$. Then the lemma tells us that:

1) $p_{\alpha}^{-1}\left(H\left(L_{\alpha}\right)\right)$ is the group of elements $x \in V(\underline{X})$ such that $e_{\lambda}(x, y)=1$, all $y \in T(\alpha)$, i.e., $T(\alpha)^{\perp}$.

2) $T(\alpha)$ is the group of elements $x \in V(X)$ such that $e_{\lambda}(x, y)=1$, all $y \in p_{\alpha}^{-1}\left(H\left(L_{\alpha}\right)\right)$, i.e., the degenerate subspace for the pairing $e_{\lambda}$ on $p_{\alpha}^{-1}\left(H\left(L_{\alpha}\right)\right)$.

3) For all $x \in V(X)$, there is a $y \in V(\underline{X})$ such that $e_{\lambda}(x, y) \neq 1$, i.e., $e_{\lambda}$ is non-degenerate.

In particular, for all $\alpha \in S_{0}$, (a) $e_{\lambda} \equiv 1$ on $T(\alpha)$, (b) $T(\alpha)^{\perp} / T(\alpha) \cong H\left(L_{\alpha}\right)$, and (c) the pairing induced on $T(\alpha)^{\perp} / T(\alpha)$ by $e_{\lambda}$ corresponds to the old pairing $e_{L_{\alpha}}$ on $H\left(L_{\alpha}\right)$.

Using the symmetry of the $L_{\alpha}$ 's, we obtain an automorphism

$$
\delta_{-1}: \mathscr{G}(\mathscr{T}) \rightarrow \mathscr{G}(\mathscr{T})
$$

exactly as in $\S 2$. But now since $2 \cdot V(\underline{X})=V(X), \delta_{-1}$ is much more convenient than before. In fact, it induces a canonical section of $\mathscr{G}(\mathscr{T})$ over $V(X)$ :

Definition 5. Let $x \in V(X)$. Let $z \in \mathscr{G}(\mathscr{T})$ satisfy $\pi(z)=x / 2, \delta_{-1}(z)=z^{-1}$ : there are exactly 2 such elements in $\mathscr{G}(\mathscr{T}), z$ and $(-1) \cdot z$ (here -1 is an 
element of $k^{*}$ and we multiply in $\mathscr{G}(\mathscr{T})$ ). Let

$$
\sigma(x)=z^{2}
$$

(which is independent of the choice of $z$ ).

Therefore, $\mathscr{G}(\mathscr{T})$ decomposes as a set:

if

$$
\begin{gathered}
\mathscr{G}(\mathscr{T}) \cong k^{*} \times V(\underset{\leftarrow}{X}) \\
\alpha \cdot \sigma(x) \leftrightarrow(\alpha, x) .
\end{gathered}
$$

Let's compute what happens to the group law:

Lemma 3. For all $x, y \in V(\underset{\leftarrow}{X}), \sigma(x) \cdot \sigma(y)=e_{\lambda}(x, y / 2) \cdot \sigma(x+y)$.

Proof. Let $z, w \in \mathscr{G}(\mathscr{T})$ lie over $x / 2, y / 2$ respectively and satisfy $\delta_{-1} z=z^{-1}, \delta_{-1} w=w^{-1}$. Let

$$
s=e_{\lambda}(-x / 4, y / 2) \cdot z \cdot w .
$$

Then $s$ lies over $(x+y) / 2$ and satisfies:

$$
\begin{aligned}
\delta_{-1} s & =e_{\lambda}(-x / 4, y / 2) \cdot \delta_{-1} z \cdot \delta_{-1} w \\
& =e_{\lambda}(-x / 4, y / 2) \cdot\left[z^{-1} \cdot w^{-1} \cdot z \cdot w\right] \cdot(z \cdot w)^{-1} \\
& =e_{\lambda}(-x / 4, y / 2) \cdot e_{\lambda}(-x / 2,-y / 2) \cdot(z \cdot w)^{-1} \\
& =s^{-1} .
\end{aligned}
$$

Therefore

$$
\begin{aligned}
e_{\lambda}(x, y / 2) \sigma(x+y) & =e_{\lambda}(x, y / 2) \cdot s^{2} \\
& =e_{\lambda}(x / 2, y / 2) \cdot z \cdot w \cdot z \cdot w \\
& =e_{\lambda}(x / 2, y / 2) \cdot z^{2}\left(z^{-1} \cdot w \cdot z \cdot w^{-1}\right) w^{2} \\
& =\sigma(x) \cdot \sigma(y) .
\end{aligned}
$$

Q.E.D.

In other words, the group law, carried over to $k^{*} \times V(\underset{\leftarrow}{X})$, is

$$
(\alpha, x) \cdot(\beta, y)=\left(\alpha \cdot \beta \cdot e_{\lambda}(x, y / 2), x+y\right) \text {. }
$$

Let's give a complete structure theorem for $\mathscr{G}(\mathscr{T})$ and $V(\underset{\sim}{X})$. Let

$\chi: \boldsymbol{Q}_{2} \rightarrow k^{*} \quad$ be an additive character with kernel $Z_{2}$.

Definition 6. A symplectic isomorphism of $V\left(\underset{X)}{X}\right.$ and $Q_{2}^{g} \times Q_{2}^{g}$ is an isomorphism $\bar{c}$ such that if $\varphi_{1} x, \varphi_{2} x$ are the $1^{\text {st }} g$ and $2^{\text {nd }} g$ components of $\bar{c}(x)$, then

$$
e_{\lambda}(x, y)=\chi\left[{ }^{t} \varphi_{1} x \cdot \varphi_{2} y-{ }^{t} \varphi_{1} y \cdot \varphi_{2} x\right]
$$


for all $x, y \in V(\underline{X})$ (here $t$ denotes the transpose vector, and - is multiplication of $1 \times g$ and $g \times 1$ matrices).

We leave it to the reader to check that such an isomorphism always exists: for example by constructing it inductively via some cofinal series $\alpha_{1}<\alpha_{2}<\cdots$ in $S$.

Definition 7. $\mathscr{G}_{\mathrm{g}}=k^{*} \times \boldsymbol{Q}_{2}^{g} \times \boldsymbol{Q}_{2}^{\mathrm{g}}$, with group law

$$
(\alpha, x, y) \cdot(\beta, u, v)=\left(\alpha \cdot \beta \cdot \chi\left({ }^{t} x \cdot v\right), x+u, y+v\right) .
$$

$D_{-1}: \mathscr{G}_{g} \rightarrow \mathscr{G}_{g}$ is the automorphism $D_{-1}((\alpha, x, y))=(\alpha,-x,-y)$. $\Sigma: \boldsymbol{Q}_{2}^{\mathrm{g}} \times \boldsymbol{Q}_{2}^{\mathrm{g}} \rightarrow \mathscr{G}_{\mathrm{g}}$ is the section

$$
\Sigma((x, y))=\left(\chi\left(\frac{{ }^{t} x \cdot y}{2}\right), x, y\right) .
$$

Definition 8. A full $\vartheta$-structure for $\mathscr{T}$ is an isomorphism $c: \mathscr{G}(\mathscr{T}) \sim \mathscr{G}_{\mathrm{g}}$, which is the identity on the subgroups $k^{*}$ and such that $c \circ \delta_{-1}=D_{-1} \circ c$.

It follows immediately that a full $\vartheta$-structure $c$ induces a symplectic isomorphism $\bar{c}: V(X \underline{X}) \sim \boldsymbol{Q}_{2}^{2}$, and also that $\Sigma \circ \bar{c}=c \circ \sigma$. Therefore $c$ is even determined by $\bar{c}$. This is a simplification which was foreshadowed in the discussion at the end of $\S 2$. In fact, given a symplectic isomorphism $\bar{c}$, we can define the unique full $\vartheta$-structure $c$ extending $\bar{c}$ by:

$$
\begin{aligned}
c(\lambda \cdot \sigma(x)) & =\lambda \cdot \Sigma(\bar{c}(x)) \\
& =\left(\lambda \cdot \chi\left(\frac{{ }^{t} \varphi_{1} x \cdot \varphi_{2}}{2} x\right), \varphi_{1} x, \varphi_{2} x\right)
\end{aligned}
$$

for all $\lambda \in k^{*}, x \in V(\underline{X})$, and one checks all the requirements easily. In particular, one full $\vartheta$-structure always exists and we have a structure theorem for $\mathscr{G}(\mathscr{T})$ and its maps.

So far, in our polarized tower, we have considered only the totally symmetric $L_{\alpha}$ 's that can be put in an inverse system on the $X_{\alpha}$ 's. For a few more $\alpha$ 's, however, we may be able to find a symmetric invertible sheaf $L_{\alpha}$ on $X_{\alpha}$, such that for some element $\beta \in S_{0}$ for which $\beta \geqq \alpha, p_{\alpha, \beta}^{*} L_{\alpha}$ is isomorphic to the $L_{\beta}$ that we already have: such an $L_{\alpha}$ will be said to be compatible with the given polarization.

If we define as before

$$
\mathscr{G}_{\alpha}^{*}(\mathscr{T})=\pi^{-1}\left(p_{\alpha}^{-1}\left(H\left(L_{\alpha}\right)\right)\right),
$$

then every element $\left(\left\{x_{\beta}\right\},\left\{\varphi_{\beta}\right\}\right) \in \mathscr{G}_{\alpha}^{*}(\mathscr{T})$ is compatible with a unique isomorphism

$$
\varphi_{\alpha}: L_{\alpha} \stackrel{\sim}{\longrightarrow} T_{x_{\alpha}}^{*}\left(L_{\alpha}\right)
$$

and we obtain a homomorphism $q_{\alpha}: \mathscr{G}_{\alpha}^{*}(\mathscr{T}) \rightarrow \mathscr{G}\left(L_{\alpha}\right)$ just as before. Let $K(\alpha)$ be its kernel: a subgroup of $\mathscr{G}(\mathscr{T})$ isomorphic under $\pi$ with $T(\alpha)$. 
Lemma 4. If $L_{\alpha}$ is a symmetric sheaf on $X_{\alpha}$ compatible with our polarization as above, then $K(\alpha)$ is the set of elements

$$
e_{*}^{L_{\alpha}}\left(p_{\alpha}\left(\frac{1}{2} x\right)\right) \cdot \sigma(x)
$$

$x \in T(\alpha)$. In particular, if $L_{\alpha}$ is totally symmetric, then $K(\alpha)=\sigma[T(\alpha)]$.

Proof. To unwind the definition of $\sigma(x)$, let $\beta>\alpha$ be the element of $S$ giving us a diagram:

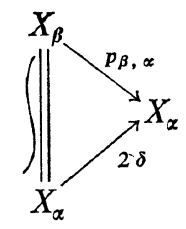

Then $p_{\beta, \alpha}^{*} L_{\alpha}$ is totally symmetric, hence isomorphic to $L_{\beta}$, and $u=p_{\beta}\left(\frac{1}{2} x\right)$ is in $H\left(L_{\beta}\right)$. Choose an isomorphism

$$
\psi: L_{\beta} \stackrel{\sim}{\longrightarrow} T_{u}^{*} L_{\beta}
$$

such that $\delta_{-1}((u, \psi))=(u, \psi)^{-1}$. Let $(u, \psi)^{2}=\left(2 u, \varphi_{\beta}\right)$. Then $\sigma(x)$ is represented by the elements $\left(2 u, \varphi_{\alpha}\right) \in \mathscr{G}\left(L_{\alpha}\right)$. Since $x \in T(\alpha), 2 u \in \operatorname{Ker}\left(p_{\beta, \alpha}\right)$ and there is a scalar $\lambda$ such that:

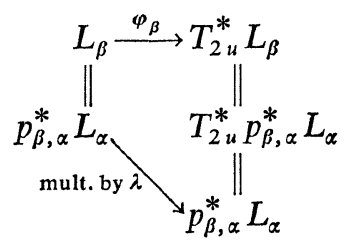

commutes. This means that $\lambda^{-1} \cdot \sigma(x)$ is compatible with the identity map from $L_{\alpha}$ to $L_{\alpha}$, i.e., is in the kernel of $q_{\alpha}$. The lemma boils down then to checking that $\lambda=e_{*}^{L_{\alpha}}\left(p_{\beta, \alpha}(u)\right)$. Throwing out irrelevant notation, we can restate this fact as:

Lemma 5. Let $L$ be a symmetric invertible sheaf of separable type on an abelian variety $X$. Let $u \in X_{4}$ and let $(u, \varphi) \in \mathscr{G}((2 \delta) * L)$ satisfy $\delta_{-1}((u, \varphi))$ $=(u, \varphi)^{-1}$. Let $(u, \varphi)^{2}=(2 u, \psi)$. Then the composite isomorphism:

$$
(2 \delta)^{*} L \stackrel{\psi}{\longrightarrow} T_{2 u}^{*}(2 \delta)^{*} L \cong(2 \delta)^{*} T_{4 u}^{*} L=(2 \delta)^{*} L
$$

is multiplication by $e_{*}^{L}(2 u)$.

Proof. Since $L$ is symmetric, $(2 \delta)^{*} L \cong L^{4}$. Let $v=2 u$ and let $(v, \rho)$ be the element $\eta_{2}((u, \varphi))$ in $\mathscr{G}\left(L^{2}\right)$. Since $\eta_{2} \circ \delta_{-1}=\delta_{-1} \circ \eta_{2}, \delta_{-1}((v, \rho))=$ $(v, \rho)^{-1}$. But $e_{*}^{L^{2}}(v)=\left[e_{*}^{L}(v)\right]^{2}=1$, so by Prop. $3, \S 2, \delta_{-1}((v, \rho))=(v, \rho)$.

8 Inventiones math., Vol. 3 
Therefore $(v, \rho)^{2}=1$ in $\mathscr{G}\left(L^{2}\right)$. Therefore by Prop. 6, $\S 2, \eta_{2}((v, \rho))=$ $\left(0, e_{*}^{L}(v)\right)$ in $\mathscr{G}(L)$. Explicitly, this means that:

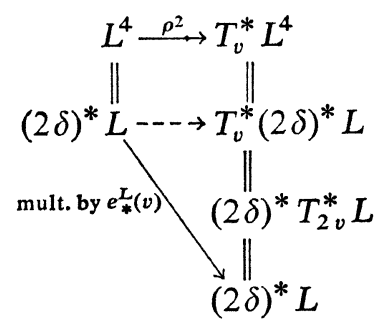

commutes. But $\left(v, \rho^{2}\right)$, as an element of $\mathscr{G}\left(L^{4}\right)$, is $\varepsilon_{2}((v, \rho))$. And

$$
\varepsilon_{2}((v, \rho))=\varepsilon_{2}\left(\eta_{2}((u, \varphi))\right)=\delta_{2}((u, \varphi))=(u, \varphi)^{3} \cdot \delta_{-1}((u, \varphi))=(v, \psi) .
$$

Therefore the dotted arrow is $\psi$ and the lemma is proven. Q.E.D.

Conversely, suppose we start with any $\alpha \in S$ such that $e \equiv 1$ on $T(\alpha) \times T(\alpha)$, i.e., $T(\alpha)$ is isotropic, and try to make a subgroup of $\mathscr{G}(\mathscr{T})$ via

$$
K(\alpha)=\left\{e_{*}\left(\frac{1}{2} x\right) \cdot \sigma(x) \mid x \in T(\alpha)\right\}
$$

where $e_{*}$ is a function from $\frac{1}{2} T(\alpha)$ to $\{ \pm 1\}$. This works if we take for $e_{*}$ any function satisfying

$$
e_{*}(x+y)=e_{\lambda}(x, y)^{2} \cdot e_{*}(x) \cdot e_{*}(y) .
$$

In particular, $e_{*}(x)=1$ if $x \in T(\alpha)$. Let $\beta \in S$ be such that $2 T(\alpha)=T(\beta)$. Then $\beta \in S_{0}$, and $K(\beta)=\sigma[T(\beta)] \subset K(\alpha)$. Let $L_{\beta}$ be the totally symmetric sheaf on $X_{\beta}$ defined by our polarized tower. Then $K(\alpha) / K(\beta)$ is a level subgroup of $\mathscr{G}\left(L_{\beta}\right)$ lying over the subgroup $p_{\beta}[T(\alpha)]$ of $H\left(L_{\beta}\right)$. As in $\S 1$, it provides descent data for $L_{\beta}$ in the isogeny $p_{\beta \alpha}: X_{\beta} \rightarrow X_{\alpha}$, since $\operatorname{Ker}\left(p_{\beta \alpha}\right)=p_{\beta}[T(\alpha)]$. Let it define $L_{\alpha}$ on $X_{\alpha}$. This $L_{\alpha}$ is easily seen to be symmetric, compatible with the polarization, and satisfying

$$
e_{*}^{L_{\alpha}}\left(p_{\alpha}(x)\right)=e_{*}(x), \quad \text { all } x \in \frac{1}{2} T(\alpha) .
$$

Conclusion. Symmetric sheaves $L_{\alpha}$ on some $X_{\alpha}$, compatible with the polarization, are in $1-1$ correspondence with all possible "level" subgroups $K \subset \mathscr{G}(\mathscr{T})$ such that

1) $K \cap k^{*}=\{1\}$,

2) for all $x \in K, \sigma(\pi(x))= \pm x$.

This ends our discussion of the groups that are involved in a polarized tower of abelian varieties. Next, we turn to their representations. The family of vector spaces

$$
\left\{\Gamma\left(X_{\alpha}, L_{\alpha}\right)\right\}, \quad \alpha \in S_{0}
$$


forms a direct system, and we define:

$$
\Gamma(\mathscr{T})=\varliminf_{\alpha \in S_{0}} \Gamma\left(X_{\alpha}, L_{\alpha}\right) .
$$

Just as in $\S 1, \mathscr{G}(\mathscr{T})$ is represented on the vector space $\Gamma(\mathscr{T})$. Moreover, as in $\S 1$, we check that we can recover $\Gamma\left(X_{\alpha}, L_{\alpha}\right)$ from $\Gamma(\mathscr{T})$ since:

$$
\Gamma\left(X_{\alpha}, L_{\alpha}\right) \cong\left\{\begin{array}{l}
\text { elements of } \Gamma(\mathscr{T}) \\
\text { invariant under } K(\alpha)
\end{array}\right\} .
$$

[The same holds for any symmetric $L_{\alpha}$ compatible with the polarization and the corresponding $K(\alpha)$.] What representations does a group like $\mathscr{G}(\mathscr{T})$ have? We make the following restriction:

Definition 9. An admissible representation $\alpha \mapsto U_{\alpha}$ of $\mathscr{G}(\mathscr{T})$ [resp. $\mathscr{G}_{g}$ ] in a vector space $V$ is one in which the subgroup $k^{*}$ acts via its natural character (i.e., if $\alpha \in k^{*}, U_{\alpha}=\alpha \cdot(i d)_{V}$ ), and such that for all $x \in V$ :

$$
\left\{\sigma \in \mathscr{G}(\mathscr{T}) \text {, resp. } \mathscr{G}_{\mathrm{g}} \mid U_{\sigma} x=\text { multiple of } x\right\},
$$

is the inverse image in $\mathscr{G}(\mathscr{T})$ [resp. $\mathscr{G}_{\mathrm{g}}$ ] of an open subset of $V(\underline{X})$ [resp. $\boldsymbol{Q}_{2}^{2 \mathrm{~g}}$ ].

Prop. 3 of $\S 1$ generalizes easily to:

Theorem. $\mathscr{G}_{\mathrm{g}}$, and hence $\mathscr{G}(\mathscr{T})$, has one and only one irreducible admissible representation. All other admissible representations break up into direct sums of the irreducible one with itself.

The proof of this is roughly as follows: choose a maximal open subgroup $U \subset Q_{2}^{28}$ on which the skew-symmetric form

$$
e\left(\left(x_{1} ; y_{1}\right),\left(x_{2} ; y_{2}\right)\right)=\chi\left[{ }^{t} x_{1} \cdot y_{2}-{ }^{t} x_{2} \cdot y_{1}\right]
$$

vanishes identically, such as $Z^{2 g}$. Then construct eigenvectors in $V$ for the subgroup $k^{*} \times U$ of $\mathscr{G}_{g}$. All other elements in the group permute these eigenvectors and we show that this permutation can be described simply, (independent of $V$ ). We leave the details to the reader as they are similar to those in the proof of Prop. 3, $\S 1$.

This irreducible representation can be written down like this:

Let

$$
\begin{gathered}
\mathscr{H}_{\mathrm{g}}=\left\{\begin{array}{l}
\text { vector space of } k \text {-valued, locally constant } \\
\text { functions } f \text { on } Q_{2}^{g}, \text { with compact support }
\end{array}\right\} . \\
{\left[U_{(\alpha, x, y)} f\right](z)=\alpha \cdot \chi\left({ }^{t} y \cdot z\right) \cdot f(x+z)} \\
\text { if } f \in \mathscr{H}_{\mathrm{g}},(\alpha, x, y) \in \mathscr{G}_{\mathrm{g}}, z \in \boldsymbol{Q}_{2}^{g} .
\end{gathered}
$$


Notice that the only elements $f \in \mathscr{H}_{\mathrm{g}}$ invariant under the operations $U_{(1, x, y)}$, all $x, y \in Z_{2}^{\mathrm{g}}$, are the multiples of the characteristic function:

$$
D(z)= \begin{cases}0, & z \notin \boldsymbol{Z}_{2}^{\mathrm{g}} \\ 1, & z \in \boldsymbol{Z}_{2}^{\mathrm{g}} .\end{cases}
$$

Therefore $\mathscr{H}_{\mathrm{g}}$ is irreducible (i.e., in view of the Theorem, if any subspace canonically attached to the representation is one-dimensional, the representation must be irreducible).

On the other hand, we have:

Theorem. $\Gamma(\mathscr{T})$ is an irreducible admissible representation for $\mathscr{G}(\mathscr{T})$.

Proof. It is an admissible representation since every $x \in \Gamma(\mathscr{T})$ is in some $\Gamma\left(X_{\alpha}, L_{\alpha}\right)$, hence is an eigenvector for $k^{*} \cdot K(\alpha) . H$ is irreducible since if $K(\alpha) \subset \mathscr{G}(\mathscr{T})$ corresponds to a symmetric $L_{\alpha}$ on $X_{\alpha}$ of degree 1 , then the subspace of $K(\alpha)$-invariants is isomorphic to $\Gamma\left(X_{\alpha}, L_{\alpha}\right)$ and this is 1-dimensional. Q.E.D.

It follows that if we choose a $\vartheta$-structure $c: \mathscr{G}(\mathscr{T}) \rightarrow \mathscr{G}_{\mathrm{g}}$, we get a unique isomorphism:

$$
\Gamma(\mathscr{T}) \stackrel{\beta}{\sim} \mathscr{H}_{\mathrm{g}}
$$

such that $\beta\left(U_{z}(s)\right)=U_{c(z)}(\beta(s))$, all $z \in \mathscr{G}(\mathscr{T}), s \in \Gamma(\mathscr{T})$. The isomorphisms $\beta$ extend the isomorphisms $\beta$ in $\S 1$ in the following way:

a. let $\alpha \in S_{0}$ and let $T(\alpha) \subset V(X)$ be a compact open subgroup such that $\bar{c}(T(\alpha))$ in $Q_{2}^{g} \times Q_{2}^{g}$ is of the form $U \times V$.

b. Then $p_{\alpha}^{-1}\left(H\left(L_{\alpha}\right)\right)$ is the orthogonal subgroup, i.e., $V^{\perp} \times U^{\perp}$ (if $V^{\perp}=\left\{x \in Q_{2}^{g} \mid \chi\left({ }^{t} x \cdot y\right)=1\right.$, all $\left.y \in V\right\}, U^{\perp}$ similar). Therefore $\bar{c}$ induces isomorphisms:

$$
H\left(L_{\alpha}\right) \cong \frac{p_{\alpha}^{-1}\left(H\left(L_{\alpha}\right)\right)}{T(\alpha)} \cong\left(V^{\perp} / U\right) \times\left(U^{\perp} / V\right) .
$$

If we choose an isomorphism

then this gives

$$
\left(V^{\perp} / U\right) \stackrel{\sim}{\longrightarrow} K(\delta),
$$

hence

$$
\left(U^{\perp} / V\right) \cong\left(\widehat{V^{\perp} / U}\right) \cong \widehat{K(\delta)},
$$

$$
H\left(L_{\alpha}\right) \cong K(\delta) \times \widehat{K(\delta)}
$$


c. Thus we get an isomorphism:

$$
\begin{aligned}
\mathscr{G}\left(L_{\alpha}\right) & \cong \frac{\mathscr{G}_{\alpha}^{*}}{K(\alpha)} \\
& \cong k^{*} \times\left(V^{\perp} / U\right) \times\left(U^{\perp} / V\right) \\
& \cong k^{*} \times K(\delta) \times \widehat{K(\delta)}=\mathscr{G}(\delta) .
\end{aligned}
$$

d. On the other hand, $\beta$ restricts as follows:

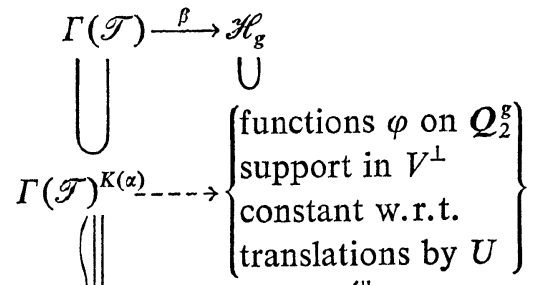

$$
\begin{aligned}
& \text { SII } \\
& \left.\Gamma\left(X_{\alpha}, L_{\alpha}\right) \quad \text { functions on } V^{\perp} / U\right\} \\
& \text { SII } \\
& \text { \{functions on } K(\delta)\}=V(\delta) \text {. }
\end{aligned}
$$

The induced isomorphism of $\Gamma\left(X_{\alpha}, L_{\alpha}\right)$ and $V(\delta)$ is exactly the isomorphism $\beta$ of $\S 1$ corresponding to the $\vartheta$-structure on $\mathscr{G}\left(L_{\alpha}\right)$ occurring in $c$.

In short, $\beta$ is just the union of the isomorphisms $\beta$ obtained on a finite level previously.

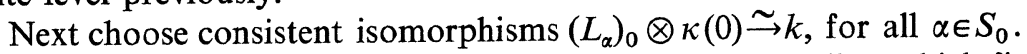
These induce "evaluation at 0 " maps: $\Gamma\left(X_{\alpha}, L_{\alpha}\right) \rightarrow k$, for all $\alpha$, which fit together into one big "evaluation at 0 " map:

$$
\Lambda: \Gamma(\mathscr{T}) \rightarrow k .
$$

To describe this piece of information in closed form, we need to describe the dual space $\mathscr{H}_{\mathrm{g}}^{*}$ :

Dual of $\mathscr{H}_{\mathrm{g}}: \mathscr{H}_{\mathrm{g}}$ is spanned by the characteristic functions $\varphi_{U}$ of compact, open subsets $U \subset \boldsymbol{Q}_{g}^{2}$, with the obvious relations:

$$
\varphi_{U_{1} \cup U_{2}}+\varphi_{U_{1} \cap U_{2}}=\varphi_{U_{1}}+\varphi_{U_{2}} .
$$

Now let $\mathscr{B}$ be the Boolean algebra of compact open subsets of $\boldsymbol{Q}_{2}^{\mathrm{g}}$. Then a linear functional on $\mathscr{H}_{\mathrm{g}}$ is determined by its values on the $\varphi_{v}$ 's, and relations which follow from (*) make this set function into a measure. In other words, if we let

$$
\mathscr{H}_{\mathrm{g}}^{*}=\left\{\begin{array}{l}
\text { vector space of } k \text {-valued finitely additive } \\
\text { measures } \mu \text { defined on the Boolean algebra } \mathscr{B}
\end{array}\right\}
$$


then the pairing:

makes $\mathscr{H}_{\mathrm{g}}^{*}$ into the dual of $\mathscr{H}_{\mathrm{g}}$.

$$
\langle f, \mu\rangle=\int_{\mathbf{Q}_{2}^{\mathrm{g}}} f \cdot d \mu
$$

This shows that there is a unique measure $\mu \in \mathscr{H}_{\mathrm{g}}^{*}$ such that

$$
\Lambda(s)=\int_{\mathbf{Q}_{2}^{\mathrm{R}}} \beta(s) \cdot d \mu
$$

all $s \in \Gamma(\mathscr{T})$. This $\mu$ is "built up" out of null functions $q_{L_{\alpha}}$ in the following way: let $\alpha \in S_{0}$ be such that $\bar{c}(T(\alpha))=U \times V$, as above. Then for all $s \in \Gamma\left(X_{\alpha}, L_{\alpha}\right), \beta(s)$ is a function on $Q_{2}^{g}$ with support in $V^{\perp}$, constant on cosets of $U$. Then

while

$$
\begin{aligned}
\Lambda(s) & =\int_{\mathbf{Q}_{2}^{\mathrm{g}}} \beta(s) \cdot d \mu \quad \text { via new theory } \\
& =\sum_{x \in V^{\perp} / U} \beta(s)(x) \cdot \mu(x+U)
\end{aligned}
$$

$$
\Lambda(s)=\sum_{x \in K(\delta)} \beta(s)(x) \cdot q_{L_{\alpha}}(x) \quad \text { via old theory. }
$$

Thus identifying $K(\delta)$ with $V^{\perp} / U$, we find:

$$
q_{L_{\alpha}}(x \bmod U)=\mu(x+U), \quad \text { all } x \in V^{\perp} .
$$

There are formulae for the other $q_{L_{\alpha}}$ 's, but they are much more complicated.

Note that since the $q_{L_{\alpha}}$ 's are known to be even functions, this formula implies that $\mu$ is an even measure on $Q_{2}^{g}$.

\section{§ 8. 2-Adic Theta Functions}

The basic idea of theta functions is to trivialize an ample sheaf $L$ on an abelian variety $X$, after pulling it back to some auxiliary space $V$ via a map $\pi: V \rightarrow X$; then sections of $L$ on $X$ become actual $k$-valued functions on $V$. In our case, let $\mathscr{T}=\left\{X_{\alpha}, L_{\alpha}\right\}$ be a polarized 2-tower of abelian varieties, and take $V=V(\underline{X})$. First, what is the "pull-back" of the $L_{\alpha}$ to $V$ ?

Let $x=\left\{x_{\alpha}\right\} \in V(\underline{X})$. Then for all $\alpha \in S$, let $L_{\alpha}\left(x_{\alpha}\right)=\left(L_{\alpha}\right)_{x_{\alpha}} \otimes_{\underline{o x}} \cdot \kappa\left(x_{\alpha}\right)$ as usual. For all $\alpha \geqq \beta, p_{\alpha \beta}^{*}$ induces an isomorphism

$$
L_{\beta}\left(x_{\beta}\right) \underset{p_{\alpha \beta}^{*}}{\sim} L_{\alpha}\left(x_{\alpha}\right) \text {. }
$$

Passing to the limit, let $L(x)$ denote the vector space you get which is canonically isomorphic to all $L_{\alpha}\left(x_{\alpha}\right)$ 's. The collection $\{L(x)\}$ of 1-dimen- 
sional vector spaces represents "the sheaf induced on $V(\underline{X})$ by the $L_{\alpha}$ 's". In particular, every $s \in \Gamma(\mathscr{T})$ has "values" $s(x) \in L(x)$ for each $x \in V(X)$.

Now let $z=\left(x,\left\{\varphi_{\alpha}\right\}\right) \in \mathscr{G}(\mathscr{T})$. For all $\alpha$ for which $\varphi_{\alpha}$ is defined, it induces an isomorphism

$$
\varphi_{\alpha}\left(x_{\alpha}\right): L_{\alpha}(y) \stackrel{\sim}{\longrightarrow} T_{x_{\alpha}}^{*}\left(L_{\alpha}\right)(y)=L_{\alpha}\left(y+x_{\alpha}\right),
$$

all $y \in X_{\alpha}$. In particular, $z$ defines an isomorphism $z_{y}: L(y) \sim(\sim) L(x+y)$ for all $y \in V(\underline{X})$. Choose as before an isomorphism $\lambda_{0}$ of $L(0)$ with $k$.

Define $\lambda_{y}$ to be the composite:

$$
L(y) \underset{\sigma(-y)_{y}}{\stackrel{\sim}{\sim}} L(0) \underset{\lambda_{0}}{\sim} k .
$$

Then $\left\{\lambda_{y}\right\}$ is a "trivialization" of $L_{\alpha}$ pulled back to $V(\underset{\exists}{X})$. In particular, if $s \in \Gamma(\mathscr{T})$, then $\lambda_{y}(s(y))$ is the value of $s$ at $y$. Define:

$$
\vartheta_{[s]}(y)=\lambda_{y}(s(y)) \text {. }
$$

This is the algebraic theta function associated to s. Alternatively, it can be expressed as:

Property I.

$$
\vartheta_{[s]}(y)=\Lambda\left[U_{\sigma(-y)}(s)\right]
$$

$$
\vartheta_{\left[U_{w}(s)\right]}(y)=\alpha \cdot e_{\lambda}(x / 2, y) \cdot \vartheta_{[s]}(y-x), \quad \text { if } w=\alpha \cdot \sigma(x) .
$$

Proof.

$$
\begin{aligned}
\vartheta_{\left[U_{w}(s)\right]}(y) & =\Lambda\left[U_{\sigma(-y)}\left(U_{w}(s)\right)\right] \\
& =\alpha \cdot \Lambda\left[U_{\sigma(-y) \cdot \sigma(x)}(s)\right] \\
& =\alpha \cdot e_{\lambda}(x / 2, y) \cdot \Lambda\left[U_{\sigma(-y+x)}(s)\right] \\
& =\alpha \cdot e_{\lambda}(x / 2, y) \vartheta_{[s]}(y-x) .
\end{aligned}
$$

Corollary. $\vartheta_{[s]}$ is a locally constant function on $V(\underline{X})$. In fact, if $s \in \Gamma\left(X_{\alpha}, L_{\alpha}\right)$, and $L_{\alpha}$ is symmetric associated to $e_{*}: \frac{1}{2} T(\alpha) \rightarrow\{ \pm 1\}$, then $\vartheta_{[s]}(y)=e *(x / 2) e_{\lambda}(x / 2, y) \cdot \vartheta_{[s]}(y-x)$. for all $x \in T(\alpha)$.

Proof. Use $U_{w}(s)=s$, whenever $w=e_{*}(x / 2) \cdot \sigma(x), x \in T(\alpha)$.

Property II. If $s_{1}, \ldots, s_{n} \in \Gamma\left(X_{\alpha}, L_{\alpha}\right)$, and $p\left(X_{1}, \ldots, X_{n}\right) \in k\left[X_{1}, \ldots, X_{n}\right]$ is homogeneous of degree $d$, then

if and only if

$$
P\left(s_{1}, \ldots, s_{n}\right)=0 \quad \text { in } \Gamma\left(X_{\alpha}, L_{\alpha}^{d}\right)
$$

$$
P\left(\vartheta_{\left[s_{1}\right]}(y), \ldots, \vartheta_{\left[s_{n}\right]}(y)\right)=0, \quad \text { all } y \in V(\underset{\leftarrow}{X}) .
$$


Equivalently, the map

$$
\vartheta: \Gamma(\mathscr{T}) \rightarrow\left\{\begin{array}{l}
\text { vector space of locally constant } \\
k \text {-valued functions on } V(X)
\end{array}\right\}
$$

extends to an injective homomorphism:

$\vartheta: \bigoplus_{d=0}^{\infty} \varliminf_{\alpha} \Gamma\left(X_{\alpha}, L_{\alpha}^{d}\right) \rightarrow\left\{\begin{array}{l}\text { ring of locally constant } \\ k \text {-valued functions on } V(X)\end{array}\right\}$.

Proof. The fact that $\vartheta$ extends to a homomorphism, or that $P\left(s_{1}, \ldots, s_{n}\right)=0$ implies $P\left(\vartheta_{\left[s_{1}\right]}(y), \ldots, \vartheta_{\left[s_{n}\right]}(y)\right)=0$ follows from the fact that $\vartheta_{[t]}(y)$ is defined as the value of $t$ at $y$, (when $L_{\alpha}$ is suitably trivialized). The fact that $\vartheta$ is injective is equivalent to noting that no non-zero section $t \in \Gamma\left(X_{\alpha}, L_{\alpha}^{d}\right)$ can vanish on all points $\operatorname{tor}_{2}\left(X_{\alpha}\right)$; and this is clear since tor ${ }_{2}\left(X_{\alpha}\right)$ is Zariski-dense in $X_{\alpha}$. Q.E.D.

Property III. If $\sigma$ is an automorphism of the field $k$, leaving fixed $k_{0} \subset k$, and if $\left(X_{\alpha}, L_{\alpha}\right)$ is defined over $k_{0}$, then $\sigma$ acts on

1) $\Gamma\left(X_{\alpha}, L_{\alpha}\right)$

2) $V(X)$

and for all $s \in \Gamma\left(X_{\alpha}, L_{\alpha}\right), y \in V(\underset{\leftarrow}{X})$

$$
\vartheta_{[\sigma(s)]}(\sigma(y))=\sigma\left[\vartheta_{[s]}(y)\right] .
$$

(More generally, it seems reasonable to expect that $\vartheta_{[s]}$ should "be defined" - in a suitable sense - over any ring $R$ over which an $X_{\alpha}$ and $L_{\alpha}$ are given.)

The proof is straightforward.

Property IV. If $\bar{c}: V\left(\underline{X)} \stackrel{\sim}{\rightarrow} Q_{2}^{g} \times Q_{2}^{g}\right.$ is a symplectic isomorphism, the transformation $T$ in the commutative diagram:

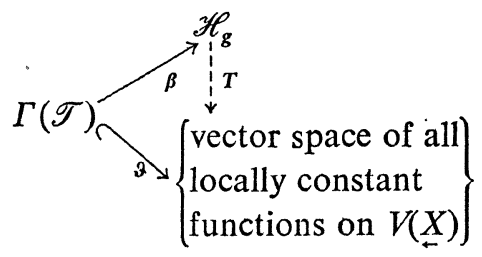

is given by:

$$
T(f)(y)=\int_{\mathbf{Q}_{2}^{\mathrm{g}}} \chi\left({ }^{t} \varphi_{2} y \cdot\left(\frac{\varphi_{1} y}{2}-u\right)\right) \cdot f\left(u-\varphi_{1} y\right) \cdot d \mu_{u}
$$

where $\bar{c}(x)=\left(\varphi_{1} x, \varphi_{2} x\right)$, and $\mu \in \mathscr{H}_{\mathrm{g}}^{*}$ is the theta-null measure of the last section. 
Proof. If $f=\beta(s)$, then

$$
\begin{aligned}
T(f)(x) & =\vartheta_{[s]}(x) \\
& =\Lambda\left(U_{\sigma(-x)}(s)\right) \\
& =\int_{\mathbf{Q}_{2}^{\mathrm{g}}}\left(U_{\Sigma\left(-\varphi_{1} x,-\varphi_{2} x\right)} f\right) \cdot d \mu \\
& =\int_{\mathbf{Q}_{2}^{\mathrm{g}}} \chi\left(\frac{{ }^{t} \varphi_{1} x \cdot \varphi_{2} x}{2}\right) \cdot \chi\left(-{ }^{t} u \cdot \varphi_{2} x\right) \cdot f\left(u-\varphi_{1} x\right) \cdot d \mu_{u} \cdot \quad \text { Q.E.D. }
\end{aligned}
$$

Corollary. A basis of $\mathscr{\vartheta}[\Gamma(\mathscr{T})]$ is given by the "classical" thetafunctions:

$$
\vartheta\left[\begin{array}{l}
a \\
b
\end{array}\right]=T\left(\delta\left[\begin{array}{l}
a \\
b
\end{array}\right]\right)
$$

where

$$
\delta\left[\begin{array}{l}
a \\
b
\end{array}\right](x)= \begin{cases}0 & \text { if } x \notin a+Z_{2}^{g} \\
\chi\left({ }^{t} b \cdot x\right) & \text { if } x \in a+Z_{2}^{g}\end{cases}
$$

and $a, b$ run through coset representatives of $Q_{2}^{g} / Z_{2}^{g}$. Here

$$
\vartheta\left[\begin{array}{l}
a \\
b
\end{array}\right](x)=\chi\left(\left(\frac{\varphi_{2} x}{2}-b\right) \cdot \varphi_{1} x\right) \cdot \int_{a+\varphi_{1}} \int_{x+\mathbf{Z}_{2}^{\mathrm{g}}} \chi\left({ }^{t}\left(b-\varphi_{2} x\right) \cdot u\right) d \mu_{u} .
$$

In particular,

$$
\vartheta\left[\begin{array}{l}
0 \\
0
\end{array}\right](x)=\chi\left(\frac{{ }^{t} \varphi_{2} x \cdot \varphi_{1} x}{2}\right) \int_{\varphi_{1}} \int_{\mathbf{Z}_{2}^{g}} \chi\left({ }^{t} \varphi_{2} x \cdot u\right) d \mu_{u}
$$

is known as RIEMANN's theta function. It is the transform via $T$ of the characteristic function $\delta\left[\begin{array}{l}0 \\ 0\end{array}\right]$ of $Z_{2}^{g}$. The multiples of $\delta\left[\begin{array}{l}0 \\ 0\end{array}\right]$ form the subspace of $\mathscr{H}_{\mathrm{g}}$ invariant under the subgroup $\{1\} \times \boldsymbol{Z}_{2}^{g} \times \boldsymbol{Z}_{2}^{g} \subset \mathscr{G}_{\mathrm{g}}$. Therefore, the multiples of $\vartheta\left[\begin{array}{l}0 \\ 0\end{array}\right]$ are exactly the elements of $\vartheta[\Gamma(\mathscr{T})]$ invariant under the subgroup

$$
K(\alpha)=c^{-1}\left(\{1\} \times Z_{2}^{g} \times Z_{2}^{g}\right) \subset \mathscr{G}(\mathscr{T}) .
$$

If $T(\alpha)$ is the compact open subgroup of $V(\underline{X})$ :

$$
T(\alpha)=\bar{c}^{-1}\left(Z_{2}^{g} \times Z_{2}^{g}\right),
$$

then in fact $K(\alpha)=\left\{e_{*}\left(\frac{1}{2} x\right) \cdot \sigma(x) \mid x \in T(\alpha)\right\}$ where

$$
e_{*}(x)=\chi\left(2^{t} \varphi_{1} x \cdot \varphi_{2} x\right), \quad \text { all } x \in \frac{1}{2} T(\alpha) \text {. }
$$

Thus $K(\alpha)$ defines a symmetric invertible sheaf $L_{\alpha}$ on the abelian variety $X_{\alpha}$, and $\Gamma\left(X_{\alpha}, L_{\alpha}\right)$ is just the space of $K(\alpha)$-invariants in $\Gamma(\mathscr{T})$. Since this 
is 1-dimensional, $L_{\alpha}$ has degree 1 , and $\vartheta\left[\begin{array}{l}0 \\ 0\end{array}\right]$ is nothing but the algebraic theta function defined by the unique section of $L_{\alpha}$ (up to scalars). Another way to put it is that

$$
\vartheta\left[\begin{array}{l}
0 \\
0
\end{array}\right] \circ \bar{c}^{-1}
$$

is the unique function $f$ of the form $\vartheta_{[s]} \circ \bar{c}^{-1}$ to satisfy the functional equation:

$$
f(x+a)=\chi\left(\frac{{ }^{t} a_{1} \cdot a_{2}}{2}\right) \cdot \chi\left(\frac{{ }^{t} a_{1} \cdot x_{2}}{2}-\frac{{ }^{t} a_{2} \cdot x_{1}}{2}\right) \cdot f(x)
$$

all $x \in Q_{2}^{2}, a \in Z_{2}^{2 g}$. Note that since $\mu$ is an even measure on $Q_{2}^{g}, \vartheta\left[\begin{array}{l}0 \\ 0\end{array}\right]$ is an even function on $V(X)$ :

$$
\vartheta\left[\begin{array}{l}
0 \\
0
\end{array}\right](-x)=\vartheta\left[\begin{array}{l}
0 \\
0
\end{array}\right](x) \text {. }
$$

An important fact is that $\mu$ can be reconstructed from $\vartheta\left[\begin{array}{l}0 \\ 0\end{array}\right]$ :

Lemma 1. There is a 1-1 correspondence between

(A.) measures $\mu \in \mathscr{H}_{\mathrm{g}}^{*}$ on $Q_{2}^{g}$,

(B.) $k$-valued functions $\vartheta$ on $Q_{2}^{g} \times Q_{2}^{g}$ such that

$$
\vartheta(x+a)=\chi\left(\frac{{ }^{t} a_{1} \cdot a_{2}}{2}\right) \cdot \chi\left(\frac{{ }^{t} a_{1} \cdot x_{2}}{2}-\frac{{ }^{t} a_{2} \cdot x_{1}}{2}\right) \cdot \vartheta(x)
$$

all $a \in Z_{2}^{g} \times Z_{2}^{g}$.

This is set up by:

$$
\begin{gathered}
\vartheta(x)=\chi\left(\frac{{ }^{t} x_{1} \cdot x_{2}}{2}\right) \cdot \int_{x_{1}+Z_{2}^{g}} \chi\left(-{ }^{t} x_{2} \cdot u\right) \cdot d \mu_{u} \\
\mu\left(a_{1}+2^{n} Z_{2}^{g}\right)=2^{-n g} \cdot \sum_{a_{2} \in 2^{-n} Z_{2}^{g} / Z_{2}^{g}} \chi\left(\frac{{ }^{t} a_{1} \cdot a_{2}}{2}\right) \cdot \vartheta\left(a_{1}, a_{2}\right) .
\end{gathered}
$$

Proof. Left to reader.

In particular, in studying the $\mu$ 's that arise from abelian varieties, it is often convenient to go back and forth between $\mu$ 's and $\vartheta$ 's that correspond as in this lemma. Let's consider RIEMANN's theta relation from this point of view. Before proving it, I want to set it up in both its $\mu$ and $\vartheta$-form:

Lemma 2. Let $\mu$ and $\vartheta$ be even measures/functions corresponding as in Lemma 1. The following conditions are equivalent: 
(A.) There is a $2^{\text {nd }}$ measure, $v \in \mathscr{H}_{\mathrm{g}}^{*}$ related to $\mu$ by the identity:

$$
\mu \times \mu(U)=v \times v(\xi(U))
$$

for all compact open subsets $U \subset Q_{2}^{g} \times Q_{2}^{g}$, where $\xi$ is the automorphism of $Q_{2}^{g} \times Q_{2}^{g}$ :

$$
\xi\left(x_{1}, x_{2}\right)=\left(x_{1}+x_{2}, x_{1}-x_{2}\right) .
$$

(B.) For all $x, y, u, v \in Q_{2}^{g} \times Q_{2}^{g}$, if $r=-\frac{1}{2}(x+y+u+v)$,

$$
\begin{aligned}
& \vartheta(x) \cdot \vartheta(y) \cdot \vartheta(u) \cdot \vartheta(v) \\
& =2^{-\mathrm{g}} . \sum_{\eta \in \frac{1}{2}} \sum_{z_{2}^{2} z / Z z_{2} g} \chi\left({ }^{t} r_{1} \cdot \eta_{2}-{ }^{t} \eta_{1} \cdot r_{2}\right) \cdot \vartheta(x+r+\eta) \times \\
& \times \vartheta(y+r+\eta) \cdot \vartheta(u+r+\eta) \cdot \vartheta(v+r+\eta) .
\end{aligned}
$$

Proof. To analyze (A.), note first that it is equivalent to the existence of a measure $v$ such that

$$
\begin{array}{r}
\int_{\mathbf{Q}_{2}^{\mathbf{g}} \times \mathbf{Q}_{2}^{\mathrm{g}}} \xi^{*}\left(\delta\left[\begin{array}{l}
2 x_{1} \\
-x_{2}
\end{array}\right] \times \delta\left[\begin{array}{l}
2 y_{1} \\
-y_{2}
\end{array}\right]\right) d \mu \times d \mu \\
=\int_{\mathbf{Q}_{2}^{\mathrm{g}} \times \mathbf{Q}_{2}^{\mathrm{g}}} \delta\left[\begin{array}{c}
2 x_{1} \\
-x_{2}
\end{array}\right] \times \delta\left[\begin{array}{l}
2 y_{1} \\
-y_{2}
\end{array}\right] d \nu \times d v
\end{array}
$$

for all $x_{1}, y_{1}, x_{2}, y_{2} \in Q_{2}^{g}$. (Here $\xi^{*}$ denotes pull-back of functions.) This is because the functions

$$
\delta\left[\begin{array}{l}
a \\
b
\end{array}\right] \times \delta\left[\begin{array}{l}
c \\
d
\end{array}\right]
$$

span the vector space of locally constant function on $\boldsymbol{Q}_{2}^{2 g}$ with compact support. But

$$
\begin{array}{r}
\xi^{*}\left(\delta\left[\begin{array}{l}
2 x_{1} \\
-x_{2}
\end{array}\right] \times \delta\left[\begin{array}{l}
2 y_{1} \\
-y_{2}
\end{array}\right]\right)(u, v)=0 \quad \text { if } u+v \notin 2 x_{1}+Z_{2}^{\mathrm{g}} \\
\text { or if } u-v \notin 2 y_{1}+Z_{2}^{\mathrm{g}} \\
=\chi\left(-{ }^{t} x_{2} \cdot(u+v)\right) \cdot \chi\left(-{ }^{t} y_{2} \cdot(u-v)\right) \\
\text { if } u \in x_{1}+y_{1}+\eta+Z_{2}^{\mathrm{g}} \\
v \in x_{1}-y_{1}+\eta+Z_{2}^{\mathrm{g}}
\end{array}
$$

for some $\eta \in \frac{1}{2} Z_{2}^{g}$.

Thus

$$
\xi^{*}\left(\delta\left[\begin{array}{c}
2 x_{1} \\
-x_{2}
\end{array}\right] \times \delta\left[\begin{array}{c}
2 y_{1} \\
-y_{2}
\end{array}\right]\right)=\sum_{\eta \in \frac{1}{2} \mathbf{Z}_{2}^{z} / \mathbf{Z}_{2}^{z}} \delta\left[\begin{array}{c}
x_{1}+y_{1}+\eta \\
-x_{2}-y_{2}
\end{array}\right] \times \delta\left[\begin{array}{c}
x_{1}-y_{1}+\eta \\
-x_{2}+y_{2}
\end{array}\right] .
$$


But note that

Given $v$, define

$$
\begin{aligned}
\int_{\mathbf{Q}_{2}^{\mathrm{g}}} \delta\left[\begin{array}{c}
x_{1} \\
-x_{2}
\end{array}\right] d \mu & =\int_{x_{1}+\mathbf{Z}_{2}^{\mathrm{g}}} \chi\left(-{ }^{t} x_{2} \cdot u\right) d \mu_{u} \\
& =\chi\left(-\frac{{ }^{t} x_{1} \cdot x_{2}}{2}\right) \cdot \vartheta\left(x_{1}, x_{2}\right)
\end{aligned}
$$

Then equation (*) is the same as:

(**) $\sum_{\eta \in \frac{1}{2} \mathbf{Z}_{2}^{\boldsymbol{z}} / \mathbf{Z}_{2}^{\mathrm{z}}} \chi\left(-{ }^{t} \eta \cdot x_{2}\right) \vartheta\left(x_{1}+y_{1}+\eta, x_{2}+y_{2}\right) \vartheta\left(x_{1}-y_{1}+\eta, x_{2}-y_{2}\right)$

$$
=\Phi\left(x_{1}, x_{2}\right) \cdot \Phi\left(y_{1}, y_{2}\right) \text {. }
$$

The reader can check that if there is a function $\Phi$ satisfying $(* *)$, this $\Phi$ comes from a $v$ satisfying (*). Thus (A.) is equivalent to the existence of a $\Phi$ satisfying (**). If we let $x=\left(x_{1}, x_{2}\right), y=\left(y_{1}, y_{2}\right), \zeta_{1}=(\eta, 0)$, then (**) becomes:

$(* *)^{\prime} \quad \sum_{\zeta_{1} \in \frac{1}{2}\left(\mathbf{Z}_{2}^{g} \times 0\right) / \mathbf{z}_{2} \times 0} \chi\left(-\zeta_{1} \cdot x_{2}\right) \vartheta\left(x+y+\zeta_{1}\right) \vartheta\left(x-y+\zeta_{1}\right)=\Phi(x) \cdot \Phi(y)$.

The existence of a $\Phi$ satisfying this is clearly equivalent to:

$(* * *)$

$$
\begin{aligned}
& \sum_{\zeta_{1}} \chi\left(-\zeta_{1} \cdot x_{2}\right) \vartheta\left(x+y+\zeta_{1}\right) \vartheta\left(x-y+\zeta_{1}\right) \times \\
& \times \sum_{\zeta_{1}} \chi\left(-\zeta_{1} \cdot u_{2}\right) \vartheta\left(u+v+\zeta_{1}\right) \vartheta\left(u-v+\zeta_{1}\right) \\
&= \sum_{\zeta_{1}} \chi\left(-\zeta_{1} \cdot x_{2}\right) \vartheta\left(x+v+\zeta_{1}\right) \vartheta\left(x-v+\zeta_{1}\right) \times \\
& \times \sum_{\zeta_{1}} \chi\left(-\zeta_{1} \cdot u_{2}\right) \vartheta\left(u+y+\zeta_{1}\right) \vartheta\left(u-y+\zeta_{1}\right)
\end{aligned}
$$

for all $x, y, u, v \in \boldsymbol{Q}_{2}^{2 g}$ (the summations being as in $\left.(* *)^{\prime}\right)$.

In $(* * *)$, replace $x$ by $x+\zeta_{2}, y$ by $y+\zeta_{2}, u$ by $u+\zeta_{2}^{\prime}$ and $v$ by $v+\zeta_{2}^{\prime}$, where $\zeta_{2}, \zeta_{2}^{\prime} \in 0 \times\left(\frac{1}{2} Z_{2}^{g}\right)$. Multiply by $\chi\left({ }^{t} \zeta_{2} \cdot x_{1}+y_{1}\right) \cdot \chi\left({ }^{t} \zeta_{2}^{\prime} \cdot u_{1}+v_{1}\right)$ and sum over all $\zeta_{2}, \zeta_{2}^{\prime} \bmod 0 \times \boldsymbol{Z}_{2}^{\mathrm{g}}$. Then out comes $(B) !$ Reversing this, you can get $(* * *)$ out of $(B)$. Q.E.D.

Definition. Even measures $\mu$ with property (A.) above will be called Gaussian.

We now intend to prove that if

$$
\vartheta=\vartheta\left[\begin{array}{l}
0 \\
0
\end{array}\right]
$$

and $\mu$ is the null-value measure, arising from a polarized tower $\mathscr{T}$, plus $\vartheta$-structure $c$, then these conditions on $\mu$ and $\vartheta$ hold. This fact is derived 
by comparing

with the new tower

$$
\mathscr{T}=\left\{X_{\alpha}, L_{\alpha}\right\}
$$

$$
\mathscr{T}^{(2)}=\left\{X_{\alpha}, L_{\alpha}^{2}\right\} \text {. }
$$

Notice, incidentally, that the relationship between the towers $\mathscr{T}$ and $\mathscr{T}^{(2)}$ is symmetric, in that if we let

$$
\mathscr{T}^{(4)}=\left\{X_{\alpha}, L_{\alpha}^{4}\right\}
$$

then $\mathscr{T}^{(4)}$ is isomorphic to $\mathscr{T}$ again. In fact, for all $\alpha \in S$, let $2 * \alpha$ be the new index such that $2 * \alpha>\alpha$ and such that we get a diagram:

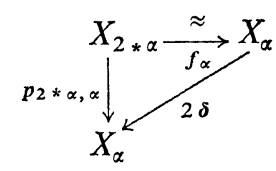

Putting together all these isomorphisms, we find

so

$$
\begin{gathered}
L_{2 \star \alpha} \cong f_{\alpha}^{*}\left(L_{\alpha}^{4}\right), \text { hence }\left(X_{2 \star \alpha}, L_{2 * \alpha}\right) \cong\left(X_{\alpha}, L_{\alpha}^{4}\right) \\
\mathscr{T}=\left\{X_{2 \star \alpha}, L_{2 \star \alpha}\right\} \cong\left\{X_{\alpha}, L_{\alpha}^{4}\right\}=\mathscr{T}^{(4)} .
\end{gathered}
$$

The natural homomorphisms $\varepsilon_{2}$ and $\eta_{2}$ can be defined as in $\S 2$, and we get a diagram:

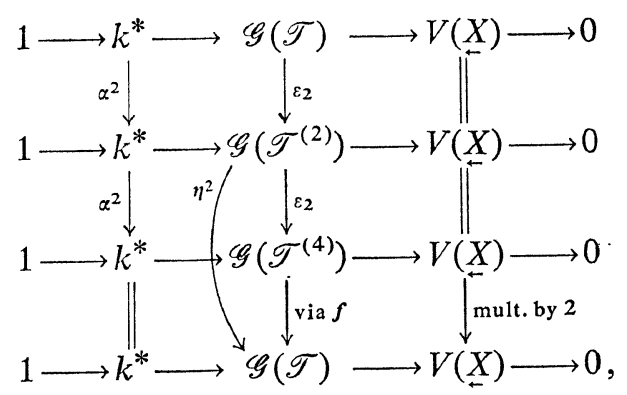

and everything commutes with $\delta_{-1}$ and $\sigma$. In terms of our standard groups, this diagram goes over to:

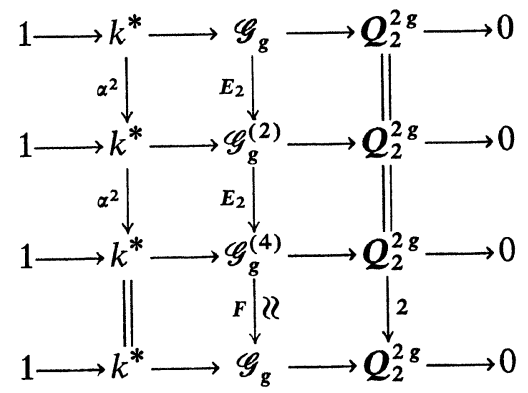


where $\mathscr{G}_{\mathbf{g}}^{(2)}$ and $\mathscr{G}_{\mathbf{g}}^{(4)}$ are both equal to $k^{*} \times Q_{2}^{g} \times Q_{2}^{g}$, but with group laws:

resp.

$$
(\alpha, x, y) \cdot\left(\alpha^{\prime}, x^{\prime}, y^{\prime}\right)=\left(\alpha \cdot \alpha^{\prime} \cdot \chi\left(2^{t} x \cdot y^{\prime}\right), x+x^{\prime}, y+y^{\prime}\right)
$$

and

$$
=\left(\alpha \cdot \alpha^{\prime} \cdot \chi\left(4^{t} x \cdot y^{\prime}\right), x+x^{\prime}, y+y^{\prime}\right.
$$

$$
\begin{aligned}
E_{2}(\alpha, x, y) & =\left(\alpha^{2}, x, y\right), \\
F(\alpha, x, y) & =(\alpha, 2 x, 2 y) .
\end{aligned}
$$

Thus given one symplectic isomorphism

$$
\begin{aligned}
V(\underset{X}{X}) & \rightarrow Q_{2}^{\mathrm{g}} \times Q_{2}^{g}, \\
x & \mapsto\left(\varphi_{1} x, \varphi_{2} x\right)
\end{aligned}
$$

we get symmetric theta structures

$c_{1}: \mathscr{G}(\mathscr{T}) \longrightarrow \mathscr{G}_{\mathrm{g}}, \quad c_{2}: \mathscr{G}\left(\mathscr{T}^{(2)}\right) \stackrel{\sim}{\longrightarrow} \mathscr{G}_{\mathrm{g}}^{(2)}$ and $c_{4}: \mathscr{G}\left(\mathscr{T}^{(4)}\right) \stackrel{\sim}{\longrightarrow} \mathscr{G}_{\mathrm{g}}^{(4)}$.

Via the theta function representation, we obtain injections:

$$
\begin{aligned}
\vartheta: \Gamma(\mathscr{T}) & \rightarrow\left\{\begin{array}{l}
\text { functions } \\
\text { on } V(\underset{\perp}{X})
\end{array}\right\}, \\
\vartheta^{(2)}: \Gamma\left(\mathscr{T}^{(2)}\right) & \rightarrow\left\{\begin{array}{l}
\text { functions } \\
\text { on } V(\underset{\leftarrow}{X})
\end{array}\right\}
\end{aligned}
$$

and it is easy to check that, for all $s_{1}, s_{2} \in \Gamma\left(X_{\alpha}, L_{\alpha}\right)$

$$
\vartheta^{(2)}\left(s_{1} \otimes s_{2}\right)=\vartheta\left(s_{1}\right) \cdot \vartheta\left(s_{2}\right),
$$

i.e., tensor product of sections becomes pointwise multiplication of theta functions (compare Property II above).

Now, define actions of both $\mathscr{G}(\mathscr{T})$ and $\mathscr{G}\left(\mathscr{T}^{(2)}\right)$ on the vector space of all $k$-valued functions on $V(\underline{X})$ by:

if

$$
\left(U_{w}(\varphi)\right) y=\alpha \cdot e_{\lambda}\left(\frac{x}{2}, y\right) \cdot \varphi(y-x)
$$

if

$$
\begin{gathered}
w=\alpha \cdot \sigma(x) \in \mathscr{G}(\mathscr{T}), \\
\left(U_{w}^{(2)}(\varphi)\right) y=\alpha \cdot e_{\lambda}(x, y) \cdot \varphi(y-x) \\
w=\alpha \cdot \sigma(x) \in \mathscr{G}\left(\mathscr{T}^{(2)}\right) .
\end{gathered}
$$

Then according to Property I of algebraic theta functions, Image $(\vartheta)$ is an irreducible $\mathscr{G}(\mathscr{T})$-space, and Image $\left(\vartheta^{(2)}\right)$ is an irreducible $\mathscr{G}\left(\mathscr{T}^{(2)}\right)$ space. Moreover, Image $(\vartheta)$ must be generated by the various functions

$$
U_{w}\left(\vartheta\left[\begin{array}{l}
0 \\
0
\end{array}\right]\right)
$$


i.e.,

$$
y \mapsto e_{\lambda}\left(\frac{x}{2}, y\right) \cdot \vartheta\left[\begin{array}{l}
0 \\
0
\end{array}\right](y-x)
$$

and Image $\left(\vartheta^{(2)}\right)$ must be generated by their various products:

$$
y \mapsto e_{\lambda}\left(\frac{x_{1}+x_{2}}{2}, y\right) \cdot \vartheta\left[\begin{array}{l}
0 \\
0
\end{array}\right]\left(y-x_{1}\right) \cdot \vartheta\left[\begin{array}{l}
0 \\
0
\end{array}\right]\left(y-x_{2}\right) .
$$

Now it is a non-trivial condition that this second family of functions spans an irreducible $\mathscr{G}\left(\mathscr{T}^{(2)}\right)$-space. In particular, let $K \subset \mathscr{G}\left(\mathscr{T}^{(2)}\right)$ be the subgroup:

$$
c_{2}^{-1}\left[\left\{(1, x, y) \mid x \in \frac{1}{2} Z_{2}^{\mathrm{g}}, y \in Z_{2}^{\mathrm{g}}\right\}\right] .
$$

Then in an irreducible $\mathscr{G}\left(\mathscr{T}^{(2)}\right)$-space, $K$ has a one-dimensional space of invariants. Now it is easy to check that all the functions

$$
y \mapsto \vartheta\left[\begin{array}{l}
0 \\
0
\end{array}\right](y+x) \cdot \vartheta\left[\begin{array}{l}
0 \\
0
\end{array}\right](y-x)
$$

(any $x \in V(\underset{X}{X})$ ) are invariant under $c_{2}^{-1}\left(1 \times Z_{2}^{g} \times Z_{2}^{g}\right)$. Let $V_{1} \subset V(\underset{\underline{X}}{X})$ be the subgroup $\bar{c}_{2}^{-1}\left(Z_{2}^{g} \times\{0\}\right)$. Then the functions:

$$
y \mapsto \sum_{\zeta \in \frac{1}{2} V_{1} / V_{1}} e_{\lambda}(y, \zeta) \cdot \vartheta\left[\begin{array}{l}
0 \\
0
\end{array}\right](y+x+\zeta) \cdot \vartheta\left[\begin{array}{l}
0 \\
0
\end{array}\right](y-x+\zeta)
$$

are all invariant under $K$. It follows that they are all proportional to one function $\Phi(y)$. Therefore, there are constants, depending on $x$ - call them $c(x)$ - such that

$$
\sum_{\zeta \in \frac{1}{2} V_{1} / V_{1}} e_{\lambda}(y, \zeta) \vartheta\left[\begin{array}{l}
0 \\
0
\end{array}\right](y+x+\zeta) \cdot \vartheta\left[\begin{array}{l}
0 \\
0
\end{array}\right](y-x+\zeta)=c(x) \cdot \Phi(y) .
$$

Interchanging $x$ and $y$ in this expression, using the evenness of $\vartheta\left[\begin{array}{l}0 \\ 0\end{array}\right]$ and its periodicity with respect to elements of $V_{1}$, you check that the left-hand side is symmetric in $x$ and $y$. Thus

$$
c(x) \cdot \Phi(y)=c(y) \cdot \Phi(x), \quad \text { all } x, y \in V(\underset{X}{X}) .
$$

Since neither $c$ nor $\Phi$ can be identically 0 , this implies that $c(x)=\alpha \cdot \Phi(x)$, for all $x$ and some $\alpha \in k^{*}$. Replacing $\Phi$ by $\sqrt{\alpha} \cdot \Phi$, we get $c=\Phi$, or

$$
\sum_{\zeta \in\left\{\begin{array}{l}
2 \\
V_{1} / V_{1}
\end{array}\right.} e_{\lambda}(y, \zeta) \cdot \vartheta\left[\begin{array}{l}
0 \\
0
\end{array}\right](y+x+\zeta) \cdot \vartheta\left[\begin{array}{l}
0 \\
0
\end{array}\right](y-x+\zeta)=\Phi(x) \cdot \Phi(y) .
$$

This is equation (**)' in the proof of Lemma 2, so referring to this proof, we see that we have proven that $\vartheta\left[\begin{array}{l}0 \\ 0\end{array}\right]$ satisfies RIEMANN's theta relation.

Because of the central significance of this result, I want to give a second proof, following the lines of the proof in $\S 3$ in the finite case. 
First, introduce the maps:
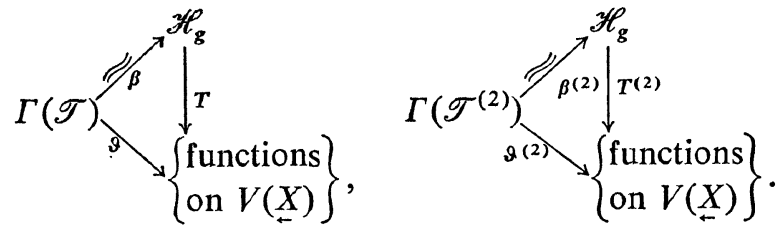

These induce a pairing

such that equivalently

$$
\circ: \mathscr{H}_{\mathrm{g}} \times \mathscr{H}_{\mathrm{g}} \rightarrow \mathscr{H}_{\mathrm{g}}
$$

or

$$
\beta\left(s_{1}\right) \circ \beta\left(s_{2}\right)=\beta^{(2)}\left(s_{1} \otimes s_{2}\right), \quad s_{i} \in \Gamma(\mathscr{T})
$$

$$
T\left(f_{1}\right) \cdot T\left(f_{2}\right)=T^{(2)}\left(f_{1} \circ f_{2}\right), \quad f_{i} \in \mathscr{H}_{g} .
$$

Notice that the algebraic tensor product $\mathscr{H}_{\mathrm{g}} \otimes \mathscr{H}_{\mathrm{g}}$ is just $\mathscr{H}_{2 \mathrm{~g}}$. In particular, the map

$$
f_{1}, f_{2} \mapsto f_{1} \circ f_{2}(0)
$$

is a linear functional on $\mathscr{H}_{\mathrm{g}} \otimes \mathscr{H}_{\mathrm{g}}$, hence it is represented by a finitely additive measure $\lambda$ on $\boldsymbol{Q}_{2}^{\mathrm{g}} \times \boldsymbol{Q}_{2}^{\mathrm{g}}$ : i.e.,

$$
f_{1} \circ f_{2}(0)=\int_{Q_{2}^{\mathrm{g}} \times \mathbf{Q}_{2}^{\mathrm{g}}} f_{1}(u) \cdot f_{2}(v) \cdot d \lambda_{u, v} .
$$

Since for all $s_{1}, s_{2} \in \Gamma(\mathscr{T}), \alpha \in \mathscr{G}(\mathscr{T}), U_{\alpha} s_{1} \otimes U_{\alpha} s_{2}=U_{\varepsilon_{2}(\alpha)}\left(s_{1} \otimes s_{2}\right)$, we find that for all $f_{1}, f_{2} \in \mathscr{H}_{g}, \alpha \in \mathscr{G}_{g}, U_{\alpha} f_{1} \circ U_{\alpha} f_{2}=U_{\varepsilon_{2}(\alpha)}\left(f_{1} \circ f_{2}\right)$. Let $\alpha=\left(1, y_{1}, y_{2}\right)$. Then:

$$
\begin{aligned}
f_{1} \circ f_{2}\left(y_{1}\right) & =\left(U_{\left(1, y_{1}, y_{2}\right)}\left(f_{1} \circ f_{2}\right)\right)(0) \\
& =\left[U_{\left(1, y_{1}, y_{2}\right)} f_{1} \circ U_{\left(1, y_{1}, y_{2}\right)} f_{2}\right](0) \\
& =\int_{\boldsymbol{Q}_{2}^{\mathrm{g}} \times \mathbf{Q}_{2}^{\mathrm{g}}} \chi\left({ }^{t} y_{2} \cdot(u+v)\right) \cdot f_{1}\left(u+y_{1}\right) \cdot f_{2}\left(v+y_{1}\right) \cdot d \lambda_{u, v}
\end{aligned}
$$

for all $y_{1}, y_{2} \in Q_{2}^{g}$. Taking combinations of these equations for various $y_{2}$ 's, it follows that

$$
\int_{\mathbf{Q}_{2}^{\mathbf{8}} \times \mathbf{Q}_{2}^{\mathrm{g}}} g(u+v) \cdot f_{1}\left(u+y_{1}\right) \cdot f_{2}\left(v+y_{2}\right) \cdot d \lambda_{u, v}=0
$$

for all locally constant functions $g$ such that $g(0)=0$. This shows that

$$
\int h(u, v) d \lambda_{u, v}=0
$$

whenever $h \in \mathscr{H}_{2 \mathrm{~g}}$, and $h(u,-u)=0$, all $u$. This implies that $\lambda_{u, v}$ is given by a measure on the set $\{(u,-u)\}$, i.e., there is a $\tilde{\lambda}_{\in} \mathscr{H}_{\mathrm{g}}{ }^{*}$ such that

$$
\int h(u, v) d \lambda_{u, v}=\int h(u,-u) d \tilde{\lambda}_{u}
$$


all $h \in \mathscr{H}_{2 g}$. Therefore:

$$
f_{1} \circ f_{2}(y)=\int_{\mathbf{Q}_{2}^{\mathrm{g}}} f_{1}(y+u) f_{2}(y-u) d \tilde{\lambda}_{u} .
$$

Now use the fact that $s_{1} \otimes s_{2}(0)=s_{1}(0) \cdot s_{2}(0)$ : therefore if $\mu$ and $v$ are the null-value measures for $\mathscr{T}$ and $\mathscr{T}^{(2)}$, we find

$$
\begin{aligned}
\int_{\mathbf{Q}_{2}^{\mathrm{g}}} f_{1}(u) d \mu_{u} \cdot \int_{\mathbf{Q}_{2}^{\mathrm{g}}} f_{2}(v) d \mu_{v} & =\int_{\mathbf{Q}_{2}^{\mathrm{g}}} f_{1} \circ f_{2}(w) d v_{w} \\
& =\int_{\mathbf{Q}_{2}^{\mathrm{g} \times \mathbf{Q}_{2}^{\mathrm{g}}}} f_{1}(w+t) f_{2}(w-t) d v_{w} \cdot d \tilde{\lambda}_{t} .
\end{aligned}
$$

Hence if $\xi(x, y)=(x+y, x-y)$ as usual, we find

i. e.,

$$
\begin{gathered}
\int_{\mathbf{Q}_{2}^{\mathrm{g}}} F \cdot d(\mu \times \mu)=\int_{\substack{\mathbf{Q}_{2}^{2} \mathrm{~g} \\
\mu \times \mu(U)=v \times \lambda\left(\xi^{-1} U\right)}} \xi^{*} F \cdot d(v \times \tilde{\lambda}) \quad \text { for all } F \in \mathscr{H}_{2 g},
\end{gathered}
$$

for all compact open sets $U \subset Q_{2}^{2 g}$. Using the evenness of $\mu$, it follows from this equation that $v \times \tilde{\lambda}=\tilde{\lambda} \times v$, hence $v$ and $\tilde{\lambda}$ are proportional. Changing $v$ by a constant, which is permissible, we may assume $v=\tilde{\lambda}$. Then if $v^{\prime}$ is the measure

$$
v^{\prime}(U)=v\left(\frac{1}{2} U\right),
$$

it follows that $\mu \times \mu(U)=v^{\prime} \times v^{\prime}(\xi U)$, all $U$. This is condition $(A)$ of Lemma 2.

\section{§9. The 2-Adic Moduli Space}

We will now put the results of $\S 8$ in a moduli-theoretic form, and relate these to the finite-level results of $\S 6$. Once we have done this, we will be able to go further and determine the structure of the boundary of the moduli space.

The whole moduli problem for abelian varieties looks very different when viewed from an isogeny invariant point of view. The difference between polarization types disappears because any abelian variety is isogenous to a principally polarized abelian variety (as is easily proven by generalizing some of the results of $\S 1$ to inseparably polarized abelian varieties). The natural thing is then to view the classification in 2 steps: first one has the totality of polarized towers; second, within each tower one has a huge system of variously polarized abelian varieties. We will not treat this entire moduli problem since the study of all inseparably polarized abelian varieties within an isogeny type is a subject in itself. In order to a) restrict to separably polarized abelian varieties, while 
b) constructing moduli schemes simultaneously in as many characteristics as possible, we shall consider only the polarized 2-tower inside each full polarized tower, and at the same time exclude only char. 2. Analogous results would be obtained if we restricted ourselves to all characteristics $p, p \nmid d$ ( $d$ a fixed even integer), and to isogenies within a tower of degree dividing $d^{N}, N \gg 0$. As far as the category of sets is concerned, we have the following sets and canonical maps to consider:

$$
\begin{gathered}
\mathscr{M}_{\infty}=\left\{\begin{array}{l}
\text { set of polarized 2-towers } \mathscr{T}=\left\{X_{\alpha}, L_{\alpha}\right\} \\
\text { plus symplectic isomorphisms } \\
\varphi: V(X) \underset{\sim}{\sim} Q_{2}^{g} \times Q_{2}^{g} \\
\text { up to isomorphism }
\end{array}\right\} \\
\mathscr{M}_{\delta_{1}}=\left\{\begin{array}{l}
\text { set of abelian varieties } X, \\
\text { ample totally symmetric sheaves } \\
L \text { of type } \delta_{1}, \text { and symmetric } \\
\text { theta-structures } \beta: \mathscr{G}(L) \stackrel{\sim}{\sim} \mathscr{G}\left(\delta_{1}\right) \\
\text { up to isomorphism }
\end{array}\right\} \\
\mathscr{M}_{\delta_{1}}^{0}=\left\{\begin{array}{l}
\text { set of abelian varieties } X, \text { plus } \\
\text { ample totally symmetric sheaves } \\
L \text { of type } \delta_{1} \text { up to isomorphism }
\end{array}\right\} \\
\mathscr{M}_{-\infty}=\left\{\begin{array}{l}
\text { set of polarized 2-towers } \\
\mathscr{T}=\left\{X_{\alpha}, L_{\alpha}\right\} \\
\text { up to isomorphism }
\end{array}\right\}
\end{gathered}
$$

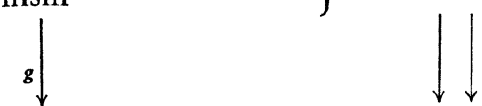

Here $\delta_{1}$ is any $g$-tuple $\left(2^{n_{1}}, 2^{n_{2}}, \ldots, 2^{n_{g}}\right), n_{1} \geqq n_{2} \geqq \cdots \geqq n_{g} \geqq 1$. The various arrows arise as follows:

(I) $g$ takes $(X, L, \beta)$ to $(X, L)$,

(II) $h$ takes $(X, L)$ to the 2-tower generated by $(X, L)$.

(III) The $f_{i}$ 's are given by choosing a compact, open isotropic subgroup $U \subset Q_{2}^{28}$ such that $U^{\perp} / U \cong H\left(\delta_{1}\right)$, and a symmetric isomorphism

$$
\beta_{0}: \frac{k^{*} \times \Sigma\left(U^{\perp}\right)}{\Sigma(U)} \stackrel{\approx}{\longrightarrow} \mathscr{G}\left(\delta_{1}\right)
$$


Then for all $(\mathscr{T}, \varphi)$, there is a unique level $\alpha \in S$ such that $\varphi(T(\alpha))=U$, and $f((\mathscr{T}, \varphi))$ should be $\left(X_{\alpha}, L_{\alpha}, \beta\right)$ where $\beta$ is the composite

$$
\mathscr{G}\left(L_{\alpha}\right) \underset{\text { via } \varphi}{\stackrel{\approx}{\longrightarrow}} \frac{k^{*} \times \Sigma\left(U^{\perp}\right)}{\Sigma(U)} \underset{\beta_{0}}{\approx} \mathscr{G}\left(\delta_{1}\right) .
$$

It is apparent from this diagram that the various moduli sets $\mathscr{M}_{\delta}$ treated in $\S 6$ are inter-related in a rather complicated way: Given $\delta_{1}, \delta_{2}$, one can choose any of an infinite number of $f_{1}, f_{2}$ in the diagram:<smiles>[Mg][Mg][Mg]</smiles>

and relate $\mathscr{M}_{\delta_{1}}, \mathscr{M}_{\delta_{2}}$ via the, in general, many-many correspondence so obtained. Each $\mathscr{M}_{\delta}$ is related to itself in this way by the well-known Hecke ring of correspondences. The whole set-up is much easier to visualize starting from $\mathscr{M}_{\infty}$. Note that $G=\operatorname{Sp}\left(2 g, Q_{2}\right)$ acts on $\mathscr{M}_{\infty}$, if we let $\sigma \in G$ act as follows:

$$
\sigma(\mathscr{T}, \varphi)=(\mathscr{T}, \sigma \circ \varphi)
$$

Then $\mathscr{M}_{-\infty}$ is nothing but the quotient $\mathscr{M}_{\infty} / G$, and the $\mathscr{M}_{\delta}$ and $\mathscr{M}_{\delta}^{0}$ 's are quotients $\mathscr{M}_{\infty} / \Gamma$ where $\Gamma \subset G$ is a suitable subgroup commensurable with $\operatorname{Sp}\left(2 g, Z_{2}\right)$. The different maps from $\mathscr{M}_{\infty}$ to $\mathscr{M}_{\delta}$ are simply the compositions of (a) action of some $\sigma \in \operatorname{Sp}\left(2 g, Q_{2}\right)$ and (b) the canonical map from $\mathscr{M}_{\infty}$ to $\mathscr{M}_{\infty} / \Gamma$. Clearly the most basic sets to get ahold of are $\mathscr{M}_{-\infty}$ and $\mathscr{M}_{\infty}$. We have seen that the $\mathscr{M}_{\delta}$ 's "are" varieties. Thus the $\mathscr{M}_{\infty}$ is an infinite covering of a variety and $\mathscr{M}_{-\infty}$ is an infinite quotient. As far as I know there is no sensible object whose underlying point set is $\mathscr{M}_{-\infty}$. But $\mathscr{M}_{\infty}$ is an inverse limit of varieties and will turn out to be a perfectly upstanding (though non-noetherian) scheme. This moduli space (and its adelic generalizations) seem to be the most important ones for the entire moduli theory of abelian varieties.

The next step is to define the scheme an open subset of which will represent $\mathscr{M}_{\infty}$. We will work over the following ground ring $R$ :

Definition 1. $R=Z\left[\frac{1}{2}, \zeta_{1}, \zeta_{2}, \zeta_{3}, \ldots\right]$ where $\zeta_{n}^{2}=\zeta_{n-1}$ if $n \geqq 2, \zeta_{1}=-1$. The multiplicative group generated by the $\zeta$ 's is isomorphic to $\boldsymbol{Q}_{2} / \boldsymbol{Z}_{2}$ and we define:

via

$$
\chi: Q_{2} / Z_{2} \rightarrow R^{*}
$$

$$
\chi\left(m / 2^{n}\right)=\left(\zeta_{n}\right)^{m}
$$

Actually, adjoining the $\zeta$ 's is not essential, but it makes life easier and seems to be quite natural.

9* 
Next, to $R$ adjoin independent transcendentals $X_{\alpha}$, one for each $\alpha \in Q_{2}^{2 g}$. Then divide out by the following relations:

1) $X_{\alpha+\beta}=\chi\left(\frac{{ }^{t} \beta_{1} \cdot \beta_{2}}{2}\right) \chi\left(\frac{{ }^{t} \beta_{1} \cdot \alpha_{2}}{2}-\frac{{ }^{t} \alpha_{1} \cdot \beta_{2}}{2}\right) \cdot X_{\alpha}$ all $\alpha \in Q_{2}^{2 g}, \beta \in Z_{2}^{2 g}$.

2) $X_{-\alpha}=X_{\alpha}$.

3) $\prod_{i=1}^{4} X_{\alpha_{i}}=2^{-g} \sum_{\eta \in \frac{1}{2}} \sum_{Z_{2} g / Z_{2}^{2} g} \chi\left({ }^{t} \gamma_{1} \cdot \eta_{2}-{ }^{t} \gamma_{2} \cdot \eta_{1}\right) \cdot \prod_{i=1}^{4} X_{\alpha_{i}+\gamma+\eta}$ all $\alpha_{1}, \alpha_{2}, \alpha_{3}, \alpha_{4} \in Q_{2}^{2 g}$, where $\gamma=-\frac{1}{2} \sum_{i=1}^{4} \alpha_{i}$.

In what follows, it will be convenient to abbreviate the characters in these formulas as follows:

Definition 2.

$$
\begin{aligned}
e(\alpha, \beta) & =\chi\left({ }^{t} \alpha_{1} \cdot \beta_{2}-{ }^{t} \beta_{1} \cdot \alpha_{2}\right), & & \alpha, \beta \in Q_{2}^{2 g} . \\
e_{*}(\alpha) & =\chi\left(2^{t} \alpha_{1} \cdot \alpha_{2}\right), & & \text { if } \alpha \in \frac{1}{2} Z_{2}^{2 g} .
\end{aligned}
$$

Definition 3.

$$
\begin{gathered}
A=R\left[\ldots, X_{\alpha}, \ldots\right] /\left\{\begin{array}{l}
\text { ideal generated by } \\
\text { relations } 1,2,3
\end{array}\right\} . \\
\bar{M}_{\infty}=\operatorname{Proj}(A) .
\end{gathered}
$$

In order to get a preliminary idea of how big $\bar{M}_{\infty}$ is, introduce the subrings:

Definition 4. $A_{n}=$ subring of $A$ generated by

$$
\left\{X_{\alpha} \mid 2^{n} \alpha \in Z_{2}^{2 g}\right\} \text {. }
$$

Lemma 1. $A$ is integrally dependent on $A_{2}$.

Proof. By induction, it suffices to check that $X_{\alpha}$ is integrally dependent on $A_{n-1}$, when $2^{n} \cdot \alpha \in Z_{2}^{2 g}$ and $n \geqq 2$. Use relation (3) with $\alpha_{1}=\alpha_{2}=\alpha_{3}=\alpha$, $\alpha_{4}=\gamma=-\alpha$. Then since $X_{-\alpha}=X_{\alpha}$, we find that $X_{\alpha}^{4} \in A_{n-1}$. Q.E.D.

Corollary. There are integral affine morphisms:

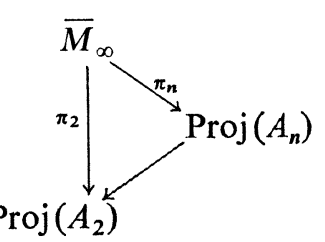


and $\bar{M}_{\infty}$ is the category-theoretic $\varliminf$ of the algebraic schemes $\operatorname{Proj}\left(A_{n}\right)$, i.e., for all schemes $S$,

$$
\operatorname{Hom}\left(S, \bar{M}_{\infty}\right) \cong \varliminf \operatorname{Hom}\left(S, \operatorname{Proj}\left(A_{n}\right)\right) .
$$

Proof. Cf. EGA, Ch. 4.

The $X_{\alpha}$ 's will be nothing but the values of the function $\vartheta\left[\begin{array}{l}0 \\ 0\end{array}\right]$ when we connect $\bar{M}_{\infty}$ to the moduli problem. It is also convenient to introduce a second set of generators of the ring $A$, whose values will be the values of the measure $\mu$, in the moduli problem:

For all compact open sets $U \subset Q_{2}^{g}$, let

if

$$
Y_{U}=2^{-n g} \sum_{i=1}^{N} \sum_{\beta \in 2^{-n} \mathbf{Z}_{2}^{\frac{\beta}{2} / \mathbf{Z}_{2}^{2}}} \chi\left(\frac{{ }^{t} \alpha_{i} \cdot \beta}{2}\right) X_{\left(\alpha_{i}, \beta\right)}
$$

$$
U=\bigcup_{i=1}^{N}\left[\alpha_{i}+2^{n} Z_{2}^{g}\right] \text { and } \alpha_{i} \neq \alpha_{j}\left(\bmod 2^{n} Z_{2}^{g}\right) \text {. }
$$

Using Lemmas 1 and $2, \S 8$, the relations on the $X$ 's go over to the following relations in the $Y$ 's:

1. $Y_{U_{1}}+Y_{U_{2}}=Y_{U_{1} \cup U_{2}}+Y_{U_{1} \cap U_{2}}$.

2. $Y_{-U}=Y_{U}, Y_{\not{g}}=0$.

3. If we define quadratic polynomials, $Z_{U}$, for all compact open sets $U \subset Q_{2}^{g} \times Q_{2}^{g}$, by relations (1.) and $Z_{U_{1} \times U_{2}}=Y_{U_{1}} Y_{U_{2}}$, and if $\xi(x, y)=$ $(x+y, x-y)$ as usual, then:

$$
Z_{\xi\left(U_{1} \times U_{2}\right)} \cdot Z_{\xi\left(U_{3} \times U_{4}\right)}=Z_{\xi\left(U_{1} \times U_{4}\right)} \cdot Z_{\xi\left(U_{3} \times U_{2}\right)} .
$$

In particular, let $n \geqq 1$ and let

$$
l: 2^{n-1} Z_{2}^{g} \rightarrow\{ \pm 1\}
$$

be a homomorphism. Define

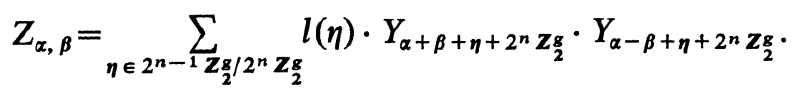

Then the general relations imply:

$$
Z_{\alpha, \beta} \cdot Z_{\gamma, \delta}=Z_{\alpha, \delta} \cdot Z_{\gamma, \beta}
$$

for all $\alpha, \beta, \gamma, \delta \in Q_{2}^{g}$. Conversely, these relations, for all $n$ 's, and $l \equiv+1$, imply all the quartic relations. 
Moreover, the subring $A_{n}$ generated by $X_{\alpha}$, with $\alpha \in 2^{-n} Z_{2}^{2 g}$ is just the subring generated by

$$
\left\{Y_{U} \mid U=U+2^{n} Z_{2}^{g}, U \subset 2^{-n} Z_{2}^{g}\right\}
$$

or by

$$
\left\{Y_{\alpha+2^{n} Z_{2}^{g}} \mid \alpha \in 2^{-n} Z_{2}^{g}\right\} \text {. }
$$

The group $G=\operatorname{Sp}\left(2 g, Q_{2}\right)$ acts on $\bar{M}_{\infty}$ in the following way:

Definition 5. For all $\sigma \in G$, let

(*) $U_{\sigma}\left(X_{\alpha}\right)=\sum_{\beta \in Z_{2}^{2} g / Z_{2}^{2} g \cap \sigma^{-1}\left(Z_{2}^{2} z\right)} e_{*}(\beta / 2) e(\beta / 2, \alpha) \cdot e(\gamma / 2, \alpha-\beta) \cdot X_{\sigma \alpha-\sigma \beta-\sigma \gamma}$, where $\gamma \in Q_{2}^{2}$ g is some fixed element satisfying

$$
e_{*}(\beta / 2) \cdot e_{*}(\sigma \beta / 2)=e(\gamma, \beta)
$$

for all $\beta \in Z_{2}^{2 g} \cap \sigma^{-1}\left(Z_{2}^{2 g}\right)$.

Concerning this definition, one verifies by mechanical calculation the following:

1. If the $\gamma$ in the definition is varied, it must change by an element of $Z_{2}^{2 g}+\sigma^{-1}\left(Z_{2}^{2 g}\right)$, and, if so, the operator $U_{\sigma}$ is only changed into a constant multiple of itself :

$$
U_{\sigma}^{\prime}\left(X_{\alpha}\right)=c \cdot U_{\sigma}\left(X_{\alpha}\right), \quad \text { all } \alpha .
$$

We shall assume that for each $\sigma$, some fixed $\gamma$ is chosen.

Note that $-I \in \operatorname{Sp}\left(2 g, Q_{2}\right)=G$, and $U_{-I}\left(X_{\alpha}\right)=X_{-\alpha}$.

2. For all $\sigma \in G, \alpha \in Q_{2}^{2} \mathrm{~g}$ and $\beta \in Z_{2}^{2} \mathrm{~g}$,

$$
U_{\sigma}\left(X_{\alpha+\beta}\right)=e_{*}(\beta / 2) e(\beta / 2, \alpha) \cdot U_{\sigma}\left(X_{\alpha}\right) \text {. }
$$

Therefore, if we let $M$ be the free $R$-module spanned by the $X_{\alpha}$ 's modulo only relations (1.), each $U_{\sigma}$ defines an $R$-module homomorphism from $M$ to $M$.

3. For all $\sigma, \tau \in G$, there is a non-zero constant $c_{\sigma, \tau}$ such that

$$
U_{\sigma} \circ U_{\tau}=c_{\sigma, \tau} \cdot U_{\sigma \cdot \tau},
$$

i. e., $\sigma \mapsto U_{\sigma}$ is a "projective" representation of $G$ in $M$.

4. For all $\sigma \in G$, one computes easily that there is a sign $e_{\sigma}= \pm 1$ such that $U_{-I} \circ U_{\sigma}=e_{\sigma} U_{\sigma} \circ U_{-I}$. Unfortunately, it does not appear to be easy to show directly that $e_{\sigma}=+1$ for all $\sigma$. For example, if $\sigma\left(Z_{2}^{2 g}\right)=Z_{2}^{2 g}$, $e_{\sigma}=e_{*}(\gamma)$. However, using (3.), one checks that

$$
\varepsilon_{\sigma \tau}=\varepsilon_{\sigma} \cdot \varepsilon_{\tau}
$$

and since $G$ is well known to be its own commutator subgroup, this implies $\varepsilon_{\sigma}=+1$, all $\sigma$. But now the submodule $A_{1}$ of $A$ of elements of degree 1 is just $M$ modulo the span of the elements $\left\{X_{\alpha}-X_{-\alpha}\right\}$, and this 


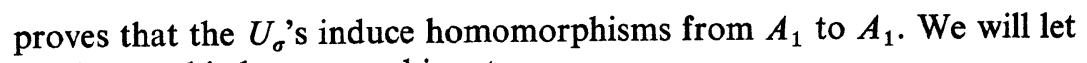
$U_{\sigma}$ denote this homomorphism too.

5. The last step is to check that all the $U_{\sigma}$ 's induce ring homomorphisms from $A$ to $A$. Frankly, $I$ balked at directly applying $U_{\sigma}$ to the relations (3.) and seeing what comes out. But, in 2 special cases, it isn't too bad. Suppose first that $\sigma$ is in the subgroup $\Gamma=\operatorname{Sp}\left(2 g, Z_{2}\right)$. Then $\sigma\left(Z_{2}^{2 g}\right)=Z_{2}^{2 g}$, and $U_{\sigma}$ reduces to:

$$
U_{\sigma}\left(X_{\alpha}\right)=e(\gamma / 2, \alpha) \cdot X_{\sigma \alpha-\sigma \gamma} .
$$

In this case, it's not hard to check that $U_{\sigma}$ takes a relation of type (3.) to another relation of the same type, so that $U_{\sigma}$ induces a map from $A$ to $A$. Suppose second that $\sigma$ is in the sub-semi-group:

$$
H^{+}=\left\{\left(\frac{A \mid 0}{\left.0\right|^{t} A^{-1}}\right) \mid \begin{array}{l}
A \in G L\left(g, Q_{2}\right) \\
A\left(Z_{2}^{\mathrm{g}}\right) \supseteq Z_{2}^{\mathrm{g}}
\end{array}\right\} \subset G .
$$

For such $\sigma, U_{\sigma}$ reduces to:

$$
U_{\sigma}\left(X_{\alpha}\right)=\sum_{\beta \in Z_{2}^{z / A} / A^{-1}} e\left(-\beta_{1}^{\beta} / 2, \alpha\right) \cdot X_{\sigma \alpha+\sigma \beta_{1}}
$$

(where $\beta_{1}$ is the $2 g$-vector $(\beta, 0)$ ). Now, referring back to the proof of Lemma 2 , in the last section, we see that an equivalent form of the relations (3.) is:

where

$$
Y_{\alpha, \beta} \cdot Y_{\gamma, \delta}=Y_{\alpha, \delta} \cdot Y_{\gamma, \beta}, \quad \text { all } \alpha, \beta, \gamma, \delta \in Q_{2}^{2 g}
$$

$$
Y_{\alpha, \beta}=\sum_{\eta \in \frac{1}{2} Z Z_{\frac{z}{2} / Z_{2}}} e\left(-\eta_{1}, \alpha\right) X_{\alpha+\beta+\eta_{1}} \cdot X_{\alpha-\beta+\eta_{1}} .
$$

For $\sigma \in H^{+}, Y_{\alpha, \beta}$ behaves very nicely. One computes easily that:

$$
U_{\sigma}\left(Y_{\alpha, \beta}\right)=\sum_{\eta, \zeta \in \frac{1}{2} A Z_{\frac{z}{2} / \frac{1}{2}} \frac{\beta}{2}} e\left(-\eta_{1}, \sigma \alpha\right) \cdot e\left(-\zeta_{1}, \sigma \beta\right) \cdot Y_{\sigma \alpha+\eta_{1}, \sigma \beta+\zeta_{1}} .
$$

From this it follows immediately that $U_{\sigma}\left[Y_{\alpha, \beta} \cdot Y_{\gamma, \delta}-Y_{\alpha, \delta} \cdot Y_{\gamma, \beta}\right]$ is an $R$-linear combination of expressions $Y_{\alpha^{\prime}, \beta^{\prime}} \cdot Y_{\gamma^{\prime}, \delta^{\prime}}-Y_{\alpha^{\prime}, \delta^{\prime}} \cdot Y_{\gamma^{\prime}, \beta^{\prime}}$.

Finally, it follows from the paper of IWAHORI-MATSUMOTO [13] that all the double cosets in $\Gamma \mid G / \Gamma$ are represented by matrices:

$$
\begin{gathered}
\sigma=\left(\begin{array}{c|c}
A & 0 \\
\hline 0 & t^{t} A^{-1}
\end{array}\right) \\
A=\left(\begin{array}{ccc}
2^{a_{1}} & & \\
& 2^{a_{2}} & \\
& \ddots & \\
0 & 2^{a_{g}}
\end{array}\right) \\
0 \geqq a_{1} \geqq a_{2} \geqq \cdots \geqq a_{g} .
\end{gathered}
$$


Since there are in $H^{+}, G=\Gamma \cdot H^{+} \cdot \Gamma$ and our 2 calculations suffice to prove that for every $\sigma \in G, U_{\sigma}$ maps $A$ to $A$.

Putting all this together, we conclude that Definition 5 defines a projective representation of $G$ on $A$, and an action of $G$ on the scheme $\bar{M}_{\infty}$. In fact, let $V_{\sigma}: \bar{M}_{\infty} \rightarrow \bar{M}_{\infty}$ denote this action, then, by definition,

$$
V_{\sigma}^{*}\left(X_{\alpha}\right)=U_{\sigma^{-1}}\left(X_{\alpha}\right)
$$

(the $\sigma^{-1}$ makes it an action of $G$ instead of the opposed group).

Now we can connect $\bar{M}_{\infty}$ to the moduli problem. For all algebraically closed fields $k(\operatorname{char}(k) \neq 2)$, there is a map:

$$
\Theta: \mathscr{M}_{\infty}(k) \rightarrow\left\{\begin{array}{l}
\text { Set of } k \text {-valued } \\
\text { points of } \bar{M}_{\infty}
\end{array}\right\}
$$

( $\mathscr{M}_{\infty}$ denotes the set defined at the beginning of this $\S$ ), which assigns to a tower $\mathscr{T}$ and a $\varphi: V\left(\underset{X)}{\stackrel{\approx}{\rightarrow}} Q_{2}^{2 \mathrm{~g}}\right.$, the point with homogeneous coordinates

$$
X_{\alpha}=\vartheta\left[\begin{array}{l}
0 \\
0
\end{array}\right]\left(\varphi^{-1} \alpha\right), \quad \text { or } \quad Y_{U}=\mu(U)
$$

(cf. Lemma 1 and $2, \S 8$ ).

Recall that $G$ acts on $\mathscr{M}_{\infty}(k)$ as follows:

Definition 6. Let $(\mathscr{T}, \varphi) \in \mathscr{M}_{\infty}(k)$ and $\sigma \in G$. Then let $U_{\sigma}((\mathscr{T}, \varphi))$ be the pair $(\mathscr{T}, \sigma \circ \varphi)$, i.e., modify the symplectic isomorphism $\sigma$ to:

$$
V\left(\underset{\leftarrow}{X} \underset{\varphi}{\underset{\sim}{\approx}} Q_{2}^{2 g} \underset{\sigma}{\underset{\sim}{\approx}} Q_{2}^{2 g} .\right.
$$

Theorem 1. Under $\Theta$, the actions of $G$ on $\mathscr{M}_{\infty}(k)$ and on the set of $k$-valued points of $\bar{M}_{\infty}$ are compatible. Proof. Recall that $\vartheta\left[\begin{array}{l}0 \\ 0\end{array}\right]$ is the unique element $f$ of $\vartheta(\Gamma(\mathscr{T}))$ in-
variant satisfying

a)

$$
f(x+a)=e_{*}(a / 2) \cdot e_{\lambda}(a / 2, x) \cdot f(x)
$$

for all $a \in \varphi^{-1}\left(Z_{2}^{2 g}\right)$, with $e_{*}\left(\varphi^{-1}(\alpha)\right)=\chi\left(2^{t} \alpha_{1} \cdot \alpha_{2}\right)$, all $\alpha \in \frac{1}{2} Z_{2}^{2 g}$. If we use the symplectic isomorphism $\sigma \circ \varphi$, the new function $\vartheta\left[\begin{array}{l}0 \\ 0\end{array}\right]$ will instead
be the unique $f$ in $\vartheta(\Gamma(\mathscr{T})$ ) satisfying:

$\left.\mathbf{a}^{\prime}\right)$

$$
f(x+a)=e_{*}^{\prime}(a / 2) \cdot e_{\lambda}(a / 2, x) \cdot f(x)
$$

for all $a \in \varphi^{-1}\left(\sigma^{-1}\left(Z_{2}^{2 g}\right)\right)$, with $e_{*}^{\prime}\left(\varphi^{-1}\left(\sigma^{-1}(\alpha)\right)\right)=\chi\left(2^{t} \alpha_{1} \cdot \alpha_{2}\right)$ all $\alpha \in \frac{1}{2} Z_{2}^{2 g}$. Let $\vartheta^{\sigma}\left[\begin{array}{l}0 \\ 0\end{array}\right]$ denote this new $\vartheta\left[\begin{array}{l}0 \\ 0\end{array}\right]$. Then

$$
X_{\alpha}\left[U_{\sigma}(\mathscr{T}, \varphi)\right]=\vartheta^{\sigma}\left[\begin{array}{l}
0 \\
0
\end{array}\right]\left(\varphi^{-1}(\alpha)\right)
$$


To find $\vartheta^{\sigma}\left[\begin{array}{l}0 \\ 0\end{array}\right]$, recall that the functions

$$
\alpha \mapsto e_{\lambda}(\beta / 2, \alpha) \cdot \vartheta\left[\begin{array}{l}
0 \\
0
\end{array}\right](\alpha-\beta), \quad \beta \in V(\underset{\leftarrow}{X}) .
$$

span $V(\underset{\leftarrow}{X})$. Therefore, it suffices to find some linear combination of these functions satisfying $\left.\mathrm{a}^{\prime}\right)$. If we make all these into functions on $Q_{2}^{2 g}$ via $\sigma \circ \varphi: V\left(\underset{X}{X} \stackrel{\sim}{\rightarrow} Q^{2 g}\right.$, we find that

$$
\vartheta^{\sigma}\left[\begin{array}{l}
0 \\
0
\end{array}\right]\left(\varphi^{-1}\left(\sigma^{-1}(\alpha)\right)\right)
$$

is that linear combination $f$ of the functions

such that

$$
\begin{aligned}
g_{\beta}(\alpha) & =e(\beta / 2, \alpha) \cdot g\left(\sigma^{-1} \alpha-\sigma^{-1} \beta\right), \quad \beta \in Q_{2}^{2 g} \\
g(\alpha) & =\vartheta\left[\begin{array}{l}
0 \\
0
\end{array}\right]\left(\varphi^{-1} \alpha\right)
\end{aligned}
$$

$$
\left.\mathrm{a}^{\prime \prime}\right) \quad f(\alpha+\beta)=e_{*}(\beta / 2) \cdot e(\beta / 2, \alpha) \cdot f(\alpha),
$$

for all $\beta \in Z_{2}^{2 g}$. One solution of $\left(\mathrm{a}^{\prime \prime}\right)$ is the function:

$$
\begin{aligned}
g^{\sigma}(\alpha)= & \sum_{\beta \in Z_{2}^{2} g / \mathbf{Z}_{2}^{2} g \cap \sigma \mathbf{Z}_{2}^{2} \mathbf{g}} e_{*}(\beta / 2) \cdot e(\beta / 2, \alpha) \cdot e(\gamma / 2, \alpha-\beta) \\
& \times g\left(\sigma^{-1} \alpha-\sigma^{-1} \beta-\sigma^{-1} \gamma\right)
\end{aligned}
$$

where $\gamma$ satisfies $e_{*}(\beta / 2) \cdot e_{*}\left(\sigma^{-1} \beta / 2\right)=e(\gamma, \beta)$, all $\beta \in Z_{2}^{2 g} \cap \sigma Z_{2}^{2 g}$. If this function is not 0 , it must equal

$$
\vartheta^{\sigma}\left[\begin{array}{l}
0 \\
0
\end{array}\right] \circ \varphi^{-1} \circ \sigma^{-1}
$$

But on the other hand, $X_{\alpha}$ has values $g(\alpha)$ at the geometric point $x$ corresponding to $(\mathscr{T}, \varphi)$. Hence $X_{\alpha}$ has the values at $V_{\sigma}(x)$ :

$$
\begin{aligned}
X_{\alpha}\left(V_{\sigma}(x)\right)= & V_{\sigma}^{*} X_{\alpha}(x) \\
= & U_{\sigma-1} X_{\alpha}(x) \\
= & \sum_{\beta \in Z_{\frac{2}{2}} g / Z_{2}^{\frac{2}{2}} \mathrm{~g} \cap \sigma \mathbf{Z}_{\frac{2}{2}} \mathrm{~g}} e_{*}(\beta / 2) \cdot e(\beta / 2, \alpha) \times \\
& \times e(\gamma / 2, \alpha-\beta) X_{\sigma-1} \alpha-\sigma^{-1 \beta-\sigma^{-1} \gamma}(x) \\
= & g^{\sigma}(\alpha) .
\end{aligned}
$$

Therefore, $g^{\sigma} \neq 0$, so $g^{\sigma}(\alpha)$ is also the value of $X_{\alpha}$ at the geometric point corresponding to $(\mathscr{T}, \sigma \circ \varphi)$. Q.E.D. 
Now every moduli space is supposed to represent a functor. In our case, instead of making a big fuss over defining a family of 2-towers of abelian varieties, over a scheme $S$, it is simpler to observe that $\mathscr{M}_{\infty}(k)$ is an inverse limit of some of the $\mathscr{M}_{\delta}(k)$ 's introduced in $\S 6$, and then to define $\mathscr{M}_{\infty}(S)$ as the corresponding limit of these $\mathscr{M}_{\delta}(S)$ 's.

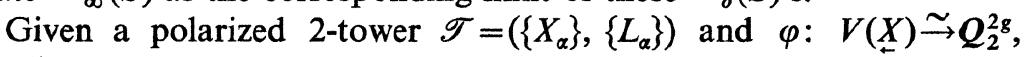
for all $n \geqq 1$, we get

a) an abelian variety $X_{n}=X_{\alpha_{n}}$, where

$$
T\left(\alpha_{n}\right)=\varphi^{-1}\left(2^{-n} Z_{2}^{2 g}\right),
$$

b) a totally symmetric ample invertible sheaf

$$
\begin{aligned}
& L_{n}=L_{\alpha_{n}} \text { on } X_{n}, \text { of type } \\
& \delta_{n}=\left(2^{2 n}, 2^{2 n}, \ldots, 2^{2 n}\right),
\end{aligned}
$$

c) a symmetric $\vartheta$-structure:

This is a map

$$
\lambda_{n}: \mathscr{G}\left(L_{n}\right) \cong \frac{\mathscr{G}_{\alpha_{n}}^{*}(\mathscr{T})}{K\left(\alpha_{n}\right)} \underset{\text { via } \varphi}{\cong} \frac{k^{*} \times 2^{-n} Z_{2}^{2 g}}{\{1\} \times 2^{n} Z_{2}^{2 g}} \cong \mathscr{G}\left(\delta_{n}\right) .
$$

$$
\begin{aligned}
& (\mathscr{T}, \varphi) \mapsto\left(X_{n}, L_{n}, \lambda_{n}\right) \\
& \mathscr{M}_{\infty}(k) \rightarrow \mathscr{M}_{\delta_{n}}(k) .
\end{aligned}
$$

Note that all these $X_{n}$ 's are canonically isomorphic, e.g., to $X_{1}$, via diagrams:

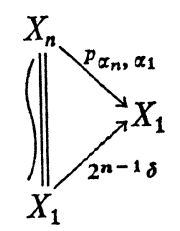

and that under these isomorphisms, $L_{n}$ is just $L_{1}^{4 n-1}$.

On the other hand, between the functors $\mathscr{M}_{\delta_{n+1}}$ and $\mathscr{M}_{\delta_{n}}$, we have a natural transformation:

$$
\pi_{n}: M_{\delta_{n+1}} \rightarrow M_{\delta_{n}} .
$$

In fact, given $\mathscr{X} / S, L$, and $\lambda$ in $\mathscr{M}_{\delta_{n+1}}(S)$, we get

a) a second ample sheaf $M$ on $\mathscr{X}$, by descending $L$ with respect to the isogeny

$2 \delta: \mathscr{X} \rightarrow \mathscr{X}$ 
and the descent data $\lambda^{-1}\left(K_{2}\right)$, where $K_{2} \subset \mathscr{G}\left(\delta_{n+1}\right) \times S$ is the subscheme representing the subfunctor of triples $(1, x, l), 2 x=2 l=0$.

b) Since

$$
\begin{aligned}
\mathscr{G}(M) & \cong \frac{\text { normalizer of } \lambda^{-1}\left(K_{2}\right) \text { in } \mathscr{G}(L)}{\lambda^{-1}\left(K_{2}\right)} \\
& \cong \frac{\text { normalizer of } K_{2} \text { in } \mathscr{G}\left(\delta_{n+1}\right)}{K_{2}} \cong \mathscr{G}\left(\delta_{n}\right)
\end{aligned}
$$

we get a $\vartheta$-structure $\mu: \mathscr{G}(M) \stackrel{\approx}{\rightarrow} \mathscr{G}\left(\delta_{n}\right)$.

It can be checked that $(\mathscr{X} / S, M, \mu) \in \mathscr{G}_{\delta_{n}}(S)$, so we call this $\pi_{n}((\mathscr{X} / S$, $L, \lambda)$ ).

Going back to $k$-valued points, we have a diagram:

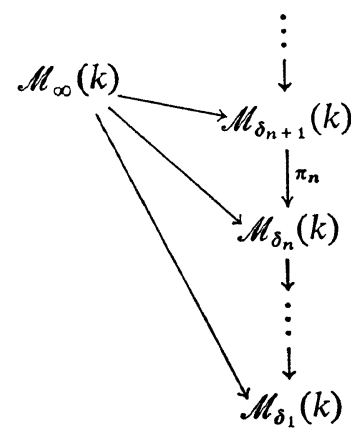

and it is clear that this induces an isomorphism

$$
\mathscr{M}_{\infty}(k) \cong \varliminf \mathscr{M}_{\delta_{n}}(k) \text {. }
$$

Definition 7. For all schemes $S$, let $\mathscr{M}_{\infty}(S)=\varliminf_{\delta_{n}} \mathscr{M}_{\delta_{n}}(S)$.

Next, let's translate the results of $\S 6$ on the representability of $\mathscr{M}_{\delta_{n}}$ into the discussion. The results there show that there is an open set:

$$
\begin{gathered}
M_{\delta_{n}} \subset \operatorname{Proj}\left(A_{n}^{*}\right)=\bar{M}_{\delta_{n}} \\
A_{n}^{*}=R\left[\ldots, Q^{(n)}(a), \ldots\right] /\left\{\begin{array}{l}
\text { Modulo } Q^{(n)}(a)=Q^{(n)}(-a) \\
\text { and certain quartic relations }
\end{array}\right\} a \in K\left(\delta_{n}\right)
\end{gathered}
$$

such that $M_{\delta_{n}}$ represented $\mathscr{M}_{\delta_{n}}$ (over the ring $R$ ). Moreover, in Step II of the proof, a canonical morphism

$$
\pi: \bar{M}_{2 \delta} \rightarrow \bar{M}_{\delta}
$$


was introduced. Iterated, this defines a morphism from $\bar{M}_{4 \delta} \rightarrow \bar{M}_{\delta}$, e.g., from $\bar{M}_{\delta_{n+1}}$ to $\bar{M}_{\delta_{n}}$. If you work it out, it is just the projection:

$$
\begin{gathered}
\bar{M}_{\delta_{n+1}} \stackrel{\pi_{n}}{\longrightarrow} \bar{M}_{\delta_{n}} \\
\pi_{n}^{*}\left(Q^{(n)}(a)\right)=\sum_{\substack{2 b=0 \\
b \in K\left(\delta_{n+1}\right)}} Q^{(n+1)}(b), \quad \text { all } a \in K\left(\delta_{n}\right)
\end{gathered}
$$

(here we identify $K\left(\delta_{n}\right)$ with a subgroup of $K\left(\delta_{n+1}\right)$ as before). In particular, $\pi_{n}$ is a finite morphism. Moreover, as we saw in $\S 6$, (Step II).

(a) $\pi_{n}$ is étale at all points of $M_{\delta_{n+1}}$ and $\pi_{n}^{-1}\left(M_{\delta_{n}}\right)=M_{\delta_{n+1}}$. In fact:

(b) The morphism of schemes $\pi_{n}: M_{\delta_{n+1}} \rightarrow M_{\delta_{n}}$ corresponds to the transformation of functors $\pi_{n}: \mathscr{M}_{\delta_{n+1}} \rightarrow \mathscr{M}_{\delta_{n}}$ via the representability proven in $\S 6$.

(This is easy to check and we omit the proof.)

Now, passing to the limit over the homomorphisms $\pi_{n}^{*}$, note that the direct limit of the rings $A_{n}^{*}$ is just $A$ itself. In fact,

and let

$$
K\left(\delta_{n}\right)=\underset{g \text { times }}{\oplus} Z / 2^{2 n} Z,
$$

$$
\left.Q^{(n)}\left(a_{1}, \ldots, a_{g}\right) \mapsto Y_{(2-n} a_{1}, \ldots, 2-n a_{n}\right)+2^{n} Z_{2}^{g}
$$

$\left(a_{i} \in Z / 2^{2 n} Z\right)$. It is easy to check that the relations imposed on the $Q^{(n)}(a)$ 's give us exactly the defining relations on the $Y_{U}$ 's. Moreover, $A_{n}$ is just the image of $A_{n}^{*}$ in $A$. (It may very well happen that each $\pi_{n}^{*}$ is injective, so that $A \cong \bigcup A_{n}^{*}$ and $A_{n} \cong A_{n}^{*}$ : but $I$ have no proof for this.) Geometrically, this shows that

$$
\bar{M}_{\infty} \cong \varliminf_{n} \bar{M}_{\delta_{n}} .
$$

Definition 8. Let $M_{\infty}$ be the inverse image in $\bar{M}_{\infty}$ of $M_{\delta_{n}}$ in $\bar{M}_{\delta_{n}}$ (independent of $n$ by (a)).

1. $M_{\infty} \cong \varliminf_{\delta_{n}}$

2. $\mathscr{M}_{\infty}(S) \cong \varliminf \mathscr{M}_{\delta_{n}}(S)$

3. The $M_{\delta_{n}}$ 's represent $\mathscr{M}_{\delta_{n}}$ (compatibly as $n$ varies). Hence:

Theorem 2. The scheme $M_{\infty}$ represents $\mathscr{M}_{\infty}$. 
Recapitulating the discussion, the basic set which we are classifying is:

$\mathscr{M}_{\infty}(k)=\left\{\begin{array}{l}\text { set of polarized 2-towers } \mathscr{T}=\left(X_{\alpha}, L_{\alpha}\right) \\ \text { plus symplectic isomorphisms } \varphi: V(\mathscr{T}) \stackrel{\sim}{\longrightarrow} Q_{2}^{2 g}\end{array}\right\}$

$$
=\left\{\begin{array}{l}
\text { set of abelian varieties } X \text {, totally } \\
\text { symmetric ample } L \text { on } X \text { of type }(4,4, \ldots, 4), \\
\text { and symmetric } \vartheta \text {-structures } \lambda_{n} \text { on } \mathscr{G}\left(\left(2^{n} \delta\right)^{*} L\right), \\
\text { for all } n, \text { which are "compatible" as } n \text { varies }
\end{array}\right\} \text {. }
$$

Quite clearly, we can also say:

$\mathscr{M}_{\infty}(k)=\left\{\begin{array}{l}\text { set of abelian varieties } X, \text { totally symmetric } \\ \text { ample } L \text { on } X \text { of type }(4,4, \ldots, 4), \text { and symplectic } \\ \text { isomorphisms } \varphi: V(X) \stackrel{\approx}{\longrightarrow} Q_{2}^{2 g} \text { such that } \varphi(T(X))=2 Z_{2}^{2 g}\end{array}\right\}$, or:

$\mathscr{M}_{\infty}(k)=\left\{\begin{array}{l}\text { set of abelian varieties } X \text {, symmetric ample } L \text { on } X \text { of } \\ \text { degree } 1, \text { and symplectic isomorphisms: } \varphi: V(X) \longrightarrow Q_{2}^{2 g} \\ \text { such that } \varphi(T(X))=Z_{2}^{2 g} \text {, and } e_{*}^{L}\left(\varphi^{-1}(\alpha)\right)=\chi\left(2^{t} \alpha_{1} \cdot \alpha_{2}\right) \text { all } \\ \alpha \in \frac{1}{2} Z_{2}^{2 g}\end{array}\right\}$.

In any case, the principal result, on the level of geometric points, is:

Corollary. For all algebraically closed fields $k$, the map $\Theta$ is a bijection between $\mathscr{M}_{\infty}(k)$ and the set of $k$-valued points of the open set $M_{\infty}$.

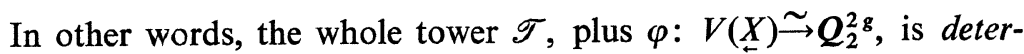
mined by the theta function

$$
\vartheta\left[\begin{array}{l}
0 \\
0
\end{array}\right] \circ \varphi^{-1}
$$

(or the measure $\mu$ ) and the theta functions that arise in this way are those which satisfy some finite set of inequalities. Our next task is to determine these inequalities, and hence $M_{\infty}$, explicitly.

Department of Mathematics Harvard University Cambridge, Massachussetts 